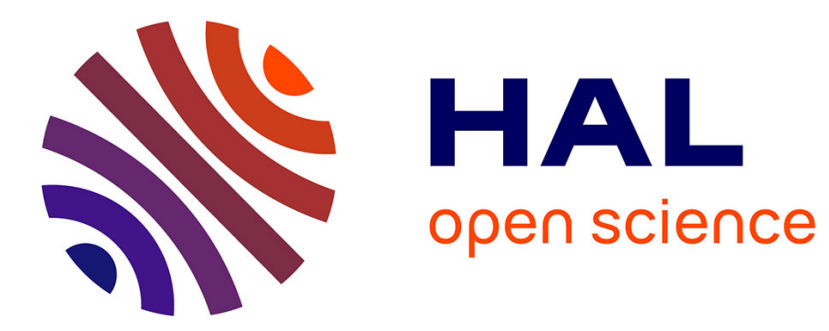

\title{
A center manifold result for delayed neural fields equations
}

\author{
Romain Veltz, Olivier Faugeras
}

\section{To cite this version:}

Romain Veltz, Olivier Faugeras. A center manifold result for delayed neural fields equations. [Research Report] RR-8020, INRIA. 2012. hal-00719794v2

\section{HAL Id: hal-00719794 \\ https://hal.inria.fr/hal-00719794v2}

Submitted on 23 Jan 2013

HAL is a multi-disciplinary open access archive for the deposit and dissemination of scientific research documents, whether they are published or not. The documents may come from teaching and research institutions in France or abroad, or from public or private research centers.
L'archive ouverte pluridisciplinaire HAL, est destinée au dépôt et à la diffusion de documents scientifiques de niveau recherche, publiés ou non, émanant des établissements d'enseignement et de recherche français ou étrangers, des laboratoires publics ou privés. 


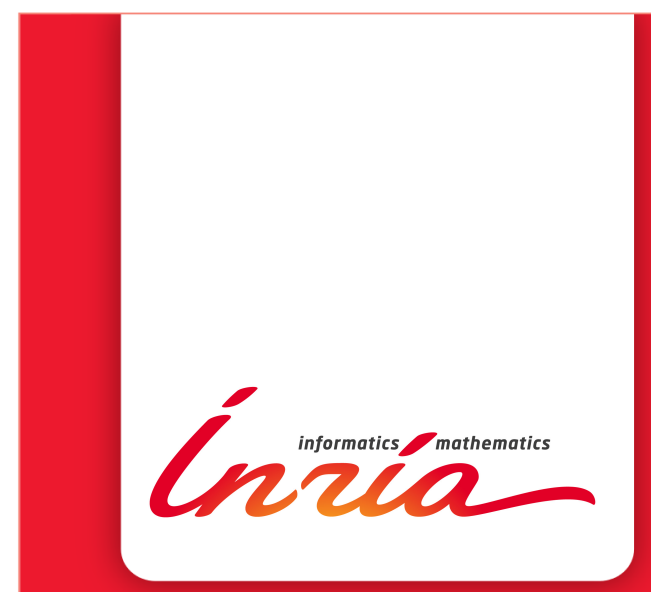

\section{A center manifold result for delayed neural fields equations}

Romain Veltz, Olivier Faugeras

\section{RESEARCH}

\section{REPORT}

$\mathrm{N}^{\circ} 8020$

Juillet 2012

Project-Team NeuroMathComp 



\title{
$\ln 2$
}

\section{A center manifold result for delayed neural fields equations}

\author{
Romain Veltz*, Olivier Faugeras ${ }^{\dagger}$ \\ Project-Team NeuroMathComp
}

Research Report $n^{\circ} 8020$ - Juillet 2012 - 41 pages

\begin{abstract}
We develop a framework for the study of delayed neural fields equations and prove a center manifold theorem for these equations. Specific properties of delayed neural fields equations make it impossible to apply existing methods from the literature concerning center manifold results for functional differential equations. Our approach for the proof of the center manifold theorem uses the original combination of results from Vanderbauwhede etal. together with a theory of linear functional differential equations in a history space larger than the commonly used set of time-continuous functions.
\end{abstract}

Key-words: Neural fields equations, space-dependent delays, bifurcation theory, center manifold, functional differential equations.

This research was partially supported by the European Union Seventh Framework Programme (FP7/20072013) under grant agreement no. 269921 (BrainScaleS) and by the ERC advanced grant NerVi no. 227747.

* IMAGINE/LIGM, Université Paris Est and NeuroMathComp team.

$\dagger$ Corresponding author, NeuroMathComp team, INRIA, CNRS, ENS Paris, romain.veltz@sophia.inria.fr

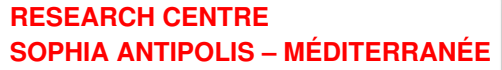




\section{Un théorème de variété centrale pour les équations de masses neurales avec délais.}

Résumé : Dans ce document, nous developpons un cadre théorique pour les équations de masses neurales avec délais et nous prouvons un théorème de variété centrale pour ces équations. Des propriétés spécifiques des équations de masses neurales avec délais rendent difficiles l'application de méthodes existantes issues de la littérature au sujet des variétés centrales pour les équations différentielles fonctionnelles. Nous utilisons, pour la preuve d'un théorème de variété centrale, les résultats de Vanderbauwhede etal. ainsi qu'une théorie des équations différentielles dans un espace des phases plus large que celui des fonctions continues en temps qui est généralement utilisé.

Mots-clés : Equations des masses neurales, délais dépendant de l'espace, théorie des bifurcations, variété centrale, équations différentielles fonctionnelles. 


\section{Introduction}

We consider the autonomous nonlinear functional equation of retarded type:

$$
\dot{\mathbf{V}}(t)=-\mathbf{L}_{0} \mathbf{V}(t)+\mathbf{L}_{1} \mathbf{S}\left(\mathbf{V}_{t}\right)+\mathbf{I}_{e x t}, \quad \mathbf{V}(t), \mathbf{I}_{e x t} \in \mathrm{L}^{2}\left(\Omega, \mathbb{R}^{\mathrm{p}}\right)
$$

where $\Omega$ is an open bounded set of $\mathbb{R}^{d}$ and $\mathbf{L}_{0}=\operatorname{diag}\left(l_{1}, \cdots, l_{p}\right), l_{i}>0$. The nonlinearity $\mathbf{S}: \mathbb{R}^{p} \rightarrow \mathbb{R}_{+}^{p}$ is smooth and bounded and the maximum delay appearing in 1 is written $\tau_{m}$. The linear operator $\mathbf{L}_{1}$ is defined below in (5).

These equations are called delayed neural fields equations and are becoming increasingly important for the neuroscience community (see [30, 38, 3, 4, 13, 10, 27, 28, 7, 39]). For example, the neural fields equations have been used to model the rat barrel cortex [36] and the visual cortex [6,33]. The computation of the stationary states of 1 and their stability is now well documented $[19,8,9,14,23,44]$ and an immediate question is how this stability is altered when delays are introduced. Delays in neural fields equations can be of two types. There are the effective delays and the propagation delays. Propagation delays come the finite propagation speed of signals in neural tissues and effective delays are constant delays introduced to model sophisticated phenomena such as the signal integration between two neurons (see for example $[38,39])$.

The aim of the present work is to prove a center manifold theorem for equations of type 1 in order to apply a normal form theory as exposed in [25]. These center manifold results are particularly interesting for delayed differential equations as they allow a finite dimensional description of the flow on an invariant manifold. Proving center manifold results for neural fields equations is trivial because they are ordinary differential equations in the state space $\mathbf{L}^{2}\left(\Omega, \mathbb{R}^{p}\right)$.

There is a large literature on the center manifold theorem which dates back at least to [37]. We only mention the overview of [41] and also the work in [42] which gives general requirements for the existence of such manifold. These tools have been mainly applied to partial differential equations and we are only aware of [29] for the application of [41] to scalar functional differential equations.

Center manifold results for general functional differential equations in $\mathbb{R}^{n}$ have been proved in [16] where a part of the proof follows the line of the work of Vanderbauwhede etal. while the part concerning the variation-of-constants formula is specific to delays and relies on the sun-dual framework (see for example [11, 18]). The center manifold theorem has also been proved for scalar advance differential equations in [29] using [41] and Fourier distributions. Finally, another proof for functional differential equations of mixed type, i.e. with positive and 'negative' delays have been given in [26]. This last reference makes use of similar tools as in [16].

These center manifold results concern finite dimensional functional differential equations where the righthand side of 1 is in $\mathbb{R}^{n}$. When dealing with delayed partial differential equations, the results presented above do not apply. In [21], Faria etal. proved a center manifold result for equations

$$
\dot{u}(t)=A_{T} u(t)+L\left(u_{t}\right)+F\left(u_{t}\right), \quad u(t) \in X
$$

where $X$ is a Banach space, $r>0, L: C^{0}([-r, 0], X) \rightarrow X$ is a bounded linear operator, $F$ is a smooth function and $A_{T}: D\left(A_{T}\right) \subset X \rightarrow X$ is the infinitesimal generator of a compact strongly continuous semigroup. The hypothesis on $A_{T}$ is motivated by the example of the Laplacian $A_{T}=\Delta$. Note that this hypothesis implies that the spectrum of the infinitesimal generator of the delay semigroup is punctual and that the delay semigroup is eventually compact.

The delayed neural fields equations 1 are not amenable through the work of Faria etal. because the semigroup (which is in fact a group) generated by $-\mathbf{L}_{0}$ is not compact. Therefore we have to prove a center manifold result for 1 . There are two strategies. One would be to modify the 
proof of [21] or [16]. The other is to adapt the history space, i.e. the state space of (1), in order to apply the general result in [41]. We chose the second strategy as it does not require looking at the details of the existence/smoothness of the center manifold function. The main difficulty in the application of [41], and more generally in any analytical proof of center manifold results, is the study of the linear inhomogeneous problem:

$$
\dot{\mathbf{V}}(t)=-\mathbf{L}_{0} \mathbf{V}(t)+\mathbf{L}_{1} \mathbf{V}_{t}+f(t)
$$

where $f$ is in the hyperbolic linear subspace of the delay semigroup of the homogeneous equation. This equation is usually analysed with the variation-of-constant formula and with an exponential estimate of the semigroup on its hyperbolic subspaces. However, this variation-of-constant formula is not well-defined in $C^{0}\left(\left[-\tau_{m}, 0\right], \mathrm{L}^{2}\left(\Omega, \mathbb{R}^{\mathrm{p}}\right)\right)$ (see for example [24]). This is why Diekmann etal. use the sun-dual framework.

Also, for the reduced equation produced by the center manifold theorem, an expression of the spectral projector is required. In the case of the history space $C^{0}([-r, 0], X)$, the spectral projector is computed in [40,2] if $L$ satisfies

$$
L(\phi)=\int_{-r}^{0} d \eta(\theta) \phi(\theta), \quad \phi \in C^{0}([-r, 0], X)
$$

with $\eta:[-r, 0] \rightarrow \mathcal{L}(X, X)$ of bounded variations. We have not been able to put our delay operator $\mathbf{L}_{1}$ into this form. Following [2], we could still compute a spectral projector for more general linear equations than (3) but we find it more convenient to use the space $\mathcal{X}^{(2)}$ as we shall now explain. Another way for analysing (2), explained in [12, 46, 35, 34, 5] and that we will pursue, is to study the homogeneous version of 2 , i.e. with $f=0$, in the larger space $\mathcal{X}^{(2)} \equiv \mathrm{L}^{2}\left(\Omega, \mathbb{R}^{\mathrm{p}}\right) \times \mathrm{L}^{2}\left(-\tau_{\mathrm{m}}, 0 ; \mathrm{L}^{2}\left(\Omega, \mathbb{R}^{\mathrm{p}}\right)\right)$ of $\mathrm{L}^{2}$ time-regular history segments. In this space, the solution of the homogeneous equation define a strongly continuous semigroup on $\mathcal{X}^{(2)}$ which allows to treat the inhomogeneous problem (2) more easily. Indeed, the variation-of-constants formula is well defined for perturbations such as (2) in $\mathcal{X}^{(2)}$. Moreover, $\mathcal{X}^{(2)}$ is a Hilbert space on which spectral projectors are easily computed. Finally, general results [18, 5] concerning the spectral mapping theorem and applied to functional differential equations gives exponential estimates of the semigroup norm. These are all basic ingredients needed for the proof of existence of a center manifold and this justifies the use of $\mathcal{X}^{(2)}$ as the phase space.

Our main goal is to develop and put to good use a normal form theory. This requires to work in a Banach algebra or any Banach space ensuring enough regularity to the righthand side of (1). Our nonlinearity is not smooth enough on $\mathcal{X}^{(2)}$ and we have to restrict the history space to subspaces $\mathcal{X}^{(p)}$ of $\mathcal{X}^{(2)}$ in order to give more regularity to the nonlinearity. Note that our spectral projector in $\mathcal{X}^{(2)}$ still defines a spectral projector in the subspaces $\mathcal{X}^{(p)}$ and thus can be used to decompose the history space $\mathcal{X}^{(p)}$.

The paper is organized as follow. In section 2, some definitions and results are recalled. Section 3 addresses the linear analysis and the computation of the spectral projector. In particular, the spectrum of the infinitesimal generator is characterized as well as the generalized eigenspaces. In section 4, we study the linear inhomogeneous problem (2) and prove the main result of this paper, the center manifold theorem 4.4. As an example, we compute the normal form of the Pitchfork bifurcation. In order for the paper to be relatively self-contained, we recall in appendix A several classical results on operators and their spectra. Appendix B contains the proofs of two technical lemmas which are used in the main text. Appendix $\mathrm{C}$ contains the proofs of three technical lemmas and two propositions that are necessary for the proof of the center manifold theorem 4.4 . 


\section{Preliminaries}

\subsection{Definitions}

We consider the following delayed neural fields equations

$$
\left\{\begin{aligned}
\left(\frac{d}{d t}+l_{i}\right) V_{i}(t, \mathbf{r}) & =\sum_{j=1}^{p} \int_{\Omega} J_{i j}(\mathbf{r}, \overline{\mathbf{r}}) S\left[\sigma_{j} V_{j}\left(t-\tau_{i j}(\mathbf{r}, \overline{\mathbf{r}}), \overline{\mathbf{r}}\right)-h_{j}\right] d \overline{\mathbf{r}} \\
V_{i}(t, \mathbf{r}) \quad & \quad+\quad I_{\text {ext }, i}(\mathbf{r}, t), \quad t \geq 0,1 \leq i, j \leq p \\
& =\phi_{i}(t, \mathbf{r}), \quad t \in\left[-\tau_{m}, 0\right]
\end{aligned}\right.
$$

These equations feature the nonlinear gains $\sigma_{i}>0$ and the thresholds $h_{i}>0$. The function $S: \mathbb{R} \rightarrow[0,1]$ is a smooth, monotonically increasing function, for example $S(x)=1 /\left(1+e^{-x}\right)$.

The functions $\tau_{i j}(\mathbf{r}, \overline{\mathbf{r}})$ are the space dependent delays. They are supposed to be continuous functions to simplify the study and the maximum delay is written:

$$
\tau_{m} \equiv \max _{1 \leq i, j \leq p,(\mathbf{r}, \overline{\mathbf{r}}) \in \bar{\Omega}^{2}} \tau_{i j}(\mathbf{r}, \overline{\mathbf{r}})
$$

In particular applications, the delay terms are often (see $[30,38,3,4,13,10,27,28,7,39]$ ) chosen according to

$$
\tau_{i j}(\mathbf{r}, \overline{\mathbf{r}})=D_{i j}+c_{i j}\|\mathbf{r}-\overline{\mathbf{r}}\|_{2} .
$$

We write $\mathbf{L}_{0}=\operatorname{diag}\left(l_{1}, \cdots, l_{p}\right), l_{i}>0, \mathbf{S}(x)=\left[S\left(\sigma_{1} x_{1}-h_{1}\right), \cdots, S\left(\sigma_{p} x_{p}-h_{p}\right)\right]$ and the delay operator

$$
\left\{\begin{array}{l}
\mathbf{L}_{1}: W^{1,2}\left(-\tau_{m}, 0 ; \mathrm{L}^{2}\left(\Omega, \mathbb{R}^{\mathrm{p}}\right)\right) \longrightarrow \mathrm{L}^{2}\left(\Omega, \mathbb{R}^{\mathrm{p}}\right) \\
\phi \rightarrow \int_{\Omega} \mathbf{J}(\cdot, \overline{\mathbf{r}}) \phi(\overline{\mathbf{r}},-\boldsymbol{\tau}(\cdot, \overline{\mathbf{r}})) d \overline{\mathbf{r}}
\end{array}\right.
$$

where $W^{1,2}\left(-\tau_{m}, 0 ; \mathrm{L}^{2}\left(\Omega, \mathbb{R}^{\mathrm{p}}\right)\right)$ is the Sobolev space of functions with values in $\mathrm{L}^{2}\left(\Omega, \mathbb{R}^{\mathrm{p}}\right)$. This operator is continuous because of lemma B.1. Using these notations, we can write (4) in the compact form

$$
\dot{\mathbf{V}}(t)=-\mathbf{L}_{0} \mathbf{V}(t)+\mathbf{L}_{1} \mathbf{S}\left(\mathbf{V}_{t}\right)+\mathbf{I}_{\text {ext }} .
$$

Finally, we introduce the Banach spaces

$$
\left\{\begin{array}{l}
\mathcal{X}^{(q)} \equiv \mathrm{L}^{\mathrm{q}} \times \mathrm{L}^{\mathrm{q}}\left(-\tau_{\mathrm{m}}, 0 ; \mathrm{L}^{\mathrm{q}}\right) \\
\mathcal{Y}^{(q)} \equiv \mathrm{L}^{\mathrm{q}} \times \mathrm{L}^{\mathrm{q}}\left(-\tau_{\mathrm{m}}, 0 ; \mathrm{L}^{\mathrm{q}}\right) \\
\mathcal{Z}^{(q)} \equiv\left\{u \in \mathrm{L}^{\mathrm{q}} \times \mathrm{W}^{1, \mathrm{q}}\left(-\tau_{\mathrm{m}}, 0 ; \mathrm{L}^{\mathrm{q}}\right) \mid \pi_{1} \mathrm{u}=\left(\pi_{2} \mathrm{u}\right)(0)\right\}
\end{array}\right.
$$

where $\mathrm{L}^{\mathrm{q}} \equiv \mathrm{L}^{\mathrm{q}}\left(\Omega, \mathbb{R}^{\mathrm{p}}\right)$ and

$$
\begin{aligned}
& \|\phi\|_{\mathrm{L}^{\mathrm{q}}\left(-\tau_{\mathrm{m}}, 0 ; \mathrm{L}^{\mathrm{q}}\right)} \quad=\left(\int_{-\tau_{m}}^{0}\|\phi(\theta)\|_{\mathrm{L}^{\mathrm{q}}}^{q} d \theta\right)^{\frac{1}{q}} \\
& \|\phi\|_{\mathrm{W}^{1, \mathrm{q}}\left(-\tau_{\mathrm{m}}, 0 ; \mathrm{L}^{\mathrm{q}}\right)}=\|\phi\|_{\mathrm{L}^{\mathrm{q}}\left(-\tau_{\mathrm{m}}, 0 ; \mathrm{L}^{\mathrm{q}}\right)}+\left\|\frac{d}{d \theta} \phi\right\|_{\mathrm{L}^{\mathrm{q}}\left(-\tau_{\mathrm{m}}, 0 ; \mathrm{L}^{\mathrm{q}}\right)} .
\end{aligned}
$$

Note that the spaces $\mathcal{X}^{(q)}$ are subsets of one another for decreasing $q$ s and that $\mathcal{X}^{(2)}$ is a Hilbert space with inner product:

$$
\left\langle\left[\begin{array}{l}
x \\
\phi
\end{array}\right],\left[\begin{array}{l}
y \\
\psi
\end{array}\right]\right\rangle_{\mathcal{X}^{(2)}}=\langle x, y\rangle_{\mathrm{L}^{2}}+\int_{-\tau_{m}}^{0}\langle\phi(s), \psi(s)\rangle_{\mathrm{L}^{2}} d s .
$$

$\operatorname{RR} \mathrm{n}^{\circ} 8020$ 


\subsection{Results concerning dynamics}

We proved in [45] that the solutions of the problem

$$
\left\{\begin{array}{l}
\dot{\mathbf{V}}(t)=-\mathbf{L}_{0} \mathbf{V}(t)+\mathbf{L}_{1} \mathbf{S}\left(\mathbf{V}_{t}\right)+\mathbf{I}_{e x t}(t) \\
\mathbf{V}_{0}=\phi \in C^{0}\left(-\tau_{m}, 0 ; \mathrm{L}^{2}\right)
\end{array}\right.
$$

exist on the positive real axis and are ultimately bounded if the input $\mathbf{I}_{\text {ext }}$ is bounded.

Proposition 2.1 If the following assumptions are satisfied:

1. $\mathbf{J} \in \mathrm{L}^{2}\left(\Omega^{2}, \mathbb{R}^{\mathrm{p} \times \mathrm{p}}\right)$

2. the external current $\mathbf{I}_{\text {ext }} \in C^{0}\left(\mathbb{R}, \mathrm{L}^{2}\right)$

3. $\tau \in C^{0}\left(\bar{\Omega}^{2}, \mathbb{R}_{+}^{p \times p}\right)$

Then for any initial condition, $\phi \in C^{0}\left(-\tau_{m}, 0 ; \mathrm{L}^{2}\right)$, there exists a unique solution $\mathbf{V} \in C^{1}\left(0, \infty ; \mathrm{L}^{2}\right) \cap$ $\mathrm{C}^{0}\left(-\tau_{\mathrm{m}}, \infty ; \mathrm{L}^{2}\right)$ to $(9)$.

All the trajectories of the equation (9) are ultimately bounded by the same constant $R$ if $\max _{t \in \mathbb{R}^{+}}\left\|\mathbf{I}_{e x t}(t)\right\|_{\mathrm{L}^{2}}<\infty$.

\section{$3 \quad$ Linear analysis}

In the Introduction, we have emphasized the need for the study of the stability of stationary states. Let us consider an equilibrium $\mathbf{V}^{f}$ which has been computed for example with tools from $[19,8,9,14,44]$. How stable to perturbations is this cortical state? This is studied by looking at a perturbation of the form $\mathbf{U}=\mathbf{V}-\mathbf{V}^{f}$ and studying the linearized equation satisfied by $\mathbf{U}$ :

$$
\dot{\mathbf{U}}(t)=-\mathbf{L}_{0} \mathbf{U}(t)+\tilde{\mathbf{L}}_{1} \mathbf{U}_{t} \equiv \mathbf{L U}_{t}
$$

where $\tilde{\mathbf{L}}_{1}$ is given by (a more precise definition is given in (14)):

$$
\tilde{\mathbf{L}}_{1}: \phi \rightarrow \int_{\Omega} \mathbf{J}(\cdot, \overline{\mathbf{r}}) D \mathbf{S}\left(\mathbf{V}^{f}(\overline{\mathbf{r}})\right) \phi(\overline{\mathbf{r}},-\boldsymbol{\tau}(\cdot, \overline{\mathbf{r}})) d \overline{\mathbf{r}} .
$$

Looking at exponential perturbations like $\mathbf{U}(t, \mathbf{r})=e^{\lambda t} \mathbf{U}(\mathbf{r})$ in (10), we find that $\mathbf{U}$ solves the eigenvalue problem

$$
\left(\lambda \mathrm{Id}+\mathbf{L}_{0}\right) \mathbf{U}=\mathbf{J}(\lambda) \mathbf{U}
$$

where $\mathbf{J}(\lambda)$ is the compact operator (being a Hilbert-Schmidt operator)

$$
\left\{\begin{array}{l}
\mathbf{J}(\lambda): \mathrm{L}^{2} \longrightarrow \mathrm{L}^{2} \\
\mathbf{U} \rightarrow \int_{\Omega} \mathbf{J}(\cdot, \overline{\mathbf{r}}) D \mathbf{S}\left(\mathbf{V}^{f}(\overline{\mathbf{r}})\right) e^{-\lambda \boldsymbol{\tau}(\cdot, \overline{\mathbf{r}})} \mathbf{U}(\overline{\mathbf{r}}) d \overline{\mathbf{r}}
\end{array}\right.
$$

\subsection{Semigroup properties from the spectral study}

As we have explained in the introduction, we look at equation (10) in the larger space $\mathcal{X}^{(2)}=$ $\mathrm{L}^{2} \times \mathrm{L}^{2}\left(-\tau_{\mathrm{m}}, 0 ; \mathrm{L}^{2}\right)$ with

$$
\left\langle\left[\begin{array}{l}
x \\
\phi
\end{array}\right],\left[\begin{array}{c}
y \\
\psi
\end{array}\right]\right\rangle_{\mathcal{X}^{(2)}}=\langle x, y\rangle_{\mathrm{L}^{2}}+\int_{-\tau_{m}}^{0}\langle\phi(s), \psi(s)\rangle_{\mathrm{L}^{2}} d s
$$


We note $\pi_{1}, \pi_{2}$ the canonical projections of $\mathcal{X}^{(2)}$ on $\mathrm{L}^{2}$ and $\mathrm{L}^{2}\left(-\tau_{\mathrm{m}}, 0 ; \mathrm{L}^{2}\right): \pi_{1}\left[\begin{array}{l}x \\ \phi\end{array}\right]=x, \quad \pi_{2}\left[\begin{array}{l}x \\ \phi\end{array}\right]=$ $\phi$. Following $[12,46,35,34,5]$, we rewrite the problem (10) in the space $\mathcal{X}^{(2)}$. Note that we need to define two functions for the initial condition of (13) because $\phi(0)$ has no meaning for $\phi \in \mathrm{L}^{2}\left(-\tau_{m}, 0 ; \mathrm{L}^{2}\right)$. Hence we write:

$$
\left\{\begin{array}{l}
\dot{\mathbf{U}}(t)=-\mathbf{L}_{0} \mathbf{U}(t)+\tilde{\mathbf{L}}_{1} \mathbf{U}_{t} \equiv \mathbf{L} \mathbf{U}_{t} \\
\mathbf{U}_{0}=\phi \in \mathrm{L}^{2}\left(-\tau_{\mathrm{m}}, 0 ; \mathrm{L}^{2}\right), \mathbf{U}(0)=\mathrm{x} \in \mathrm{L}^{2}
\end{array}\right.
$$

where the linear operator $\tilde{\mathbf{L}}_{1}$ is given by

$$
\left\{\begin{array}{l}
\tilde{\mathbf{L}}_{1}: W^{1,2}\left(-\tau_{m}, 0 ; \mathrm{L}^{2}\right) \longrightarrow \mathrm{L}^{2} \\
\phi \rightarrow \int_{\Omega} \mathbf{J}(\cdot, \overline{\mathbf{r}}) D \mathbf{S}\left(\mathbf{V}^{f}(\overline{\mathbf{r}})\right) \phi(\overline{\mathbf{r}},-\boldsymbol{\tau}(\cdot, \overline{\mathbf{r}})) d \overline{\mathbf{r}}
\end{array}\right.
$$

This operator is continuous as shown in lemma B.1.

Remark 1 In this section, we have used the space $\mathrm{L}^{2}\left(-\tau_{\mathrm{m}}, 0 ; \mathrm{L}^{2}\right)\left(\right.$ resp. $\left.\mathrm{W}^{1,2}\left(-\tau_{\mathrm{m}}, 0 ; \mathrm{L}^{2}\right)\right)$ of vector valued square integrable functions (resp. vector valued functions of the Sobolev space) without definition. More precisely, the Lebesgue-Bochner (Banach) space $\mathrm{L}^{\mathrm{p}}\left(-\tau_{\mathrm{m}}, 0 ; \mathrm{L}^{2}\right), 1 \leq$ $\mathrm{p}<\infty$ is made of the set of all equivalence classes of measurable functions $\phi:\left[-\tau_{m}, 0\right] \rightarrow \mathrm{L}^{2}$ such that $\|\phi\|_{p} \equiv\left(\int_{-\tau_{m}}^{0}\|\phi(\theta)\|_{L^{2}}^{p} d \theta\right)^{\frac{1}{p}}<\infty$. Being measurable means that $\theta \rightarrow\|\phi(\theta)\|_{L^{2}}$ is integrable and $\int_{-\tau_{m}}^{0}\|\phi(\theta)\|_{\mathrm{L}^{2}} d \theta<\infty$, it is also called Bochner integrable (see for example [49, 17]). The Sobolev space is defined as follow:

$$
\begin{aligned}
\mathrm{W}^{1, \mathrm{p}}\left(-\tau_{\mathrm{m}}, 0 ; \mathrm{L}^{2}\right) \equiv\left\{\phi \in \mathrm{L}^{\mathrm{p}}\left(-\tau_{\mathrm{m}}, 0 ; \mathrm{L}^{2}\right) \mid \exists \psi \in \mathrm{L}^{\mathrm{p}}\left(-\tau_{\mathrm{m}}, 0 ; \mathrm{L}^{2}\right)\right. & \text { such that } \\
& \left.\phi(\theta)=\phi\left(-\tau_{m}\right)+\int_{-\tau_{m}}^{\theta} \psi(s) d s\right\} .
\end{aligned}
$$

Then $\frac{d}{d \theta} \phi=\psi$ for $\phi \in \mathrm{W}^{1, \mathrm{p}}\left(-\tau_{\mathrm{m}}, 0 ; \mathrm{L}^{2}\right)$ and $\|\phi\|_{1, p} \equiv\|\phi\|_{p}+\left\|\frac{d}{d \theta} \phi\right\|_{p}$.

Note that $\mathbf{L}_{0}$ is defined on $\mathrm{L}^{2}\left(-\tau_{\mathrm{m}}, 0 ; \mathrm{L}^{2}\right)$ whereas $\tilde{\mathbf{L}}_{1}$ is defined on $W^{1,2}\left(-\tau_{m}, 0 ; \mathrm{L}^{2}\right)$. We call a classical solution of (13) a function $\mathbf{U} \in C^{0}\left(\left[-\tau_{m}, \infty\right), \mathrm{L}^{2}\right) \cap \mathrm{C}^{1}\left([0, \infty), \mathrm{L}^{2}\right)$ such that $\mathbf{U}_{t} \in W^{1,2}\left(-\tau_{m}, 0 ; \mathrm{L}^{2}\right)$ and $\mathbf{U}$ satisfies (13). To study (13), let us introduce the abstract Cauchy problem

$$
\left\{\begin{array}{l}
\dot{u}=\mathbf{A} u \\
u(0)=u_{0} \in \mathcal{X}^{(2)}
\end{array}\right.
$$

where $\mathbf{A}$ is the operator:

$$
\mathbf{A} \equiv\left[\begin{array}{cc}
-\mathbf{L}_{0} & \tilde{\mathbf{L}}_{1} \\
0 & \frac{d}{d \theta}
\end{array}\right]
$$

with domain

$$
D(\mathbf{A})=\left\{\left[\begin{array}{l}
x \\
\phi
\end{array}\right] \in \mathrm{L}^{2} \times \mathrm{W}^{1,2}\left(-\tau_{\mathrm{m}}, 0 ; \mathrm{L}^{2}\right), \phi(0)=\mathrm{x}\right\} .
$$

$\mathrm{RR} \mathrm{n}^{\circ} 8020$ 
From [5], it is known that (13) and (15) are equivalent: every integral/classical solution of (13) is a mild/strong solution of (15) and conversely. This allows to apply the semigroup theory to (15) in order to study (13). More precisely, following [5][theorem 4.23] (see also [18, 1, 24, 47, 15] in a different functional setting), $\mathbf{A}$ generates a strongly continuous semigroup $(\mathbf{T}(t))_{t>0}$ on $\mathcal{X}^{(2)}$ for which $\mathbf{A}$ is the infinitesimal generator. Every solution of (15) is given by $\mathbf{T}(t) u_{0}$ : if $u_{0} \in D(\mathbf{A})$, then $\mathbf{T}(t) u_{0}$ is a strong solution of (15). Hence, any solution $\mathbf{U}$ of (13) is given by $\mathbf{U}(t)=\pi_{1} \mathbf{T}(t) u_{0}$.

Let us turn to the study of the spectral properties: we want to find an estimate of $\|\mathbf{U}(t)\|_{\mathrm{L}^{2}}$ with the spectrum $\Sigma(\mathbf{A})$ of $\mathbf{A}$, i.e. to link estimates of the semigroup $\mathbf{T}$ to the spectrum of $\mathbf{A}$. This is achieved using the Spectral Mapping Theorem (see [18, 5] and theorem A.4). Recall (see $[48,24,18,5,45])$ that $\lambda$ is in the spectrum $\Sigma(\mathbf{A})$ of $\mathbf{A}$ if and only if the operator

$$
\Delta(\lambda) \equiv \lambda \mathrm{Id}+\mathbf{L}_{0}-\mathbf{J}(\lambda) \in \mathcal{L}\left(\mathrm{L}^{2}, \mathrm{~L}^{2}\right)
$$

is not invertible and that $u$ is an eigenvector of $\mathbf{A}$ if and only if $u(\theta, \mathbf{r})=\left[\begin{array}{c}\mathbf{U}(\mathbf{r}) \\ e^{\lambda \theta} \mathbf{U}(\mathbf{r})\end{array}\right]$ with $\mathbf{U} \in \operatorname{ker} \Delta(\lambda)$. Hence the characteristic values (also written $\mathrm{CV}$, see definition A.10) of $\lambda \rightarrow \Delta(\lambda)$ are the eigenvalues of $\mathbf{A}$. We denote the set of eigenvalues by $\Sigma_{p}(\mathbf{A})$, the point spectrum. In order to characterize the spectrum $\Sigma(\mathbf{A})$, we find it convenient to split it into the essential spectrum $\Sigma_{\text {ess }}(\mathbf{A})$ and the point spectrum. As we intend to use the theory of Kato in [31] to study the spectrum, we chose the definition of Kato of the essential spectrum, i.e.

$$
\Sigma_{\text {ess }}(\mathbf{A}) \equiv\{\lambda \in \mathbb{C} \mid \lambda \mathrm{Id}-\mathbf{A} \text { is not semi-Fredholm }\}
$$

(see definitions A.7 and A.8 for the definition of Fredholm and semi-Fredholm operators). This definition is different from the one adopted in $[18,5]$ which is $\Sigma_{\text {ess }}$ Engel $(\mathbf{A}) \equiv\{\lambda \in \mathbb{C} \mid \lambda \mathrm{Id}-\mathbf{A}$ is not Fredholm $\}$. We state a useful lemma which links the spectral properties of $\mathbf{A}$ to the spectral properties of $-\mathbf{L}_{0}+\mathbf{J}(\lambda) \in \mathcal{L}\left(\mathrm{L}^{2}, \mathrm{~L}^{2}\right)$.

Lemma $3.1 \lambda \in \Sigma_{\text {ess }}(\mathbf{A}) \Leftrightarrow \lambda \in \Sigma_{\text {ess }}\left(-\mathbf{L}_{0}+\mathbf{J}(\lambda)\right)$

Proof. Straightforward adaptation of [5, lemma 3.20] where it is shown that $\lambda \in \Sigma_{\text {ess, Engel }}(\mathbf{A}) \Leftrightarrow$ $\lambda \in \Sigma_{\text {ess, Engel }}\left(-\mathbf{L}_{0}+\mathbf{J}(\lambda)\right)$

Let us summarize the properties of the spectrum $\Sigma(\mathbf{A})$ of $\mathbf{A}$ :

Lemma 3.2 A satisfies the following properties:

1. its essential spectrum is: $\Sigma_{\text {ess }}(\mathbf{A})=\Sigma\left(-\mathbf{L}_{0}\right)$

2. $\Sigma(\mathbf{A})$ is at most countable.

3. $\Sigma(\mathbf{A})=\Sigma\left(-\mathbf{L}_{0}\right) \cup C V$. These sets are possibly non-disjoint.

4. For $\lambda \in \Sigma(\mathbf{A}) \backslash \Sigma\left(-\mathbf{L}_{0}\right), \lambda$ is an isolated eigenvalue and the generalized eigenspace $E_{\lambda}(\mathbf{A}) \equiv$ $\cup_{k} \operatorname{ker}(\lambda \mathrm{Id}-\mathbf{A})^{k}$ is finite dimensional. Hence $\Sigma(\mathbf{A}) \backslash \Sigma\left(-\mathbf{L}_{0}\right) \subset \Sigma_{p}(\mathbf{A})$.

5. Card $\Sigma(\mathbf{A}) \cap\{\lambda \in \mathbb{C}, \Re \lambda>-l\}<\infty$ where $l=\min _{i} l_{i}$.

Proof. 
1. From lemma $3.1 \lambda \in \Sigma_{\text {ess }}(\mathbf{A}) \Leftrightarrow \lambda \in \Sigma_{\text {ess }}\left(-\mathbf{L}_{0}+\mathbf{J}(\lambda)\right)$. Then, we apply [31, Theorem IV.5.26]. It shows that the essential spectrum does not change under compact perturbation. As $\mathbf{J}(\lambda) \in \mathcal{L}\left(\mathrm{L}^{2}\right)$ is compact, we find $\Sigma_{e s s}\left(-\mathbf{L}_{0}+\mathbf{J}(\lambda)\right)=\Sigma_{e s s}\left(-\mathbf{L}_{0}\right)$. Let us show that $\Sigma_{\text {ess }}\left(-\mathbf{L}_{0}\right)=\Sigma\left(-\mathbf{L}_{0}\right)$. The assertion " $\subset$ " is trivial. Now if $\lambda \in \Sigma\left(-\mathbf{L}_{0}\right)$, for example $\lambda=-l_{1}$, then $\lambda \mathrm{Id}+\mathbf{L}_{0}=\operatorname{diag}\left(0,-l_{1}+l_{2}, \ldots\right)$. Then $\mathcal{R}\left(\lambda \mathrm{Id}+\mathbf{L}_{0}\right)$ is closed and $\mathrm{L}^{2} \times\{0\} \times \ldots \times$ $\{0\} \subset \mathcal{N}\left(\lambda \mathrm{Id}+\mathbf{L}_{0}\right)$. Hence $\operatorname{dim} \mathcal{N}\left(\lambda \mathrm{Id}+\mathbf{L}_{0}\right)=\infty$. Also $\mathcal{R}\left(\lambda \mathrm{Id}+\mathbf{L}_{0}\right)=\{0\} \times \mathrm{L}^{2}\left(\Omega, \mathbb{R}^{\mathrm{p}-1}\right)$, hence $\operatorname{codim} \mathcal{R}\left(\lambda \mathrm{Id}+\mathbf{L}_{0}\right)=\infty$. Hence, according to definition A.9, $\lambda \in \Sigma_{\text {ess }}\left(-\mathbf{L}_{0}\right)$.

2-4. The assertions 2-4 are direct consequences of [31, Theorem IV.5.33].

5. If $\lambda=\rho+i \omega \in \Sigma(\mathbf{A})$ and $\rho>-l$, then $\lambda$ is a CV i.e. $\mathcal{N}\left(\operatorname{Id}-\left(\lambda \operatorname{Id}+\mathbf{L}_{0}\right)^{-1} \mathbf{J}(\lambda)\right) \neq \emptyset$ stating that $1 \in \Sigma_{p}\left(\left(\lambda \operatorname{Id}+\mathbf{L}_{0}\right)^{-1} \mathbf{J}(\lambda)\right)$. But $\left\|\left(\lambda \mathrm{Id}+\mathbf{L}_{0}\right)^{-1} \mathbf{J}(\lambda)\right\|\left\|_{\mathrm{L}^{2}} \leq\right\|\left\|\left(\lambda \mathrm{Id}+\mathbf{L}_{0}\right)^{-1} \mid\right\|_{\mathrm{L}^{2}}$. $\left\||| \mathbf{J}(\lambda)\left|\left\|_{L^{2}} \leq \frac{1}{\sqrt{\omega^{2}+(\rho+l)^{2}}}|| \mathbf{J}(\lambda)\right\|_{L^{2}} \leq \frac{1}{2}\right.\right.$ for $\lambda$ big enough since $\|\mid \mathbf{J}(\lambda)\|_{L^{2}}$ is bounded.

Hence, for $\lambda$ large enough $1 \notin \Sigma_{p}\left(\left(\lambda \mathrm{Id}+\mathbf{L}_{0}\right)^{-1} \mathbf{J}(\lambda)\right)$, which holds by the spectral radius inequality. This relationship states that the $\operatorname{CVs} \lambda$ satisfying $\Re \lambda>-l$ are located in a bounded set of the right part of $\mathbb{C}$; given that the elements of $\Sigma(\mathbf{A}) \backslash \Sigma\left(-\mathbf{L}_{0}\right)$ are isolated, there are a finite number of them.

We have shown that the CVs are almost all, i.e. except for possibly a finite number of them, located in the left part of the complex plane. This indicates that the unstable manifold is always finite dimensional in $\mathcal{X}^{(2)}$. The last result of this section is devoted to the regularity of the semigroup $\mathbf{T}(t)$ and an estimate of its operator norm. This is useful for the study of the inhomogeneous problem in section 4 .

Lemma 3.3 If $\mathbf{J} \in \mathrm{L}^{\infty}\left(\Omega^{2}, \mathbb{R}^{\mathrm{p}}\right)$, then the semigroup $(\mathbf{T}(t))_{t \geq 0}$ on $\mathcal{X}^{(2)}$ satisfies the following properties:

1. $(\mathbf{T}(t))_{t \geq 0}$ is norm continuous on $\mathcal{X}^{(2)}$ for $t>\tau_{m}$.

2. Let us define ${ }^{1} \epsilon \equiv \max \left(-l, \epsilon_{p}\right), \epsilon_{p} \equiv \max \Re(\Sigma(\mathbf{A}) \cap\{\lambda \in \mathbb{C}, \Re \lambda>-l\})$ and $l=\min _{i} l_{i}$. If $\epsilon<0$, then $\exists M \geq 1$ such that $\|\mid \mathbf{T}(t)\|_{\mathcal{X}^{(2)}} \leq M e^{\epsilon t / 2}, \forall t \geq 0$.

Proof.

1. This is consequence of lemma B.2 and [5, prop.4.3].

2. We use the result in [18, chapter 4, theorem 3.10 and corollary 3.12] for eventually norm continuous semigroups (see definition A.3 in appendix A) which links the growth bound of the semigroup to the spectrum of $\mathbf{A}$ :

$$
\inf \left\{w \in \mathbb{R}: \exists M_{w} \geq 1 \text { such that }\|\mid \mathbf{T}(t)\|_{\mathcal{X}^{(2)}} \leq M_{w} e^{w t}, \forall t \geq 0\right\}=\sup \Re \Sigma(\mathbf{A}) .
$$

From the previous lemma 3.2, we conclude the proof.

This lemma implies that the asymptotic stability of $\mathbf{U}=0$ in (13) is equivalent to the condition:

$$
\max \Re(\Sigma(\mathbf{A}) \cap\{\lambda \in \mathbb{C}, \Re \lambda>-l\})<0 .
$$

\footnotetext{
${ }^{1}$ where we set $\max \emptyset \equiv-\infty$.
} 


\subsection{Generalized eigenspaces}

Let us now compute the generalized eigenspaces of $\mathbf{A}$ (see [24, 1, 47, 15]). The proof of the next proposition closely follows [24] although it has to be adapted to our functional setting. A generalized eigenvector $\phi \neq 0$ is a vector which satisfies $(\lambda \operatorname{Id}-\mathbf{A})^{k} \phi=0$ for some integer $k$. The generalized eigenspace is then defined by $E_{\lambda}(\mathbf{A})=\cup_{i=1}^{\infty} \operatorname{ker}(\lambda \operatorname{Id}-\mathbf{A})^{i}$. As exposed in [24], a convenient way to characterize these spaces is the notion of Jordan chain (see definition A.10). Briefly, a Jordan chain $\left(\phi_{1}, \cdots, \phi_{m}\right) \in\left(\mathcal{X}^{(2)}\right)^{m+1}$ of length $m$ is an ordered set of vectors such that $\phi_{1} \neq 0$ and:

$$
\begin{aligned}
(\lambda \mathrm{Id}-\mathbf{A}) \phi_{1} & =0, \\
(\lambda \mathrm{Id}-\mathbf{A}) \phi_{i+1} & =\phi_{i}, 1 \leq i \leq m-1 .
\end{aligned}
$$

Hence, $\phi_{i}$ is in $\operatorname{ker}(\lambda \mathrm{Id}-\mathbf{A})^{i}$ and the first vector $\phi_{1}$ is always an eigenvector. Note that this implies that the $\phi_{i}$ are linearly independent. Conversely, given a generalized eigenvector $\phi$ such that $(\lambda \mathrm{Id}-\mathbf{A})^{m} \phi=0,(\lambda \mathrm{Id}-\mathbf{A})^{m-1} \phi \neq 0$, we can build a Jordan chain. Indeed, it is given (for example) by $\phi_{m}=\phi, \phi_{m-1}=(\lambda \mathrm{Id}-\mathbf{A}) \phi_{m} \cdots$. Finally, the generalized eigenspace is spanned by a finite number of Jordan chains.

The definition A.10 of the Jordan chain that we use is different, although equivalent, but a bit more convenient for the proofs of the next two propositions.

Proposition 3.4 For $\lambda_{0} \in \Sigma(\mathbf{A}) \backslash \Sigma\left(-\mathbf{L}_{0}\right)$ and $\forall m \geq 1$,

$$
\begin{aligned}
& \operatorname{ker}\left(\lambda_{0} \mathrm{Id}-\mathbf{A}\right)^{m}=\left\{\phi \in D(\mathbf{A}) \mid\left(\pi_{2} \phi\right)(\theta)=e^{\lambda_{0} \theta} \sum_{i=0}^{m-1} \frac{\theta^{i}}{i !} \mathbf{U}_{m-1-i}\right. \\
&\text { where } \left.\left(\mathbf{U}_{0}, \cdots, \mathbf{U}_{m-1}\right) \text { is a Jordan chain (see definition A.10) for } \Delta\left(\lambda_{0}\right)\right\}
\end{aligned}
$$

Proof. Let us define the following operator: $\mathbf{A}_{0} \phi=\frac{d}{d \theta} \phi$ with domain

$$
D\left(\mathbf{A}_{0}\right)=\left\{\phi \in W^{1,2}\left(-\tau_{m}, 0 ; \mathrm{L}^{2}\right) \mid \phi(0)=0\right\}
$$

and the exponential function $\epsilon_{\lambda}(\theta)=e^{\lambda \theta}$. From [5][Lemma 3.20]:

$$
\forall \lambda \quad\left[\begin{array}{cc}
\Delta(\lambda) & 0 \\
0 & \lambda \mathrm{Id}-\mathbf{A}_{0}
\end{array}\right]=\mathbf{F}(\lambda)(\lambda \mathrm{Id}-\mathbf{A}) \mathbf{E}(\lambda)
$$

where $\mathbf{F}(\lambda)=\left[\begin{array}{cc}\mathrm{Id} & \mathbf{L}_{1} R\left(\lambda, \mathbf{A}_{0}\right) \\ 0 & \mathrm{Id}\end{array}\right] \in \mathcal{L}\left(\mathcal{X}^{(2)}\right)$ and $\mathbf{E}(\lambda)=\left[\begin{array}{cc}\mathrm{Id} & 0 \\ \epsilon_{\lambda} \otimes \mathrm{Id} & \mathrm{Id}\end{array}\right] \in \mathcal{L}\left(\mathcal{X}^{(2)}\right)$ are invertible operators with $\left(\epsilon_{\lambda} \otimes \mathrm{Id}\right) x \equiv \epsilon_{\lambda} x \in \mathrm{L}^{2}\left(\left[-\tau_{\mathrm{m}}, 0\right], \mathrm{L}^{2}\right)$ and $R\left(\lambda, \mathbf{A}_{0}\right)$ is the resolvent of $\mathbf{A}_{0}$. The rest of the proof is the same as in [24][Theorem 4.2].

Remark 2 A characterization of the Jordan chains of $\Delta(\lambda)$ is given in lemma A.12.

Given $\lambda \in \Sigma_{p}(\mathbf{A})$ and the finite dimensional generalized eigenspace $E_{\lambda}(\mathbf{A})=\cup_{i=1}^{\infty} \operatorname{ker}(\lambda \mathrm{Id}-$ $\mathbf{A})^{i}$, we want to find the spectral projector $P_{\lambda}$ on $E_{\lambda}(\mathbf{A})$ which commutes with $\mathbf{A}$. As $\mathcal{X}^{(2)}$ is a Hilbert space and $\mathbf{A}$ is densely defined, we can define the adjoint $\mathbf{A}^{*}$ of $\mathbf{A}$, then $\bar{\lambda} \in \Sigma_{p}\left(\mathbf{A}^{*}\right)$ and 
$\operatorname{dim} E_{\lambda}(\mathbf{A})=\operatorname{dim} E_{\bar{\lambda}}\left(\mathbf{A}^{*}\right) \equiv m_{\lambda}$. Thus, if $\left(\phi_{1}, \cdots, \phi_{m_{\lambda}}\right)$ is a basis of $E_{\lambda}(\mathbf{A})$ and $\left(\phi_{1}^{*}, \cdots, \phi_{m_{\lambda}}^{*}\right)$ is a basis of $E_{\bar{\lambda}}\left(\mathbf{A}^{*}\right)$ such that $\left\langle\phi_{i}^{*}, \phi_{j}\right\rangle_{\mathcal{X}^{(2)}}=\delta_{i j}$, a spectral projector is given by:

$$
\forall u \in \mathcal{X}, \quad P_{\lambda} u \equiv \sum_{i=1}^{m_{\lambda}}\left\langle\phi_{i}^{*}, u\right\rangle_{\mathcal{X}^{(2)}} \phi_{i}
$$

Notice that $P_{\lambda}$ commutes with $\mathbf{A}$.

\subsection{Spectral projector on generalized eigenspaces}

In order to derive the center manifold theorem in section 4, we need to know how to project any vector of the state space $\mathcal{X}^{(2)}$ onto the generalized eigenspaces computed in section 3.1. The expression (22) of the spectral projector $P_{\lambda}$ for an eigenvalue $\lambda$ is impractical as it requires to compute the generalized eigenspace of the adjoint operator. We show in this section that the inner products $\left\langle\phi_{i}^{*}, u\right\rangle_{\mathcal{X}^{(2)}}$, required to obtain $P_{\lambda}$, can be computed without explicitly computing $\mathbf{A}^{*}$. By following closely the proof of [24, Theorem 5.1] adapted in order to fit to our functional setting by using the tools of [5], we are able to prove proposition 3.8. This proposition requires the proof of the following two lemmas and is the basis of the upcoming computation of the reduced equation.

Let us define the operator $\mathbf{A}_{0}$ on $\mathrm{L}^{2}\left(-\tau_{\mathrm{m}}, 0 ; \mathrm{L}^{2}\right)$ by $\mathbf{A}_{0} \phi=\frac{d}{d \theta} \phi$ with domain $D\left(\mathbf{A}_{0}\right)=$ $\left\{\phi \in W^{1,2}\left(-\tau_{m}, 0 ; \mathrm{L}^{2}\right) \mid \phi(0)=0\right\}$. The resolvent of $\mathbf{A}_{0}$ is easily found using a variation-ofconstants formula:

$$
\left[R\left(\lambda, \mathbf{A}_{0}\right) \phi\right](\theta)=\int_{\theta}^{0} e^{\lambda(\theta-s)} \phi(s) d s
$$

Notice that $\Sigma\left(\mathbf{A}_{0}\right)=\emptyset$. An integration-by-parts gives the adjoint of $R\left(\lambda, \mathbf{A}_{0}\right)$

$$
\left[R\left(\lambda, \mathbf{A}_{0}\right)^{*} \phi\right](\theta)=\int_{-\tau_{m}}^{\theta} e^{-\bar{\lambda}(\theta-s)} \phi(s) d s
$$

The next lemma is used in the proof of proposition 3.8 .

Lemma 3.5 If $\mathbf{F}(\lambda)=\left[\begin{array}{cc}\operatorname{Id} & \mathbf{L}_{1} R\left(\lambda, \mathbf{A}_{0}\right) \\ 0 & \text { Id }\end{array}\right] \in \mathcal{L}\left(\mathcal{X}^{(2)}\right)$, then we have

$$
\mathbf{F}(\lambda)^{*}=\left[\begin{array}{cc}
\mathrm{Id} & 0 \\
\mathbf{J}[\cdot]^{*}-\bar{\lambda} R\left(\lambda, \mathbf{A}_{0}\right)^{*} \mathbf{J}[\cdot]^{*} & \mathrm{Id}
\end{array}\right]
$$

where $\mathbf{J}[s]^{*}$ is the adjoint in $\mathrm{L}^{2}$ of $\mathbf{J}[s], \mathbf{J}[s]$ being the integral operator on $\mathrm{L}^{2}$ with kernel $\forall s \in$ $\left[-\tau_{m}, 0\right], J_{i j}\left(\mathbf{r}, \mathbf{r}^{\prime}\right)[s] \equiv J_{i j}\left(\mathbf{r}, \mathbf{r}^{\prime}\right) H\left(s+\tau_{i j}\left(\mathbf{r}, \mathbf{r}^{\prime}\right)\right.$ ) (see also lemma B.1 for a definition of $\left.\mathbf{J}[s]\right)$. 
Proof. Let us compute the following scalar product:

$$
\begin{gathered}
\left\langle\left[\begin{array}{c}
y \\
\psi
\end{array}\right], \mathbf{F}(\lambda)\left[\begin{array}{l}
x \\
\phi
\end{array}\right]\right\rangle_{\mathcal{X}^{(2)}}=\langle y, x\rangle_{\mathrm{L}^{2}}+\int_{-\tau_{m}}^{0}\langle\psi(s), \phi(s)\rangle_{\mathrm{L}^{2}} d s+\left\langle y, \mathbf{L}_{1} R\left(\lambda, \mathbf{A}_{0}\right) \phi\right\rangle_{\mathrm{L}^{2}} \\
=\langle y, x\rangle_{\mathrm{L}^{2}}+\int_{-\tau_{m}}^{0}\langle\psi(s), \phi(s)\rangle_{\mathrm{L}^{2}} d s+\left\langle y, \mathbf{J}\left[R\left(\lambda, \mathbf{A}_{0}\right) \phi\right](0)\right\rangle_{\mathrm{L}^{2}}-\int_{-\tau_{m}}^{0}\left\langle y, \mathbf{J}[s] \frac{d}{d s}\left[R\left(\lambda, \mathbf{A}_{0}\right) \phi\right](s) d s\right\rangle_{\mathrm{L}^{2}} \\
=\langle y, x\rangle_{\mathrm{L}^{2}}+\int_{-\tau_{m}}^{0}\langle\psi(s), \phi(s)\rangle_{\mathrm{L}^{2}} d s-\int_{-\tau_{m}}^{0}\left\langle\mathbf{J}[s]^{*} y,\left[\mathbf{A}_{0} R\left(\lambda, \mathbf{A}_{0}\right) \phi\right](s) d s\right\rangle_{\mathrm{L}^{2}} \\
=\langle y, x\rangle_{\mathrm{L}^{2}}+\int_{-\tau_{m}}^{0}\langle\psi(s), \phi(s)\rangle_{\mathrm{L}^{2}} d s-\int_{-\tau_{m}}^{0}\left\langle\mathbf{J}[s]^{*} y,\left[-\phi+\lambda R\left(\lambda, \mathbf{A}_{0}\right) \phi\right](s) d s\right\rangle_{\mathrm{L}^{2}} \\
=\langle y, x\rangle_{\mathrm{L}^{2}}+\int_{-\tau_{m}}^{0}\left\langle\psi(s)+\mathbf{J}[s]^{*} y, \phi(s)\right\rangle_{\mathrm{L}^{2}} d s-\int_{-\tau_{m}}^{0}\left\langle\bar{\lambda} R\left(\lambda, \mathbf{A}_{0}\right)^{*} \mathbf{J}[s]^{*} y, \phi(s)\right\rangle_{\mathrm{L}^{2}} d s
\end{gathered}
$$

The identification of the adjoint is now straightforward.

Using the previous lemma, the proof of the next proposition is very close to the one of proposition 3.4. It consists in showing that the generalized eigenvectors of $\mathbf{A}^{*}$ are closely related to the Jordan chains of $\lambda \rightarrow \Delta(\lambda)^{*}$. Let us first define $\mathbf{F}^{T}$ on $D(\mathbf{A})$ by:

$$
\mathbf{F}^{T}\left[\begin{array}{l}
x \\
\phi
\end{array}\right] \equiv\left[\begin{array}{c}
x \\
\mathbf{J}^{*}[\theta] \phi(0)-\int_{-\tau_{m}}^{\theta}
\end{array}\right.
$$

Before stating the main result of this section, we also define the following bilinear product:

Definition 3.6 The bilinear product $\langle\langle\psi, u\rangle\rangle$ for $\psi, u \in \mathcal{X}^{(2)}$ reads:

$$
\langle\langle\psi, u\rangle\rangle \equiv\left\langle\pi_{1} \psi, \pi_{1} u\right\rangle_{\mathrm{L}^{2}}+\int_{\Omega^{2}} d \mathbf{r} d \overline{\mathbf{r}} \sum_{i j} \int_{-\tau_{i j}(\mathbf{r}, \overline{\mathbf{r}})}^{0}\left(\pi_{2} \psi\right)_{i}\left(\mathbf{r},-s-\tau_{i j}(\mathbf{r}, \overline{\mathbf{r}})\right) \tilde{J}_{i j}(\mathbf{r}, \overline{\mathbf{r}})\left(\pi_{2} u\right)_{j}(\overline{\mathbf{r}}, s) d s
$$

where

$$
\tilde{\mathbf{J}} \equiv \mathbf{J}(0)
$$

This is written:

$$
\left\langle\pi_{1} \psi, \pi_{1} u\right\rangle_{\mathrm{L}^{2}}+\int_{\Omega^{2}} d \mathbf{r} d \overline{\mathbf{r}} \int_{-\boldsymbol{\tau}(\mathbf{r}, \overline{\mathbf{r}})}^{0}\left\langle\left(\pi_{2} \psi\right)(\mathbf{r},-s-\boldsymbol{\tau}(\mathbf{r}, \overline{\mathbf{r}})), \tilde{\mathbf{J}}(\mathbf{r}, \overline{\mathbf{r}})\left(\pi_{2} u\right)(\overline{\mathbf{r}}, s)\right\rangle_{\mathbb{R}^{p}} d s .
$$

It is straightforward to check that:

Lemma 3.7 The bilinear product $\langle\langle\rangle$,$\rangle is symmetric if \tilde{J}_{i j}(\mathbf{r}, \overline{\mathbf{r}})=\tilde{J}_{j i}(\overline{\mathbf{r}}, \mathbf{r})$ and $\tau_{i j}(\mathbf{r}, \overline{\mathbf{r}})=$ $\tau_{j i}(\overline{\mathbf{r}}, \mathbf{r})$.

Using the bilinear product, we find an expression of the spectral projector $P_{\lambda}$.

Proposition 3.8 1. For $\bar{\lambda}_{0} \in \Sigma\left(\mathbf{A}^{*}\right) \backslash \Sigma\left(-\mathbf{L}_{0}^{*}\right)$ and $\forall m \geq 1$, we have as in proposition 3.4: $\operatorname{ker}\left(\bar{\lambda}_{0} \mathrm{Id}-\mathbf{A}^{*}\right)^{m}=\left\{\mathbf{F}^{T} \psi, \psi \in D(\mathbf{A}) \mid\left(\pi_{2} \psi\right)(\theta)=e^{\bar{\lambda}_{0} \theta} \sum_{i=0}^{m-1} \frac{\theta^{i}}{i !} \mathbf{U}_{m-1-i}^{*}\right.$ where $\left(\mathbf{U}_{0}^{*}, \cdots, \mathbf{U}_{m-1}^{*}\right)$ is a Jordan chain (see definition A.10) for $\left.\Delta\left(\bar{\lambda}_{0}\right)^{*}\right\}$ 
2. For $\lambda_{0} \in \Sigma(\mathbf{A}) \backslash \Sigma\left(-\mathbf{L}_{0}\right)$, choose a basis $\left(\phi_{i}\right)_{i=1 \cdots m_{\lambda_{0}}}\left(\right.$ resp. $\left.\left(\phi_{i}^{*}\right)_{i=1 \cdots m_{\lambda_{0}}}\right)$ of $E_{\lambda_{0}}(\mathbf{A})$ as in proposition 3.4 (resp. $E_{\bar{\lambda}_{0}}\left(\mathbf{A}^{*}\right)$ ) such that $\left\langle\phi_{i}^{*}, \phi_{j}\right\rangle_{\mathcal{X}^{(2)}}=\delta_{i j}$. Then, $\forall i \leq m_{\lambda_{0}}$, there are $\psi_{i} \in D(\mathbf{A})$ as above such that $\forall u \in \mathcal{X}^{(2)}$ :

$$
\left\langle\phi_{i}^{*}, u\right\rangle_{\mathcal{X}^{(2)}}=\left\langle\left\langle\psi_{i}, u\right\rangle\right\rangle
$$

where $\left(\pi_{2} \psi_{i}\right)(\theta)=e^{\bar{\lambda}_{0} \theta} \sum_{r=0}^{m_{i}-1} \frac{\theta^{r}}{r !} \mathbf{U}_{i ; m_{i}-1-r}^{*}$. Then, the projector $P_{\lambda_{0}}$ is given by:

$$
\forall u \in \mathcal{X}^{(2)}, \quad P_{\lambda_{0}} u=\sum_{i=1}^{m_{\lambda_{0}}}\left\langle\left\langle\psi_{i}, u\right\rangle\right\rangle \phi_{i}
$$

Proof. From [5][Lemma 3.20]: $\left[\begin{array}{cc}\Delta(\lambda) & 0 \\ 0 & \lambda \mathrm{Id}-\mathbf{A}_{0}\end{array}\right]=\mathbf{F}(\lambda)(\lambda \mathrm{Id}-\mathbf{A}) \mathbf{E}(\lambda)$ with $\mathbf{F}(\lambda)=$ $\left[\begin{array}{cc}\operatorname{Id} & \mathbf{L}_{1} R\left(\lambda, \mathbf{A}_{0}\right) \\ 0 & \operatorname{Id}\end{array}\right] \in \mathcal{L}\left(\mathcal{X}^{(2)}\right)$ and $\mathbf{E}(\lambda)=\left[\begin{array}{cc}\operatorname{Id} & 0 \\ \epsilon_{\lambda} \otimes \operatorname{Id} & \operatorname{Id}\end{array}\right] \in \mathcal{L}\left(\mathcal{X}^{(2)}\right)$, we find:

$$
\left[\begin{array}{cc}
\Delta(\lambda)^{*} & 0 \\
0 & \left(\lambda \mathrm{Id}-\mathbf{A}_{0}\right)^{*}
\end{array}\right]=\mathbf{E}(\lambda)^{*}\left(\bar{\lambda} \mathrm{Id}-\mathbf{A}^{*}\right) \mathbf{F}(\lambda)^{*}
$$

Proof of 1 .

Similarly to the proof of proposition 3.4, the Jordan chains of $\bar{\lambda} \mathrm{Id}-\mathbf{A}^{*}$ at $\bar{\lambda}=\bar{\lambda}_{0}$ are in oneto-one correspondence with the Jordan chains of $\Delta(\lambda)^{*}$ at $\bar{\lambda}=\bar{\lambda}_{0}$. The proof reduces to finding the Jordan chains $\phi^{*}$ of $\mathbf{A}^{*}$. Take a Jordan chain $\left(\mathbf{U}_{0}, \cdots, \mathbf{U}_{m-1}\right)$ of $\Delta(\lambda)^{*}$ at $\bar{\lambda}=\bar{\lambda}_{0}$, then from $(23), \mathbf{F}(\lambda)^{*}\left[\begin{array}{c}\alpha^{*}(\bar{\lambda}) \\ 0\end{array}\right]$ is a root function for $\mathbf{A}^{*}$ where $\alpha^{*}(\bar{\lambda})=\sum_{i=0}^{m-1}\left(\bar{\lambda}-\bar{\lambda}_{0}\right)^{i} \mathbf{U}_{i}$. Computing $\pi_{2} \mathbf{F}(\lambda)^{*}\left[\begin{array}{c}\alpha^{*}(\bar{\lambda}) \\ 0\end{array}\right]$ using lemma 3.5, we find up to orders $O\left(\left(\bar{\lambda}-\bar{\lambda}_{0}\right)^{m}\right)$ :

$$
\begin{aligned}
& \mathbf{J}^{*}(\theta) \alpha^{*}(\bar{\lambda})-\int_{-\tau_{m}}^{\theta} \bar{\lambda} e^{-\bar{\lambda}(\theta-s)} \mathbf{J}[s]^{*} \alpha^{*}(\bar{\lambda}) d s= \\
& \mathbf{J}^{*}(\theta) \alpha^{*}(\bar{\lambda})-\int_{-\tau_{m}}^{\theta} \mathbf{J}[s]^{*} \frac{d}{d s}\left[e^{-\bar{\lambda}(\theta-s)} \alpha(\bar{\lambda})\right] d s= \\
& \\
& \sum_{l=0}^{m-1}\left(\bar{\lambda}-\bar{\lambda}_{0}\right)^{l}\left\{\mathbf{J}^{*}(\theta) \mathbf{U}_{l}-\int_{-\tau_{m}}^{\theta} \mathbf{J}[s]^{*} \frac{d}{d s}\left[e^{\bar{\lambda}_{0}(s-\theta)} \sum_{j=0}^{l} \mathbf{U}_{l-j} \frac{(s-\theta)^{j}}{j !}\right]\right\} d s
\end{aligned}
$$

Hence, we find that

$$
\mathbf{F}(\lambda)^{*}\left[\begin{array}{c}
\alpha^{*}(\bar{\lambda}) \\
0
\end{array}\right]=\sum_{l=0}^{m-1}\left(\bar{\lambda}-\bar{\lambda}_{0}\right)^{l} \mathbf{F}^{T} \psi_{l}
$$

where $\psi_{l}=\left[\begin{array}{c}\mathbf{U}_{l}^{*} \\ e^{\bar{\lambda}_{0} \theta} \sum_{j=0}^{l} \mathbf{U}_{l-j}^{*} \frac{\theta^{j}}{j !}\end{array}\right] \in D(\mathbf{A})$. We have found that the generalized eigenvectors of $\mathbf{A}^{*}$ are given by the $\mathbf{F}^{T} \psi_{l}$.

Proof of 2 .

$R R n^{\circ} 8020$ 
Let $\phi^{*}$ be a generalized eigenvector of $\mathbf{A}$. According to 1., we can write $\phi^{*}=\mathbf{F}^{T}\left[\begin{array}{l}x \\ \phi\end{array}\right]$. For the computation of the projector $P_{\lambda}$, we need to compute the scalar product $\left\langle u, \phi^{*}\right\rangle_{\mathcal{X}^{(2)}}$.

$$
\begin{aligned}
& \left\langle u, \phi^{*}\right\rangle_{\mathcal{X}^{(2)}}=\left\langle\left[\begin{array}{l}
y \\
\psi
\end{array}\right], \mathbf{F}^{T}\left[\begin{array}{l}
x \\
\phi
\end{array}\right]\right\rangle_{\mathcal{X}^{(2)}} \equiv\langle y, x\rangle_{\mathrm{L}^{2}}+\int_{-\tau_{m}}^{0} d \theta\left\langle\psi(\theta), \mathbf{J}[\theta]^{*} \phi(0)\right\rangle_{\mathrm{L}^{2}} \\
& -\int_{-\tau_{m}}^{0} d \theta \int_{-\tau_{m}}^{\theta} d s\left\langle\psi(\theta), \mathbf{J}[s]^{*} \frac{d}{d s}[\phi(s-\theta)]\right\rangle \\
& =\langle y, x\rangle_{\mathrm{L}^{2}}+\int_{-\tau_{m}}^{0} d \theta\left\langle\psi(\theta), \mathbf{J}[\theta]^{*} \phi(0)\right\rangle_{\mathrm{L}^{2}} \\
& -\int_{-\tau_{m}}^{0} d \theta \int_{\Omega^{2}} d \mathbf{r} d \overline{\mathbf{r}} \int_{-\tau_{m}}^{\theta} d s\left\langle\psi(\mathbf{r}, \theta), \mathbf{J}^{*}(\mathbf{r}, \overline{\mathbf{r}})^{*} \dot{\phi}(\overline{\mathbf{r}}, s-\theta)\right\rangle_{\mathbb{R}^{p}} H(s+\boldsymbol{\tau}(\mathbf{r}, \overline{\mathbf{r}})) \\
& =\langle y, x\rangle_{\mathrm{L}^{2}}+\int_{-\tau_{m}}^{0} d \theta\left\langle\psi(\theta), \mathbf{J}[\theta]^{*} \phi(0)\right\rangle_{\mathrm{L}^{2}} \\
& -\sum_{i j} \int_{\Omega^{2}} d \mathbf{r} \tilde{J}_{i j}(\mathbf{r}, \overline{\mathbf{r}}) d \overline{\mathbf{r}} \int_{-\tau_{m}}^{0} d \theta \int_{-\tau_{m}}^{\theta} d s \psi_{j}(\mathbf{r}, \theta) \dot{\phi}_{i}(\overline{\mathbf{r}}, s-\theta) H\left(s+\tau_{i j}(\mathbf{r}, \overline{\mathbf{r}})\right) \\
& =\langle y, x\rangle_{\mathrm{L}^{2}}+\int_{-\tau_{m}}^{0} d \theta\left\langle\psi(\theta), \mathbf{J}[\theta]^{*} \phi(0)\right\rangle_{\mathrm{L}^{2}} \\
& -\sum_{i j} \int_{\Omega^{2}} d \mathbf{r} \tilde{J}_{i j}(\mathbf{r}, \overline{\mathbf{r}}) d \overline{\mathbf{r}} \int_{-\tau_{i j}(\mathbf{r}, \overline{\mathbf{r}})}^{0} d \theta \int_{-\tau_{i j}(\mathbf{r}, \overline{\mathbf{r}})}^{\theta} d s \psi_{j}(\mathbf{r}, \theta) \dot{\phi}_{i}(\overline{\mathbf{r}}, s-\theta) \\
& =\langle y, x\rangle_{\mathrm{L}^{2}}+\sum_{i j} \int_{\Omega^{2}} d \mathbf{r} \tilde{J}_{i j}(\mathbf{r}, \overline{\mathbf{r}}) d \overline{\mathbf{r}} \int_{-\tau_{i j}(\mathbf{r}, \overline{\mathbf{r}})}^{0} d s \psi_{j}(\mathbf{r}, \theta) \phi_{i}\left(\overline{\mathbf{r}}, s-\tau_{i j}(\mathbf{r}, \overline{\mathbf{r}})\right)
\end{aligned}
$$

where $H$ is the Heaviside function. The last line is equal to the definition of $\left\langle\left\langle\left[\begin{array}{l}x \\ \phi\end{array}\right], u\right\rangle\right\rangle$. The proof is complete.

We have found an analytical expression for the spectral projector $P_{\lambda}$. We can use this result to obtain a decomposition (see [49] p. 228) of the history space for the eigenvalue $\lambda \in \Sigma_{p}(\mathbf{A})$ of algebraic multiplicity $m_{\lambda}$ :

$$
\mathcal{X}^{(2)}=\operatorname{ker}(\lambda \operatorname{Id}-\mathbf{A})^{m_{\lambda}} \oplus \operatorname{Im}(\lambda \operatorname{Id}-\mathbf{A})^{m_{\lambda}}=E_{\lambda}(\mathbf{A}) \oplus \operatorname{Im}(\lambda \operatorname{Id}-\mathbf{A})^{m_{\lambda}},
$$

the last equality comes from $\operatorname{ker}(\lambda \mathrm{Id}-\mathbf{A})^{m_{\lambda}}=E_{\lambda}(\mathbf{A})$. From the general formula $\operatorname{ker}(\bar{\lambda} \operatorname{Id}-$ $\left.\mathbf{A}^{*}\right)^{m_{\lambda}}=\left(\operatorname{Im}(\lambda \operatorname{Id}-\mathbf{A})^{m_{\lambda}}\right)^{\perp}$, we obtain that

$$
\operatorname{Im}(\lambda \operatorname{Id}-\mathbf{A})^{m_{\lambda}}=\left\{\phi \in \mathcal{X}^{(2)} \mid \forall \psi \in E_{\bar{\lambda}}\left(\mathbf{A}^{*}\right),\langle\psi, \phi\rangle_{\mathcal{X}^{(2)}}=0\right\} .
$$

\subsection{Phase space decomposition}

We end this section on linear analysis with a decomposition of the history space according to the eigenvalues of the infinitesimal generator $\mathbf{A}$. This will be useful for the derivation of the center manifold theorem in section 4. Let us write a splitting of the spectrum of $\mathbf{A}$ according to the real part of the eigenvalues:

$$
\Sigma_{u} \equiv \Sigma(\mathbf{A}) \cap \mathbb{C}_{+}, \Sigma_{c} \equiv \Sigma_{p}(\mathbf{A}) \cap i \mathbb{R}, \Sigma_{s} \equiv \Sigma(\mathbf{A}) \cap \mathbb{C}_{-}
$$


where we have written $\mathbb{C}_{-}=\{z \in \mathbb{C} \mid \Re z<0\}$ and $\mathbb{C}_{+}=\{z \in \mathbb{C} \mid \Re z>0\}$. It was shown in lemma 3.2 that: $\forall a>\min _{i} l_{i}, \operatorname{Card} \Sigma(\mathbf{A}) \cap(a,+\infty)_{\mathbb{C}}<\infty$. Hence, we can find a spectral gap $\gamma>0$ such that

$$
\max \Re \Sigma_{s}<-\gamma, \min \Re \Sigma_{u}>\gamma
$$

As $\Sigma_{c}$ and $\Sigma_{s}$ are finite and included in the point spectrum $\Sigma_{p}(\mathbf{A})$ of $\mathbf{A}$, we can use (25) to obtain a decomposition of $\mathcal{X}^{(2)}$ according to the splitting of the spectrum:

$$
\mathcal{X}^{(2)}=\mathcal{X}_{u}^{(2)} \oplus \mathcal{X}_{c}^{(2)} \oplus \mathcal{X}_{s}^{(2)}
$$

where the spaces $\mathcal{X}_{u}^{(2)}, \mathcal{X}_{c}^{(2)}, \mathcal{X}_{s}^{(2)}$ are invariant by $\mathbf{A}$ and $\mathbf{T}(t)$ for all $t \geq 0$. For example, the center part, $\mathcal{X}_{c}=\bigoplus_{\lambda \in \Sigma_{c}} E_{\lambda}(\mathbf{A})$. We write $P_{c}=\sum_{\lambda \in \Sigma_{c}} P_{\lambda}, P_{u}=\sum_{\lambda \in \Sigma_{u}} P_{\lambda}$ the spectral projectors on $\mathcal{X}_{c}^{(2)}, \mathcal{X}_{u}^{(2)}$ and $P_{s}=\mathrm{Id}-P_{c}-P_{u}$. We also write $\mathbf{A}_{u}, \mathbf{A}_{c}, \mathbf{A}_{s}$ (resp. $\mathbf{T}_{u}, \mathbf{T}_{c}, \mathbf{T}_{s}$ ) the restriction of $\mathbf{A}$ (resp. $\mathbf{T})$ to the different subspaces. Notice that $\Sigma\left(\mathbf{A}_{i}\right)=\Sigma_{i}, i=u, c, s$. The spectral mapping theorem (see [18] and theorem A.4) gives:

$$
\left\|\left|\mathbf{T}_{s}(t)\right|\right\|_{\mathcal{X}^{(2)}} \leq M e^{-\gamma t}, t \geq 0
$$

where $M \geq 1$ is a constant. As $\mathcal{X}_{u}^{(2)}$ is finite dimensional, there is a matrix such that $\mathbf{T}_{u}(t)=e^{t A_{u}}$ (see for example [18]). Thus, $\mathbf{T}_{u}$ can be extended to a group such that:

$$
\left\|\mathbf{T}_{u}(t)\right\|_{\mathcal{X}^{(2)}} \leq M e^{-\gamma|t|}, t \leq 0
$$

Finally, we define the hyperbolic projector $P_{h}=P_{u}+P_{s}$ and associated operators $\mathbf{A}_{h}=\mathbf{A} P_{h}$ and $\mathbf{T}_{h}=\mathbf{T} P_{h}$.

\section{Center manifold reduction}

In the case where $\Sigma_{c} \neq \emptyset$, our program is to study the nonlinear dynamics of (4), using the tools of [25]. We find a manifold, called the center manifold, which is invariant by the dynamics of (4) and which is (exponentially) attractive if $\Sigma_{u}(\mathbf{A})=\emptyset$. From lemma 3.2, the center part $\mathcal{X}_{c}^{(2)}$ of the history space is finite dimensional. As it has the same dimension as the center manifold, it follows that the center manifold is also finite dimensional. Hence, when studying the neural fields equations with a non empty center part, we can restrict the study to the center manifold, which allows to describe without approximation the local non trivial dynamics with a finite dimensional system. In this article, a bifurcation is said to occur when the center part changes.

The study of the center manifold theorem in infinite dimension has led to some 'optimal' requirements to make the theorem work.

1. The first is to write the dynamical system as an abstract Cauchy problem. More precisely, we need to find three Banach spaces $\mathcal{Z} \hookrightarrow \mathcal{Y} \hookrightarrow \mathcal{X}$ with continuous embeddings such that our equations can be rewritten as

$$
\frac{d}{d t} u=\mathbf{A} u+\mathbf{R}(u)
$$

where $\mathbf{A}$ is a continuous linear operator in $\mathcal{L}(\mathcal{Z}, \mathcal{X})$ and $\mathbf{R} \in C^{k}(\mathcal{V}, \mathcal{Y}), k \geq 2$, is a nonlinear function defined on a neighbourhood $\mathcal{V} \subset \mathcal{Z}$ of 0 satisfying $\mathbf{R}(0)=0$ and $D \mathbf{R}(0)=0$.

2. Then the spectrum of the linear operator $\mathbf{A}$ needs to have a spectral decomposition with finite dimensional central part $\mathcal{X}_{c}$ and a spectral gap $\gamma$. This gives a finite dimensional center manifold and well defined convergence/divergence rates to/from the center manifold. 
3. Finally, the linear system

$$
\frac{d}{d t} u=\mathbf{A} u+F(t)
$$

has to feature exponentially bounded solutions for $t \in \mathbb{R}$ for some exponentially bounded function $F$ defined on $\mathbb{R}$. This will be explained more precisely below. This last property is needed to give some regularity to the center manifold (it has the same regularity as $\mathbf{R}$ ) and also to prove its existence.

We check the conditions in the next subsections.

\subsection{Formulation as a Cauchy problem}

We start by writing (6) as in (26). We choose a stationary solution $\mathbf{V}^{f}$ and $\mathbf{A}$ as in (16). Let us consider a parameter $\mu$, for example it can be the nonlinear gain $\sigma$, the threshold $h$ or the pair $(\sigma, h)$ : we assume that $\mu \in \mathbb{R}^{m_{\text {par }}}, m_{\text {par }}$ being the number of parameters. Notice that the equilibrium $\mathbf{V}^{f}$ may depend on $\mu$. It is such that when $\mu=\mu_{c}$, then the punctual spectrum contains purely imaginary eigenvalues. We write the equation (9) for a perturbation $\mathbf{U}$ of $\mathbf{V}^{f}$ where $\mathbf{V}(t)=\mathbf{V}^{f}+\mathbf{U}(t)$ :

$$
\dot{\mathbf{U}}(t)=-\mathbf{L}_{0} \mathbf{U}(t)+\mathbf{L}_{1}(\mu) \cdot\left(\mathbf{S}\left(\mathbf{U}_{t}+\mathbf{V}^{f}\right)-\mathbf{S}\left(\mathbf{V}^{f}\right)\right)
$$

Using a Taylor expansion with integral remainder (we have also made the dependency of $\mathbf{V}^{f}$ on the parameter $\mu$ implicit), we find:

$$
\begin{aligned}
& -\mathbf{L}_{0} \mathbf{U}(t)+\mathbf{L}_{1}(\mu) \cdot\left(\mathbf{S}\left(\mathbf{U}_{t}+\mathbf{V}^{f}\right)-\mathbf{S}\left(\mathbf{V}^{f}\right)\right)= \\
& \left(-\mathbf{L}_{0}+\tilde{\mathbf{L}}_{1}(\mu)\right) \cdot \mathbf{U}_{t}+\mathbf{L}_{1}(\mu) \cdot \int_{0}^{1}(1-s) \mathbf{S}^{(2)}\left(\mathbf{V}^{f}+s \mathbf{U}_{t}\right) \mathbf{U}_{t}^{2} d s \\
& \equiv \mathbf{L}(\mu) \mathbf{U}_{t}+\mathbf{L}_{1}(\mu) \mathbf{G}(\mathbf{U})
\end{aligned}
$$

In order to use [25, Theorem 2.3.3], i.e. the parameter dependent center manifold, we write the previous equation:

$$
\mathbf{L}(\mu) \mathbf{U}_{t}+\mathbf{L}_{1}(\mu) \mathbf{G}(\mathbf{U})=\mathbf{L}\left(\mu_{c}\right) \mathbf{U}_{t}+\left(\mathbf{L}_{1}(\mu) \mathbf{G}(\mathbf{U})+\left(\mathbf{L}(\mu)-\mathbf{L}\left(\mu_{c}\right)\right) \mathbf{U}_{t}\right) .
$$

We take a classical solution $\mathbf{U}(t)$ of $(28)$, i.e. a function $\mathbf{U} \in C^{0}\left(\left[-\tau_{m}, \infty\right), \mathrm{L}^{2}\right) \cap \mathrm{C}^{1}\left([0, \infty), \mathrm{L}^{2}\right)$ such that $\mathbf{U}_{t} \in W^{1,2}\left(-\tau_{m}, 0 ; \mathrm{L}^{2}\right)$ and $\mathbf{U}$ satisfies (28). The history segment $u(t) \equiv\left[\begin{array}{c}\mathbf{U}(t) \\ \mathbf{U}_{t}\end{array}\right]$ satisfies:

$$
\left\{\begin{array}{l}
\dot{u}(t)=\mathbf{A} u(t)+\mathbf{R}(u(t), \mu) \\
u(0) \in \mathcal{X}^{(2)}
\end{array} \quad \text { with } \quad \mathbf{R}(u, \mu)=\left[\begin{array}{c}
\mathbf{L}_{1}(\mu)\left(\mathbf{G}\left(\pi_{2}(u)\right)+\left(\mathbf{L}(\mu)-\mathbf{L}\left(\mu_{c}\right)\right) \pi_{2}(u)\right. \\
0
\end{array}\right]\right.
$$

and $\mathbf{A}$ is given by (16). It follows that $\mathbf{R}\left(0, \mu_{c}\right)=0, D \mathbf{R}\left(0, \mu_{c}\right)=0$. Indeed, we have removed the linear terms in the definition of $\mathbf{R}$. This is the Cauchy problem to which we will apply the Center Manifold Theorem. In order to apply this theorem, we need more regularity for $\mathbf{R}$. This is obtained by restricting to the spaces $\mathcal{X}^{(q)}$.

Definition 4.1 We write $\mathbf{A}_{(q)}$ the restriction of $\mathbf{A}$, defined in (16), to $\mathcal{X}^{(q)}$ with domain $D\left(\mathbf{A}_{(q)}\right)=$ $\mathcal{Z}^{(q)}$. 
Then, the regularity of the nonlinear term $\mathbf{R}$ is given by the following lemma:

Lemma 4.2 If $\mathbf{J} \in \mathrm{L}^{\infty}\left(\Omega^{2}, \mathbb{R}^{\mathrm{p} \times \mathrm{p}}\right)$ and $\mathbf{S} \in \mathcal{C}^{k}\left(\mathbb{R}^{p}, \mathbb{R}^{p}\right), k \geq q$, then for all integer $2 \leq q<\infty$,

$$
\begin{aligned}
\mathbf{A}_{(q)} & \in \mathcal{L}\left(\mathcal{Z}^{(q)}, \mathcal{X}^{(q)}\right) \\
\mathbf{R} & \in \mathcal{C}^{q-1}\left(\mathcal{Z}^{(q)} \times \mathbb{R}^{m_{p a r}}, \mathcal{Y}^{(q)}\right)
\end{aligned}
$$

and

$$
D_{u}^{q} \mathbf{R}\left(u_{0}, \mu\right)\left[u_{1}, \cdots, u_{q}\right]=\left[\begin{array}{c}
\mathbf{L}_{1}(\mu) \mathbf{S}^{(q)}\left(\mathbf{V}^{f}\right) \pi_{2}\left(u_{1} \cdots u_{q}\right) \\
0
\end{array}\right], \text { for } u_{0}=\left[\begin{array}{c}
\mathbf{V}^{f} \\
\mathbf{V}^{f}
\end{array}\right]
$$

where $u_{1} \cdots u_{p}$ is the component-wise product of the functions $u_{i}$.

Proof.

Case of $\mathbf{A}_{(q)}$. We use the continuous embeddings $W^{1, q}\left(-\tau_{m}, 0 ; \mathrm{L}^{\mathrm{q}}\left(\Omega, \mathbb{R}^{\mathrm{p}}\right) \hookrightarrow \mathrm{C}^{0}\left(-\tau_{\mathrm{m}}, 0 ; \mathrm{L}^{\mathrm{q}}\left(\Omega, \mathbb{R}^{\mathrm{p}}\right)\right)\right.$ and $\mathrm{L}^{\mathrm{q}} \hookrightarrow \mathrm{L}^{1}$. From lemma C.1:

$$
\begin{aligned}
\left\|\mathbf{L}_{1} \pi_{2}(u)\right\|_{\mathrm{L}^{\mathrm{q}}} & =O\left(\left\|\pi_{2}(u)\right\|_{C^{0}\left(-\tau_{m}, 0 ; \mathrm{L}^{1}\right)}\right) \\
& =O\left(\left\|\pi_{2}(u)\right\|_{\mathrm{W}^{1, \mathrm{q}}\left(-\tau_{\mathrm{m}}, 0 ; \mathrm{L}^{1}\right)}\right)=O\left(\left\|\pi_{2}(u)\right\|_{\mathrm{W}^{1, \mathrm{q}}\left(-\tau_{\mathrm{m}}, 0 ; \mathrm{L}^{\mathrm{q}}\right)}\right)=O\left(\|u\|_{\left.\mathcal{Z}^{(q)}\right)}\right) .
\end{aligned}
$$

It follows that $\left\|\mathbf{A}_{(q)} u\right\|_{\mathcal{X}^{(q)}}=O\left(\|u\|_{\mathcal{Z}^{(q)}}\right)$ which proves that $\mathbf{A}_{(q)}$ is continuous.

Case of $\mathbf{R}$. Let us ignore for simplicity the dependence on the parameter $\mu$. It is easy to see from the definition (28) of $\mathbf{R}$ that, if it exists, $\pi_{1} D^{q} \mathbf{R}\left(u_{0}\right)\left[u_{1}, \cdots, u_{q}\right]=\mathbf{L}_{1} \mathbf{S}^{(q)}\left(\mathbf{V}^{f}\right) \pi_{2}\left(u_{1} \cdots u_{q}\right)$ while $\pi_{2} D^{q} \mathbf{R}\left(u_{0}\right)=0$. The notation $u_{1} \cdots u_{q}$ is the component-wise product of the $q$ functions $u_{1}, \cdots, u_{q}$ in $\mathcal{Z}^{(q)}$.

We write $\phi_{i} \equiv \pi_{2} u_{i}$. As $W^{1, q}\left(-\tau_{m}, 0 ; \mathrm{L}^{\mathrm{q}}\right) \subset \mathrm{C}^{0}\left(-\tau_{\mathrm{m}}, 0 ; \mathrm{L}^{1}\right)$, we find, from the generalized Hölder's inequality, that $\forall \theta \phi_{1}(\theta) \cdots \phi_{q}(\theta)$ is in $\mathrm{L}^{1}$ and

$$
\left\|\phi_{1} \cdots \phi_{q}\right\|_{C^{0}\left(-\tau_{m}, 0 ; \mathrm{L}^{1}\right)} \leq \prod_{i=1}^{q}\left\|\phi_{i}\right\|_{C^{0}\left(-\tau_{m}, 0 ; \mathrm{L}^{\mathrm{q}}\right)}
$$

In order to prove that $\mathbf{R} \in C^{q ? 1}\left(\mathcal{Z}^{(q)} \times \mathbb{R}^{p a r}, \mathcal{Y}^{(q)}\right)$ for a given $q$, we have to prove that $D^{l} \mathbf{R}\left(u_{0}\right):\left(\mathcal{Z}^{(q)}\right)^{l} \rightarrow \mathcal{Y}^{(q)}$ for all $1 \leq l \leq q-1$ and that the remainder is small enough. Using lemma C.1, we prove the general estimate $\forall l \leq q$ :

$\left\|\mathbf{L}_{1}\left(\phi_{1} \cdots \phi_{l}\right)\right\|_{\mathrm{L}^{q}}=O\left(\left\|\left(\phi_{1} \cdots \phi_{l}\right)\right\|_{C^{0}\left(-\tau_{m}, 0 ; \mathrm{L}^{1}\right)}\right)=O\left(\prod_{i=1}^{l}\left\|\phi_{i}\right\|_{C^{0}\left(-\tau_{m}, 0 ; \mathrm{L}^{l}\right)}\right)=O\left(\prod_{i=1}^{l}\left\|\phi_{i}\right\|_{C^{0}\left(-\tau_{m}, 0 ; \mathrm{L}^{q}\right)}\right)=$ $O\left(\prod_{i=1}^{l}\left\|\phi_{i}\right\|_{W^{1, q}\left(-\tau_{m}, 0 ; \mathrm{L}^{q}\right)}\right)=O\left(\prod_{i=1}^{l}\left\|u_{i}\right\|_{\mathcal{Z}^{(q)}}\right)$. It follows that $D^{l} \mathbf{R}\left(u_{0}\right)$ for all $l \leq q-1$ exists is continuous. Using the Taylor expansion with integral remainder at order $l \leq q-1$ of $\mathbf{S}$, it is easy to find a power expansion of $\pi_{1} \mathbf{R}(u)$ up to order $l$ with remainder

$$
\frac{1}{l !} \mathbf{L}_{1}(\mu) \cdot \int_{0}^{1}(1-s)^{l} \mathbf{S}^{(l+1)}\left(\mathbf{V}^{f}+s \mathbf{U}_{t}\right) \mathbf{U}_{t}^{l+1} d s
$$

Using, the previous estimation, this remainder is of order $\|u\|_{\mathcal{Z}^{(q)}}^{l+1}, \forall l \leq q-1$ and we can conclude that $\mathbf{R}$ is $\mathcal{C}^{l}, \forall l \leq q-1$. 
Hence we have the quasi-linear formulation (26) of the nonlinear problem (4), where:

$$
\left\{\begin{array}{l}
\mathbf{A} \in \mathcal{L}\left(\mathcal{Z}^{(q)}, \mathcal{X}^{(q)}\right) \text { continuous } \\
\mathbf{R}(\cdot, \mu) \in C^{q-1}\left(\mathcal{Z}^{(q)}, \mathcal{Y}^{(q)}\right), \forall q \geq 2 \\
\mathcal{Y}^{(q)}=\mathcal{X}^{(q)}
\end{array}\right.
$$

with continuous embeddings: $\mathcal{Z}^{(q)} \hookrightarrow \mathcal{Y}^{(q)} \hookrightarrow \mathcal{X}^{(q)}$.

It easy to see that $\Sigma\left(\mathbf{A}_{(q)}\right)=\Sigma(\mathbf{A})$ if $\mathbf{J} \in \mathrm{L}^{\infty}\left(\Omega^{2}, \mathbb{R}^{\mathrm{p} \times \mathrm{p}}\right)$ and that the eigenvectors are the same for the two operators. Also $P_{\lambda}$ commutes with $\mathbf{A}_{(q)}$ because it commutes with $\mathbf{A}\left(\stackrel{\text { def }}{=} \mathbf{A}_{(2)}\right)$. As a consequence, $P_{\lambda}$ is the spectral projector of all the operators $\mathbf{A}_{(q)}$.

Lemma B.1 shows that $\mathbf{L}_{1} \in \mathcal{L}\left(\mathrm{W}^{1, \mathrm{q}}\left(-\tau_{\mathrm{m}}, 0 ; \mathrm{L}^{\mathrm{q}}\right), \mathrm{L}^{\mathrm{q}}\right)$ is continuous and [5][theorem 3.23] shows that $\mathbf{A}_{(q)}$ generates a strongly continuous semigroup $\left(\mathbf{T}_{(q)}(t)\right)$ on $\mathcal{X}^{(q)}$. Lemma B.2 and [5][proposition 4.3] imply that $\left(\mathbf{T}_{(q)}(t)\right)$ satisfies the spectral mapping theorem and that the bounds for the hyperbolic projections found in section 3.4 in the case $q=2$ are true for all integer $2 \leq q<\infty$.

From now on we drop the index ${ }^{(q)}$ for simplicity even if all the spaces depend on the integer $q$.

\subsection{Solution of the inhomogeneous problem}

Here, we prove the last condition in proposition 4.3 for the application of the center manifold theorem. This proposition is not easy to prove in general and the proof is usually done using the sufficient conditions (as given in [25] for example) i.e. the norm of the resolvent $(i \omega-\mathbf{A})^{-1}$ has to be bounded by some power of $\frac{1}{|\omega|}$. This implies that the spectrum of $\mathbf{A}$ is included in a cone centered on the real axis, an operator satisfying this condition are called sectorial. The spectrum does not satisfy this property in the general case. Indeed, from [43], we see that the spectrum is rather included in a cone with exponential boundary. Hence the resolvent $(i \omega-\mathbf{A})^{-1}$ is not bounded by some power of $\frac{1}{|\omega|}$ in our case. This is why we have to solve (27) directly.

Notice that the authors in [29] also solve (27) for 'advance-delay' scalar differential equations with a method that is different from the one we are about to describe. Let us comment a bit about the difficulties raised in solving (27). We start with a definition of a special set of functions having exponential growth:

$$
C_{\eta}^{0}(\mathbb{R}, \mathcal{E}) \equiv\left\{\phi \in C^{0}(\mathbb{R}, \mathcal{E}),\|\phi\|_{C_{\eta}^{0}} \equiv \sup _{t \in \mathbb{R}} e^{-\eta|t|}\|\phi(t)\|_{\mathcal{E}}<\infty\right\} .
$$

Let us define the hyperbolic projection of $\mathcal{Y}, \mathcal{Z}$ by $\mathcal{Y}_{h} \equiv P_{h} \mathcal{Y}, \mathcal{Z}_{h} \equiv P_{h} \mathcal{Z}$. We have to build a solution of $(27)$ in $C_{\eta}^{0}\left(\mathbb{R}, \mathcal{Z}_{h}\right)$ which is linearly and continuously depending on $F \in C_{\eta}^{0}\left(\mathbb{R}, \mathcal{Y}_{h}\right)$. This solution $u=\mathbf{K} \cdot F$ is built using a variation-of-constants formula based on a convolution of the semigroup $\mathbf{T}$ with $F$. The linear operator $\mathbf{K}$ is the operator that gives the unique solution of (27) given the term $F$. There are two main things to prove:

- $u(t) \in \mathcal{Z}_{h}$ with $F(t) \in \mathcal{Y}_{h}$.

- $\|u(t)\|_{\mathcal{Z}}$ is exponentially bounded on $\mathbb{R}$. 
However, from the proof of the center manifold theorem in [25], it can be noted that the linear operator $\mathbf{K}$ is always applied to vectors such as $P_{h} \mathbf{R}(v), P_{h} D \mathbf{R}(v) \cdots$ for some vector $v$. Given the particular shape of these vectors ${ }^{2} P_{h}\left[\begin{array}{l}\star \\ 0\end{array}\right]$, we only have to solve (32) for functions $F$ that belong to a distinct subspace of $\mathcal{Y}$, i.e. the space $P_{h}\left(\mathrm{~L}^{\mathrm{q}} \times\{0\}\right)$. This is done in the next proposition.

Proposition 4.3 Define for all integer $2 \leq q<\infty, \mathcal{Y}_{h}=P_{h} \mathcal{Y}, \mathcal{Z}_{h}=P_{h} \mathcal{Z}$. For any $\eta \in[0, \gamma]$ and for any function $F=P_{h}\left[\begin{array}{l}f \\ 0\end{array}\right] \in C_{\eta}^{0}\left(\mathbb{R}, \mathcal{Y}_{h}\right)$, the problem

$$
\dot{u}=\mathbf{A} u+F(t)
$$

has a unique solution $u=\mathbf{K}_{h} F \in C_{\eta}^{0}\left(\mathbb{R}, \mathcal{Z}_{h}\right)$ and

$$
\left\|\left|\mathbf{K}_{h}\right|\right\|_{\mathcal{L}\left(C_{\eta}^{0}\left(\mathbb{R}, \mathcal{Y}_{h}\right), C_{\eta}^{0}\left(\mathbb{R}, \mathcal{Z}_{h}\right)\right)} \leq C(\eta)
$$

with $C \in C^{0}([0, \gamma], \mathbb{R})$.

Proof. Note that there is no initial condition in (32) because the solution is required to be defined on $\mathbb{R}$ for $f$ given.

Uniqueness. If $f \equiv 0$ then any solution is given by $u(t)=\mathbf{T}(t) u(0)$ where $u(0) \in \mathcal{Z}_{h}$. To ensure that $\|u(t)\|_{\mathcal{X}}=O\left(e^{\eta|t|}\right)$ as $t \rightarrow \infty$ requires that $u(0) \in \mathcal{Z}_{s}$ but in this case $e^{-\eta|t|}\|u(t)\|_{\mathcal{X}}$ is unbounded as $t \rightarrow-\infty$ unless $u(0)=0$. Hence the only solution when $f=0$ is $u_{h}=0$. This proves uniqueness of the solution.

Existence. Write

$$
\left(\mathbf{K}_{h} F\right)(t) \equiv \int_{-\infty}^{t} \mathbf{T}_{s}(t-r) F(r) d r-\int_{t}^{\infty} \mathbf{T}_{u}(t-r) F(r) d r
$$

The second term $u_{u}(t) \equiv-\int_{t}^{\infty} \mathbf{T}_{u}(t-r) F(r) d r$ will not be considered in this proof. Indeed, as $\mathbf{T}_{u}(t)$ has a finite dimensional range, it is straightforward to prove that $u_{u}(t)$ fulfills all the properties stated in the proposition. Rather, we will focus on the study of $u_{s}(t) \equiv \int_{-\infty}^{t} \mathbf{T}_{u}(t-$ $r) F(r) d r$ because it is more difficult to show that it yields a solution of the inhomogeneous problem.

The first difficulty is to prove that $u_{s}(t) \in \mathcal{Z}$, i.e. the convolution of $F$ by $\mathbf{T}_{s}$ yields a vector in the domain of $\mathbf{A}$. This is done in three steps. First, we study in lemma C.2 the general properties of $\mathbf{T}(t)\left[\begin{array}{l}x \\ 0\end{array}\right]$. Then, we use a variation-of-constants formula in lemma C.2 to compute $\mathbf{T}(t)$ by a perturbation result. Finally, proposition C.4 shows that:

- $u_{s} \in C_{\eta}^{0}(\mathbb{R}, \mathcal{Z})$ and $\left\|u_{s}\right\|_{C_{\eta}^{0}(\mathbb{R}, \mathcal{Z})} \leq K(\eta)\|f\|_{C_{\eta}^{0}(\mathbb{R}, \mathcal{Y})}$ with $\eta \rightarrow K(\eta)$ positive continuous,

- $u_{s} \in C^{1}(\mathbb{R}, \mathcal{X})$,

- $u_{s}$ satisfies $\dot{u}_{s}=\mathbf{A} u_{s}+P_{s}\left[\begin{array}{l}f \\ 0\end{array}\right]$ on $\mathcal{X}$, i.e. is a classical solution.

This concludes the proof of the proposition.

\footnotetext{
${ }^{2}$ see the definition of $\mathbf{R}$ in (30).
} 


\subsection{Center manifold and reduced equation}

We are now in a position to state the center manifold manifold for an integer $q$ sufficiently large to ensure the regularity of $\mathbf{R}$ required by a series expansion for example. Note again that we drop the index ${ }^{(q)}$ in the names of the different spaces.

Theorem 4.4 (Center manifold for delayed neural fields equations) Let us write $\mathcal{X}_{c}$ the vector space of generalized eigenvectors of $\mathbf{A}$ with zero real part. As it is finite dimensional, we can write $\mathcal{X}=\mathcal{X}_{c} \oplus \mathcal{X}_{h}$ where $\mathcal{X}_{h}$ is the hyperbolic part of the history space. Then, there exist a neighbourhood $\mathcal{O}=\mathcal{O}_{u} \times \mathcal{O}_{\mu}$ of $(0,0)$ in $\mathcal{X} \times \mathbb{R}^{m_{\text {par }}}$, a mapping $\Psi \in C^{q}\left(\mathcal{X}_{c} \times \mathbb{R}^{m_{\text {par }}} ; \mathcal{Z}_{h}\right)$ with $\Psi(0,0)=0, \quad D \Psi(0,0)=0$ and a manifold $\mathcal{M}(\mu)=\left\{u_{c}+\Psi\left(u_{c}, \mu\right), u_{c} \in \mathcal{X}_{c}\right\}$ for $\mu \in \mathcal{O}_{\mu}$ such that:

1. $\mathcal{M}(\mu)$ is locally invariant, i.e., if $u$ is a solution of (30) satisfying $u(0) \in \mathcal{M}(\mu) \cap \mathcal{O}_{u}$ and $u(t) \in \mathcal{O}_{u}$ for all $t \in[0, T]$, then $u(t) \in \mathcal{M}(\mu)$ for all $t \in[0, T]$.

2. $\mathcal{M}(\mu)$ contains the set of bounded solutions of (30) staying in $\mathcal{O}_{u}$ for all $t \in \mathbb{R}$, i.e. if $u$ is a solution of (30) satisfying for all $t \in \mathbb{R}, u(t) \in \mathcal{O}_{u}$, then $u(0) \in \mathcal{M}(\mu)$.

3. (Parabolic case) if $\Sigma_{u}(\mathbf{A})=\emptyset$, then $\mathcal{M}(\mu)$ is locally attracting, i.e. if $u$ is a solution of (30) satisfying $u(0) \in \mathcal{O}_{u}$ and $u(t) \in \mathcal{O}_{u}$ for all $t>0$, then there exists $v(0) \in \mathcal{M}(\mu) \cap \mathcal{O}_{u}$ and $\tilde{\gamma}>0$ such that

$$
u(t)=v(t)+O\left(e^{-\tilde{\gamma} t}\right) \text { as } t \rightarrow \infty
$$

where $v$ is a solution of (30) with initial condition $v(0)$.

Proof.

1-2 Having written our nonlinear problem as (31), in order to apply [25, theorem 2.9] we have to check several hypotheses. The first is to check the existence of a spectral decomposition with positive spectral gap $\gamma$ where the central part $\left.\mathbf{A}_{c} \equiv \mathbf{A}\right|_{\mathcal{X}_{c}}$ has only a finite number of eigenvalues with finite algebraic multiplicities, this was proved in section 3.4 (see also the end of section 4.1). Then, we have to check an hypothesis regarding the existence of solutions with exponential divergence at $t= \pm \infty$ : this was done in proposition 4.3. As a consequence, we can apply [25, Theorem 2.9] and obtain the theorem.

3 This is a consequence of [25, theorem 3.23] which requires several conditions to be checked. These conditions are very similar to the ones for 1-2. They are given and checked in appendix C.3.

From [25, Corollary 2.12], consider a solution $u$ of (30) which belongs to $\mathcal{M}(\mu)$ for $t \in \mathbb{R}$, then $u=u_{c}+\Psi\left(u_{c}, \mu\right)$ with $u_{c} \in \mathcal{X}_{c}$ and $u_{c}$ satisfies

$$
\frac{d u_{c}}{d t}=\mathbf{A} u_{c}+P_{c} \mathbf{R}\left(u_{c}+\Psi\left(u_{c}, \mu\right), \mu\right)
$$

where the projector $P_{c}$ is defined by the Dunford formula. It is known (see [31, theorem III.6.17]) that the projector given by the Dunford formula is the unique spectral projector that commutes with A. Hence, we have the expression from section 3.4, $P_{c}=\sum_{\lambda \in \Sigma_{c}(\mathbf{A})} P_{\lambda}$ with $P_{\lambda}$ given in proposition 3.8 .

Let us derive a simpler equation for $u_{c}$. Write $u_{c}=\sum_{i=1}^{\operatorname{dim} \mathcal{X}_{c}} z_{i} \phi_{i}$ where $z_{i}$ are complex numbers and $\phi_{i}, i=1, \cdots, \operatorname{dim} \mathcal{X}_{c}$ is a basis of $\mathcal{X}_{c}$ (see proposition 3.4). We want to write ordinary 
differential equations for the coordinates $z_{i}$. As $\mathcal{X}_{c}$ is invariant by $\mathbf{A}$, there is a matrix $A_{c}$ of size $\operatorname{dim} \mathcal{X}_{c}$ such that: $\mathbf{A} u_{c}=\sum_{i=1}^{\operatorname{dim} \mathcal{X}_{c}}\left(A_{c} z\right)_{i} \phi_{i}$ with $z=\left(z_{1}, \cdots, z_{\operatorname{dim} \mathcal{X}_{c}}\right)$. To find equations for the $z_{i}$, we need to project (33) on each generalized eigenvector $\phi_{i}$. Hence, let us consider a family of vectors $\psi_{i}$ as in proposition 3.8, then $\left\langle\left\langle\psi_{i}, u_{c}\right\rangle\right\rangle=z_{i}$ and $\left\langle\left\langle\psi_{i}, \mathbf{R}\left(u_{c}+\Psi\left(u_{c}, \mu\right), \mu\right)\right\rangle\right\rangle=$ $\left\langle\pi_{1} \psi_{i}, \pi_{1} \mathbf{R}\left(u_{c}+\Psi\left(u_{c}, \mu\right), \mu\right)\right\rangle_{\mathrm{L}^{2}}$ (see proposition 3.8 for the bilinear product). We use these expressions together with (33) to obtain the reduced equations:

$$
\dot{z}_{i}=\left(A_{c} z\right)_{i}+\left\langle\pi_{1} \psi_{i}, \pi_{1} \mathbf{R}\left(u_{c}+\Psi\left(u_{c}, \mu\right), \mu\right)\right\rangle_{L^{2}}
$$

This equation was given in [24, 22, 47] under different hypotheses.

It should be noted that most of the neural fields models are used close to a stationary bifurcation (see for example $[6,9]$ ). This bifurcation can be changed by the introduction of the delays producing either a purely imaginary eigenvalue or changing the algebraic multiplicity of the static eigenvalue. No general criterion is known for the appearance of a purely imaginary eigenvalue (but see [43]). However such a criterion exists for the algebraic multiplicity and allows to test very easily if a Bogdanov-Takens can emerge from the initial static bifurcation. This is done in the next lemma:

Lemma 4.5 Let us consider a stationary cortical state $\mathbf{V}^{f}$ of (4) and the (time) constant function $\phi=\left[\begin{array}{l}e_{1} \\ e_{1}\end{array}\right]$ in the one-dimensional kernel of $\mathbf{A}$ where $e_{1} \in \mathrm{L}^{2}$. We write $\psi=\left[\begin{array}{c}e_{1}^{*} \\ e_{1}^{*}\end{array}\right]$ the constant vector in ker $\mathbf{A}^{*}$ with $\left\langle e_{1}^{*}, e_{1}\right\rangle_{\mathrm{L}^{2}}=1$. The algebraic multiplicity of the zero eigenvalue is at least two if and only if

$$
0=\langle\langle\psi, \phi\rangle\rangle=1+\left\langle e_{1}^{*}, \tilde{\mathbf{J}} \tau e_{1}\right\rangle_{\mathrm{L}^{2}}
$$

Proof. We use proposition 3.4) to express ker $\mathbf{A}^{2}$ :

$$
\text { ker } \mathbf{A}^{2}=\{V+\theta U,(U, V) \text { is a Jordan chain for } \Delta(0)\}
$$

is two-dimensional. By the lemma A.12, we have $\Delta(0) U=0, \Delta^{\prime}(0) U+\Delta(0) V=0$ which gives $\left[\begin{array}{l}U \\ U\end{array}\right] \in$ ker $\mathbf{A}$ and $U=e_{1}$. From the Fredholm alternative, ker $\mathbf{A}^{2}$ is larger than ker $\mathbf{A}$ if and only if $\left\langle e_{1}^{*}, \Delta^{\prime}(0) e_{1}\right\rangle_{\mathrm{L}^{2}}=0$. From (17): $\Delta^{\prime}(0)=\mathrm{Id}+\tilde{\mathbf{J}} \boldsymbol{\tau}$. Therefore $\left\langle e_{1}^{*}, \Delta^{\prime}(0) e_{1}\right\rangle_{\mathrm{L}^{2}}=1+\left\langle e_{1}^{*}, \tilde{\mathbf{J}} \boldsymbol{\tau} e_{1}\right\rangle_{\mathrm{L}^{2}}$. Notice that this last quantity is equal to $\langle\langle\psi, \phi\rangle\rangle$. The lemma is proved.

\subsection{Normal form of the Pitchfork bifurcation}

As we have mentioned earlier, many of the neural fields models operate near a static bifurcation point (see for example [6,9]), it is thus interesting to see how it is altered by the introduction of delays. We will treat the case of the pitchfork bifurcation (see $[32,25]$ ), the case of the transcritical bifurcation is very similar. We look at the quantitative modification of the reduced equation (in the non-delayed case) from the introduction of delays. Recall that we consider an equilibrium $\mathbf{V}^{f}$ and that we write an equation for $\mathbf{U}=\mathbf{V}-\mathbf{V}^{f}$. Let us consider the nonlinear gain $\sigma \in \mathbb{R}$ as a bifurcation parameter. Suppose that $e_{1}$ (resp. $e_{1}^{*}$ ) is in the one-dimensional kernel of $\Delta(0)=-\mathbf{L}_{0}+\mathbf{J}(0)$ (resp. $\left.\Delta(0)^{*}=-\mathbf{L}_{0}+\mathbf{J}(0)^{*}\right)$ at $\sigma=\sigma_{c}$. Then the reduced equation for the non-delayed neural mass equation near $\sigma=\sigma_{c}$ with $\mathbf{V}=\mathbf{V}^{f}+x e_{1}+o(x)$ reads (see [45]):

$$
\dot{x}=\frac{\sigma-\sigma_{c}}{\sigma_{c}} x+\chi_{q} x^{q}+o\left(x^{q}\right)
$$

$\mathrm{RR} \mathrm{n}^{\circ} 8020$ 
for some $\chi_{q} \neq 0$. What happens if we introduce space-dependent delays? If the delays are small, we expect the eigenvalues of $\mathbf{A}$ to be close to the spectrum of the non-delayed linearized equation, thus yielding again a Pitchfork or a Transcritical bifurcation. Notice that $e_{1}$ is in the kernel of $\Delta(0)$, hence $0 \in \Sigma_{p}(\mathbf{A})$. Let us write $\phi=\left[\begin{array}{l}e_{1} \\ e_{1}\end{array}\right] \in \operatorname{ker} \mathbf{A}$, from proposition 3.8, we find that $\psi=\beta\left[\begin{array}{l}e_{1}^{*} \\ e_{1}^{*}\end{array}\right] \in \operatorname{ker} \mathbf{A}^{*}$ with $\beta \in \mathbb{R}$. It is normalized such that $\langle\langle\psi, \phi\rangle\rangle=1$. Some algebra shows that $\langle\langle\psi, \phi\rangle\rangle=\beta+\beta\left\langle\mathbf{J} D \mathbf{S} \boldsymbol{\tau} e_{1}, e_{1}^{*}\right\rangle_{\mathrm{L}^{2}}$ where $\mathbf{J} D \mathbf{S} \boldsymbol{\tau}$ is the integral operator on $\mathcal{F}$ with kernel ${ }^{3}$ $\mathbf{J}\left(\mathbf{r}, \mathbf{r}^{\prime}\right) D S\left(\mathbf{V}^{f}\left(\mathbf{r}^{\prime}\right)\right) \boldsymbol{\tau}\left(\mathbf{r}, \mathbf{r}^{\prime}\right)$. The normalization condition requires:

$$
1+\left\langle\mathbf{J} D \mathbf{S} \tau e_{1}, e_{1}^{*}\right\rangle_{\mathrm{L}^{2}} \neq 0
$$

When this is true, we define $\beta^{-1} \equiv 1+\left\langle\mathbf{J} D \mathbf{S} \boldsymbol{\tau} e_{1}, e_{1}^{*}\right\rangle_{\mathrm{L}^{2}}$. Note that the above condiiton is equivalent to saying that 0 is a simple eigenvalue of $\mathbf{A}$ (see [24, 20] and lemma 4.5). Then $u_{c}=x \phi+\Psi(x \phi, \sigma)$ and $A_{c}=0$ because $\mathbf{A} \phi=0$. Hence, the reduced equation (34) reads:

$$
\dot{x}=\left\langle\pi_{1} \psi, \pi_{1} \mathbf{R}\left(u_{c}+\Psi\left(u_{c}, \sigma\right), \sigma\right)\right\rangle_{\mathrm{L}^{2}}=\beta\left\langle e_{1}^{*}, \pi_{1} \mathbf{R}\left(u_{c}+\Psi\left(u_{c}, \sigma\right), \sigma\right)\right\rangle_{\mathrm{L}^{2}}
$$

From theorem 4.4, $\Psi\left(u_{c}, \sigma\right)=o(|x|)$ which gives $\mathbf{R}\left(u_{c}+\Psi\left(u_{c}, \sigma\right), \sigma\right)=\mathbf{R}\left(u_{c}, \sigma\right)+$ h.o.t.. By using (30), the reduced equation (35) is now:

$$
\begin{aligned}
\dot{x} / \beta & =\left\langle e_{1}^{*}, \pi_{1} \mathbf{R}\left(u_{c}, \sigma\right)\right\rangle_{\mathrm{L}^{2}}+\text { h.o.t. }=\frac{\sigma-\sigma_{c}}{\sigma_{c}} x+\left\langle e_{1}^{*}, G\left(x e_{1}\right)\right\rangle_{\mathrm{L}^{2}}+\text { h.o.t. } \\
& =\frac{\sigma-\sigma_{c}}{\sigma_{c}} x+\chi_{q} x^{q}+o\left(x^{q}\right),
\end{aligned}
$$

from the definition of $\chi_{q}$ in [45] and (29). Thus, introducing delays only results in a rescaling of time. This analysis holds as long as the only eigenvalue at $\sigma_{c}$ is the simple 0 eigenvalue. Notice that another eigenvalue may approach the imaginary axis by increasing the delays (decreasing the propagation speed), if it is purely imaginary, it would lead to a Fold-Hopf bifurcation scenario, if it is 0 , it would lead to a Bogdanov-Takens bifurcation scenario.

\section{Conclusion}

In this article, we have continued our efforts for developing a theoretical framework for the study of neural fields equations with space dependent delays. In [45] we proved the existence, uniqueness and the boundedness of the solutions to these equations for continuous history segments in $C^{0}\left(-\tau_{m}, 0 ; \mathrm{L}^{2}\right)$. We also proved that the stationary states of $(1)$ are stable in $C^{0}\left(-\tau_{m}, 0 ; \mathrm{L}^{2}\right)$ if and only if the punctual spectrum of the linearized operator is in the left part of the complex plane. This was only a start in the direction of understanding the delayed neural fields equations.

By using the Hilbert space $\mathcal{X}^{(2)}$ for the history space and combining ideas from [24] and [5], we have been able to find a closed form formula for the spectral projector. This formula leads naturally to the introduction of the bilinear product, a quantity introduced in [24] which is difficult to generalize from functional differential equations with values in $\mathbb{R}^{n}$ to equations with values in a Banach space (see [1]).

We then proved a center manifold theorem for the delayed neural fields equations. This was not an easy task as the usual estimate tools for sectorial operators do no apply to this case. We used the more powerful tools of the semigroups theory as exposed in $[18,5]$. Hence we combined ideas from [25] and [18, 5] to prove a center manifold theorem. This being achieved, we could apply a "generic" normal form theory as in [25] in order to obtain the main normal forms. It

\footnotetext{
${ }^{3}$ It is a component-wise product.
} 
turned out that the computation of normal forms for the delayed neural fields equations is no more difficult than for the non-delayed equations.

Our ultimate goal is to understand how the delays qualitatively shape the dynamics of the solutions. Hence, after the proof of the center manifold theorem, we explored how a Pitchfork bifurcation is altered by the introduction of delays. This is important because most of the neural fields models operate near such a stationary bifurcation. The conclusion is that the Pitchfork is unaltered (it is only scaled in time) if the delays are smaller than a given bound. For larger delays, the Pitchfork bifurcation may degenerate into a Bogdanov-Takens bifurcation or a FoldHopf bifurcation.

This paper calls for more work concerning neural fields equations with space dependent delays and some preliminary work seems to imply the existence of a rich dynamics where multiple bifurcations with symmetries interact with global orbits.

\section{Acknowledgement}

Thes authors would like to thank G.Iooss for his valuable comments and criticisms on this manuscript.

\section{A Operators and their spectra}

We recall and gather in this appendix a number of definitions, results and hypotheses that are used in the body of the article to make it more self-sufficient.

Definition A.1 We note $\||\mathbf{J}|\|_{\mathrm{L}^{2}}$ the operator norm of a bounded operator $\mathbf{J} \in \mathcal{L}\left(\mathrm{L}^{2}, \mathrm{~L}^{2}\right)$, ie

$$
\sup _{\|\mathbf{V}\|_{L^{2}} \leq 1} \frac{\|\mathbf{J} \cdot \mathbf{V}\|_{L^{2}}}{\|\mathbf{V}\|_{L^{2}}}
$$

It is known, see e.g. [31], that

$$
\||\mathbf{J}|\|_{L^{2}} \leq\|\mathbf{J}\|_{L^{2}\left(\Omega^{2}, \mathbf{R}^{\mathrm{p} \times \mathrm{p}}\right)}
$$

Definition A.2 A semigroup $(\mathbf{T}(t))_{t \geq 0}$ on a Banach space $X$ is strongly continuous if $\forall x \in X$, $t \rightarrow T(t) x$ is continuous from $\mathbb{R}_{+}$to $\bar{X}$.

Definition A.3 A semigroup $(T(t))_{t \geq 0}$ on a Banach space $X$ is norm continuous if $t \rightarrow T(t)$ is continuous from $\mathbb{R}_{+}$to $L(X)$. It is said eventually norm continuous if $t \rightarrow \mathbf{T}(t)$ is norm continuous from $\left(t_{0}, \infty\right)$ to $\mathcal{L}(X)$. If $t_{0}$ can be chosen to be 0 , we say that $(T(t))_{t \geq 0}$ is immediately norm continuous.

Theorem A.4 Let $(T(t))_{t \geq 0}$ be a strongly continuous semigroup on a Banach space $X$ with generator $(\mathbf{A}, D(\mathbf{A}))$. Moreover, assume that $(T(t))_{t \geq 0}$ is eventually norm continuous. Then the Spectral Mapping Theorem

$$
\Sigma(T(t)) \backslash\{0\}=e^{t \Sigma(\mathbf{A})}
$$

$\mathrm{RR} \mathrm{n}^{\circ} 8020$ 
holds for all $t \geq 0$. As a consequence, if $\sup \Re \Sigma(\mathbf{A})<0$, then there exists $M \geq 1$ such that:

$$
\||| T(t)\| \mid \leq M e^{t \sup \Re \Sigma(\mathbf{A}) / 2}, \quad \forall t \geq 0 .
$$

Theorem A.5 (Miyadera-Voigt,[18]) Let $(A, D(A))$ be the generator of a strongly continuous semigroup $(T(t))_{t \geq 0}$ on a Banach space $X$ and let $C \in \mathcal{L}\left(\left(D(A),\|\cdot\|_{A}\right), X\right)$ with $\|x\|_{A} \equiv\|x\|+$ $\|A x\|$. Assume that there exist constants $t_{0}>0,0 \leq q<1$ such that

$$
\int_{0}^{t_{0}}\|C T(s) x\| d s \leq q\|x\|, \forall x \in D(A)
$$

Then $(A+C, D(A))$ generates a strongly continuous semigroup $(U(t))_{t \geq 0}$ on $X$ which satisfies $\forall x \in D(A)$ :

$$
\begin{aligned}
U(t) x & =T(t) x+\int_{0}^{t} T(t-s) C U(s) x d s \\
& =T(t) x+\int_{0}^{t} U(t-s) C T(s) x d s .
\end{aligned}
$$

Theorem A.6 ([5]) Let $(A, D(A))$ be the generator of a strongly continuous semigroup $(T(t))_{t \geq 0}$ on a Banach space $X$ and let $C \in \mathcal{L}\left(\left(D(A),\|\cdot\|_{A}\right), X\right)$ with $\|x\|_{A} \equiv\|x\|+\|A x\|$. Define the abstract Volterra operator $V: C^{0}\left(\mathbb{R}_{+}, \mathcal{L}(X)\right) \rightarrow C^{0}\left(\mathbb{R}_{+}, \mathcal{L}(X)\right)$ by $V F \equiv t \rightarrow \int_{0}^{t} F(t-s) C T(s) d s$ on $D(A)$ and by its continuous extension on $X$. If the following assumptions are satisfied:

- there exist a constant $\epsilon>0$ and a function $q:[0, \epsilon) \rightarrow \mathbb{R}_{+}$such that

$$
\int_{0}^{t}\|C T(s) x\| d s \leq q(t)\|x\|, \forall x \in D(A), \forall t \in[0, \epsilon)
$$

with $\lim _{t \searrow 0} q(t)=0$.

- $(T(t))_{t \geq 0}$ is norm continuous for $t>\alpha$

- there exists $n \in \mathbb{N}$ such that $V^{n} T$ is norm continuous for $t>0$

Then $(A+C, D(A))$ generates a strongly continuous semigroup on $X$ which is norm continuous for $t>n \alpha$.

Definition A.7 A closed operator $T \in \mathcal{L}(X)$ of a Banach space $X$ is Fredholm if dim $\mathcal{N}(T)$ and $\operatorname{codim} \mathcal{R}(T)$ are finite and $\mathcal{R}(T)$ is closed in $X$.

Definition A.8 A closed operator $T \in \mathcal{L}(X)$ of a Banach space $X$ is semi-Fredholm if $\operatorname{dim} \mathcal{N}(T)$ or $\operatorname{codim} \mathcal{R}(T)$ is finite and $\mathcal{R}(T)$ is closed in $X$.

Definition A.9 If $T \in \mathcal{L}(X)$ is a closed operator of a Banach space $X$ the essential spectrum $\Sigma_{\text {ess }}(T)$ is the set of $\lambda$ s in $\mathbb{C}$ such that $\lambda \mathrm{Id}-T$ is not semi-Fredholm i.e. either $\mathcal{R}(\lambda \mathrm{Id}-T)$ is not closed or $\mathcal{R}(\lambda \mathrm{Id}-T)$ is closed but $\operatorname{dim} \mathcal{N}(\lambda \operatorname{Id}-T)=\operatorname{codim} \mathcal{R}(\lambda \operatorname{Id}-T)=\infty$. 
Definition A.10 ([24]) If $K(\lambda): \mathcal{B}_{1} \rightarrow \mathcal{B}_{2}$, the $\mathcal{B}_{i}$ being complex Banach spaces, be a linear operator-valued functions that depends analytically on $\lambda \in \mathbb{C}$. A point $\lambda_{0}$ is a characteristic value of $K$ if $\exists x_{0} \in \mathcal{B}_{1} \backslash\{0\}$ such that $K\left(\lambda_{0}\right) x_{0}=0$. An ordered set $\left(x_{0}, \cdots, x_{k-1}\right) \subset \mathcal{B}_{1}$ is a Jordan chain for $K\left(\lambda_{0}\right)$ if $x_{0} \neq 0$ and $K(z) \alpha(z)=O\left(\left(z-\lambda_{0}\right)^{k}\right)$ where $\alpha$ is the root function:

$$
\alpha(z)=\sum_{i=0}^{k-1}\left(z-\lambda_{0}\right)^{i} x_{i}
$$

The maximum length of the Jordan chains starting at $x_{0}$ is called the rank of $x_{0}$.

Definition A.11 ([24]) We call $\left(x_{1,0}, \cdots, x_{1, r_{1}-1}, \cdots, x_{p, 0}, \cdots, x_{p, r_{p}-1}\right)$ a canonical system of Jordan chains for $K\left(\lambda_{0}\right)$ if $\left(x_{1,0}, \cdots, x_{p, 0}\right)$ is a basis of $K$ er $K\left(\lambda_{0}\right)$ and for each $k,\left(x_{k, 0}, \cdots, x_{k, r_{k}-1}\right)$ is a Jordan chain of rank $r_{k}$.

Lemma A.12 ([24]) $\left(x_{0}, \cdots, x_{p-1}\right)$ is Jordan chain of length $p$ for $K\left(\lambda_{0}\right)$ if and only if $\left(x_{0}, \cdots, x_{p-1}\right) \in$ $\operatorname{Ker} K_{p}$ with

$$
K_{p}=\left[\begin{array}{cccc}
K\left(\lambda_{0}\right) & 0 & \cdots & 0 \\
\frac{d}{d z} K\left(\lambda_{0}\right) & K\left(\lambda_{0}\right) & \cdots & 0 \\
\vdots & & \ddots & \vdots \\
\frac{1}{(p-1) !} \frac{d^{p-1}}{d z^{p-1}} K\left(\lambda_{0}\right) & \frac{1}{(p-2) !} \frac{d^{p-2}}{d z^{p-2}} K\left(\lambda_{0}\right) & \cdots & K\left(\lambda_{0}\right)
\end{array}\right]
$$

Proof. Easy to prove from a Taylor expansion of $\lambda \rightarrow K(\lambda)$ at $\lambda=\lambda_{0}$.

\section{B Regularity}

Lemma B.1 If we define $\mathrm{L}^{\mathrm{q}} \equiv \mathrm{L}^{\mathrm{q}}\left(\Omega, \mathbf{R}^{\mathrm{p}}\right)$. Then, we have $\forall \phi \in W^{1, q}\left(-\tau_{m}, 0 ; \mathrm{L}^{\mathrm{q}}\right)$,

$$
\mathbf{L}_{1} \phi=\mathbf{J} \phi(0)-\int_{-\tau_{m}}^{0} \mathbf{J}[s] \dot{\phi}(s) d s
$$

where $\forall s \in\left[-\tau_{m}, 0\right], J_{i j}\left(\mathbf{r}, \mathbf{r}^{\prime}\right)[s] \equiv J_{i j}\left(\mathbf{r}, \mathbf{r}^{\prime}\right) H\left(s+\tau_{i j}\left(\mathbf{r}, \mathbf{r}^{\prime}\right)\right)$ and $H$ is the Heaviside function.

Proof.

$$
\begin{aligned}
\left(\left(\mathbf{L}_{1} \phi\right)(\mathbf{r})\right)_{i}= & \sum_{j=1}^{p} \int_{\Omega} J_{i j}(\mathbf{r}, \overline{\mathbf{r}}) \phi_{j}\left(\overline{\mathbf{r}},-\tau_{i j}(\mathbf{r}, \overline{\mathbf{r}})\right) d \overline{\mathbf{r}} \\
& =-\sum_{j=1}^{p} \int_{\Omega} J_{i j}(\mathbf{r}, \overline{\mathbf{r}})\left[\int_{-\tau_{i j}(\mathbf{r}, \overline{\mathbf{r}})}^{0} \dot{\phi}_{j}(\mathbf{r}, s) d s-\phi_{j}(\mathbf{r}, 0)\right] \\
= & \sum_{j=1}^{p} J_{i j} \phi_{j}(0)-\sum_{j=1}^{p} \int_{\Omega} J_{i j}(\mathbf{r}, \overline{\mathbf{r}}) \int_{-\tau_{m}}^{0} \dot{\phi}_{j}(\mathbf{r}, s) H\left(s+\tau_{i j}(\mathbf{r}, \overline{\mathbf{r}})\right) d s \\
& =(\mathbf{J} \phi(0))_{i}-\int_{-\tau_{m}}^{0}\left(\int_{\Omega} \mathbf{J}(\mathbf{r}, \overline{\mathbf{r}}) H(s+\boldsymbol{\tau}(\mathbf{r}, \overline{\mathbf{r}})) \dot{\phi}(\mathbf{r}, s)\right)
\end{aligned}
$$

$\operatorname{RR} n^{\circ} 8020$ 
As $\boldsymbol{\tau}$ is continuous, $\mathbf{J}\left(\mathbf{r}, \mathbf{r}^{\prime}\right)[s] \in \mathrm{L}^{\mathrm{q}}\left(\Omega^{2} \times\left[-\tau_{\mathrm{m}}, 0\right], \mathbb{R}^{\mathrm{p} \times \mathrm{p}}\right)$.

Let us define the operator:

$$
\forall \mathbf{U} \in \mathrm{L}^{\mathrm{q}}, \forall \theta \in\left[-\tau_{\mathrm{m}}, 0\right], \quad\left(\mathrm{S}_{\mathrm{t}} \mathbf{U}\right)(\theta)=\left\{\begin{array}{clr}
e^{-(t+\theta) \mathbf{L}_{0}} \mathbf{U} & \text { if } & -t<\theta \leq 0 \\
0 & \text { if } & -\tau_{m}<\theta \leq-t
\end{array}\right.
$$

and the nilpotent semigroup

$$
\forall \phi \in \mathrm{L}^{\mathrm{q}}\left(-\tau_{\mathrm{m}}, 0 ; \mathrm{L}^{\mathrm{q}}\right), \forall \theta \in\left[-\tau_{\mathrm{m}}, 0\right], \quad\left(\mathrm{T}_{0}(\mathrm{t}) \phi\right)(\theta)=\left\{\begin{array}{cl}
\phi(t+\theta) & \text { if } t+\theta \leq 0 \\
0 & \text { if } \quad t+\theta>0
\end{array}\right.
$$

Hence $T_{0}(t)=0$ if $t>\tau_{m}$. We show that the required property $(\mathrm{M})$ in [5] is true:

Lemma B.2 If $\mathbf{J} \in \mathrm{L}^{\infty}\left(\Omega^{2}, \mathbb{R}^{\mathrm{p}}\right)$, then for each space $\mathcal{X}^{(q)} \equiv \mathrm{L}^{\mathrm{q}} \times \mathrm{L}^{\mathrm{q}}\left(-\tau_{\mathrm{m}}, 0 ; \mathrm{L}^{\mathrm{q}}\right)$ where $\mathrm{L}^{\mathrm{q}} \equiv$ $\mathrm{L}^{\mathrm{q}}\left(\Omega, \mathbf{R}^{\mathrm{p}}\right)$ and $2 \leq q<\infty$, there exists $Q: \mathbb{R}_{+} \rightarrow \mathbb{R}_{+}$with $\lim _{t \rightarrow 0^{+}} Q(t)=0$ such that

$$
\forall\left[\begin{array}{l}
x \\
\phi
\end{array}\right] \in D(\mathbf{A}) \quad \int_{0}^{t}\left\|\mathbf{L}_{1}\left(S_{s} x+T_{0}(s) \phi\right)\right\|_{\mathrm{L}^{q}} d s \leq Q(t)\left\|\left[\begin{array}{l}
x \\
\phi
\end{array}\right]\right\|_{\mathcal{X}^{(q)}} .
$$

Proof. Let us first focus on the term $\int_{0}^{t}\left\|\mathbf{L}_{1}\left(S_{s} x\right)\right\|_{\mathrm{L}^{\mathrm{q}}} d s$. As $t \geq 0$, we find $\left(\mathbf{L}_{1}\left(S_{s} x\right)\right)(\mathbf{r})=$ $\int d \overline{\mathbf{r}} \mathbf{1}_{[\tau(\mathbf{r}, \overline{\mathbf{r}}), \infty)}(s) \mathbf{J}(\mathbf{r}, \overline{\mathbf{r}}) e^{-\left(s-\tau(\mathbf{r}, \overline{\mathbf{r}}) \mathbf{L}_{0}\right.} x(\overline{\mathbf{r}})$. For $s$ fixed, $\mathbf{J}_{2}(s):(\mathbf{r}, \overline{\mathbf{r}}) \rightarrow \mathbf{1}_{[\tau(\mathbf{r}, \overline{\mathbf{r}}), \infty)}(s) \mathbf{J}(\mathbf{r}, \overline{\mathbf{r}}) e^{-\left(s-\tau(\mathbf{r}, \overline{\mathbf{r}}) \mathbf{L}_{0}\right.}$ defines an integral operator on $\mathrm{L}^{\mathrm{q}}$ such that $\mathbf{L}_{1}\left(S_{s} x\right)=\mathbf{J}_{2}(s) x$. Its norm is bounded ${ }^{4}$ by: $\left\|\left|\mathbf{J}_{2}(s)\left\|\left.\right|_{L^{q}} \leq|\Omega| \sqrt{p}\right\| \mathbf{J} \|_{L^{\infty}\left(\Omega^{2}, \mathbb{R}^{p}\right)}\right.\right.$. This gives $\left.\left.\int_{0}^{t}\left\|\mathbf{L}_{1}\left(S_{s} x\right)\right\|_{\mathcal{F}} d s \leq t\right| \Omega \mid \sqrt{p}\right\| \mathbf{J} \|_{L^{\infty}\left(\Omega^{2}, \mathbb{R}^{p}\right)}$.

Let us look at the second term $\int_{0}^{t}\left\|\mathbf{L}_{1}\left(T_{0}(s) \phi\right)\right\|_{\mathrm{L}^{\mathrm{q}}} d s$. As $t \geq 0$, we find $\left(\mathbf{L}_{1}\left(T_{0}(s) \phi\right)\right)(\mathbf{r})=$ $\int d \overline{\mathbf{r}} \mathbf{1}_{[0, \tau(\mathbf{r}, \overline{\mathbf{r}})]}(s) \mathbf{J}(\mathbf{r}, \overline{\mathbf{r}}) \phi(s-\tau(\mathbf{r}, \overline{\mathbf{r}}), \overline{\mathbf{r}})$. We write $\int_{0}^{t} d s\left\|\mathbf{L}_{1}\left(T_{0}(s) \phi\right)\right\|_{\mathrm{L}^{q}}=\int_{0}^{t} h^{1 / q}$ with

$$
h(s) \equiv \int_{\Omega} d \mathbf{r}\left\|\int_{\Omega} d \overline{\mathbf{r}} \mathbf{1}_{[0, \tau(\mathbf{r}, \overline{\mathbf{r}})]}(s) \mathbf{J}(\mathbf{r}, \overline{\mathbf{r}}) \phi(s-\tau(\mathbf{r}, \overline{\mathbf{r}}), \overline{\mathbf{r}})\right\|_{\mathbb{R}^{p}}^{q} .
$$

We apply the Hölder inequality with $\bar{q}$ such that $q^{-1}+\bar{q}^{-1}=1$ :

$$
\begin{gathered}
\forall \mathbf{r},\left\|\int_{\Omega} d \overline{\mathbf{r}} \mathbf{1}_{[0, \tau(\mathbf{r}, \overline{\mathbf{r}})]}(s) \mathbf{J}(\mathbf{r}, \overline{\mathbf{r}}) \phi(s-\tau(\mathbf{r}, \overline{\mathbf{r}}), \overline{\mathbf{r}})\right\|_{\mathbb{R}^{p}} \\
\leq \int_{\Omega} d \overline{\mathbf{r}} \mathbf{1}_{[0, \tau(\mathbf{r}, \overline{\mathbf{r}})]}(s)\|\mathbf{J}(\mathbf{r}, \overline{\mathbf{r}}) \phi(s-\tau(\mathbf{r}, \overline{\mathbf{r}}), \overline{\mathbf{r}})\|_{\mathbb{R}^{p}} \\
\leq \int_{\Omega} d \overline{\mathbf{r}} \mathbf{1}_{[0, \tau(\mathbf{r}, \overline{\mathbf{r}})]}(s)\|\| \mathbf{J}(\mathbf{r}, \overline{\mathbf{r}})\|\|_{\mathbb{R}^{p}}\|\phi(s-\tau(\mathbf{r}, \overline{\mathbf{r}}), \overline{\mathbf{r}})\|_{\mathbb{R}^{p}} \\
\leq \sqrt{p}\|\mathbf{J}\|_{L^{\infty}\left(\Omega^{2}, \mathbb{R}^{p}\right)} \int_{\Omega} d \overline{\mathbf{r}} \mathbf{1}_{[0, \tau(\mathbf{r}, \overline{\mathbf{r}})]}(s)\|\phi(s-\tau(\mathbf{r}, \overline{\mathbf{r}}), \overline{\mathbf{r}})\|_{\mathbb{R}^{p}} \\
\stackrel{\text { Holder }}{\leq} \sqrt{p}\|\mathbf{J}\|_{L^{\infty}\left(\Omega^{2}, \mathbb{R}^{p}\right)}\left(\int_{\Omega} d \overline{\mathbf{r}}\right)^{1 / \bar{q}}\left(\int_{\Omega} d \overline{\mathbf{r}} \mathbf{1}_{[0, \tau(\mathbf{r}, \overline{\mathbf{r}})]}(s)\|\phi(s-\tau(\mathbf{r}, \overline{\mathbf{r}}), \overline{\mathbf{r}})\|_{\mathbb{R}^{p}}^{q}\right)^{1 / q} .
\end{gathered}
$$

This gives:

$$
h(s) \leq \quad \leq \quad\left(\sqrt{p}\|\mathbf{J}\|_{L^{\infty}\left(\Omega^{2}, \mathbb{R}^{p}\right)}|\Omega|^{1 / \bar{q}}\right)^{q} \int_{\Omega} d \mathbf{r} \int_{\Omega} d \overline{\mathbf{r}} \quad \mathbf{1}_{[0, \tau(\mathbf{r}, \overline{\mathbf{r}})]}(s)\|\phi(s-\tau(\mathbf{r}, \overline{\mathbf{r}}), \overline{\mathbf{r}})\|_{\mathbb{R}^{p}}^{q}
$$

\footnotetext{
${ }^{4}$ Using the Cauchy-Schwarz inequality followed by the Hölder inequality.
} 
Again, we apply the Hölder inequality with $\bar{q}$ such that $q^{-1}+\bar{q}^{-1}=1$ :

$$
\begin{gathered}
\int_{0}^{t} h^{1 / q} \leq\left[\int_{0}^{t} 1\right]^{1 / \bar{q}}\left[\int_{0}^{t} h\right]^{1 / q} \leq \\
\sqrt{p}\|\mathbf{J}\|_{L^{\infty}\left(\Omega^{2}, \mathbb{R}^{p}\right)}|\Omega|^{1 / \bar{q}} t^{1 / \bar{q}}\left[\int_{0}^{t} d s \int_{\Omega} d \mathbf{r} \int_{\Omega} d \overline{\mathbf{r}} \mathbf{1}_{[0, \tau(\mathbf{r}, \overline{\mathbf{r}})]}(s)\|\phi(s-\tau(\mathbf{r}, \overline{\mathbf{r}}), \overline{\mathbf{r}})\|_{\mathbb{R}^{p}}^{q}\right]^{1 / q} \\
\stackrel{F u b i n i}{=} \sqrt{p}\|\mathbf{J}\|_{L^{\infty}\left(\Omega^{2}, \mathbb{R}^{p}\right)}|\Omega|^{1 / \bar{q}} t^{1 / \bar{q}}\left[\int_{\Omega} d \mathbf{r} \int_{\Omega} d \overline{\mathbf{r}} \int_{0}^{t} d s \mathbf{1}_{[0, \tau(\mathbf{r}, \overline{\mathbf{r}})]}(s)\|\phi(s-\tau(\mathbf{r}, \overline{\mathbf{r}}), \overline{\mathbf{r}})\|_{\mathbb{R}^{p}}^{q}\right]^{1 / q} \\
\leq \sqrt{p}\|\mathbf{J}\|_{L^{\infty}\left(\Omega^{2}, \mathbb{R}^{p}\right)}|\Omega|^{1 / \bar{q}} t^{1 / \bar{q}}\left[\int_{\Omega} d \mathbf{r} \int_{\Omega} d \overline{\mathbf{r}} \int_{-\tau_{m}}^{0} d \theta\|\phi(\theta, \overline{\mathbf{r}})\|_{\mathbb{R}^{p}}^{q}\right]^{1 / q} \\
\stackrel{\text { Fubini }}{=} \sqrt{p}\|\mathbf{J}\|_{L^{\infty}\left(\Omega^{2}, \mathbb{R}^{p}\right)}|\Omega|^{1 / \bar{q}} t^{1 / \bar{q}}\left[|\Omega| \int_{-\tau_{m}}^{0} d \theta\|\phi(\theta)\|_{\mathrm{L}^{q}}^{q}\right]^{1 / q} \\
=\sqrt{p}\|\mathbf{J}\|_{L^{\infty}\left(\Omega^{2}, \mathbb{R}^{p}\right)}|\Omega| t^{1 / \bar{q}}\|\phi\|_{\mathrm{L}^{\mathrm{q}}\left(-\tau_{\mathrm{m}}, 0 ; \mathrm{L}^{q}\right)}
\end{gathered}
$$

Finally we find:

$$
\int_{0}^{t}\left\|\mathbf{L}_{1}\left(S_{s} x+T_{0}(s) \phi\right)\right\|_{L^{q}} d s \leq \sqrt{p}\|\mathbf{J}\|_{L^{\infty}\left(\Omega^{2}, \mathbb{R}^{p}\right)}|\Omega| \max \left(t, t^{1 / \bar{q}}\right)\left\|\left[\begin{array}{l}
x \\
\phi
\end{array}\right]\right\|_{\mathcal{X}^{(q)}}
$$

which concludes the proof.

\section{The Cauchy problem}

\section{C.1 Regularity of $R$}

Lemma C. 1 Let us assume that $\mathbf{J} \in \mathrm{L}^{\infty}\left(\Omega^{2}, \mathbb{R}^{\mathrm{p} \times \mathrm{p}}\right)$. Then $\mathbf{L}_{1}$ is a continuous operator from $C^{0}\left(-\tau_{m}, 0 ; \mathrm{L}^{1}\left(\Omega, \mathbb{R}^{\mathrm{p}}\right)\right)$ to $\mathrm{L}^{\mathrm{q}}\left(\Omega, \mathbb{R}^{\mathrm{p}}\right)$.

Proof. We take $\phi \in C^{0}\left(-\tau_{m}, 0 ; \mathrm{L}^{1}\left(\Omega, \mathbb{R}^{\mathrm{p}}\right)\right)$ and write, for convenience, $\phi^{d}(\mathbf{r}, \overline{\mathbf{r}})=\phi(-\tau(\mathbf{r}, \overline{\mathbf{r}}), \overline{\mathbf{r}})$. If $\mathbf{U} \equiv \mathbf{L}_{1} \phi$, we have:

$$
\begin{aligned}
\left|U_{i}(\mathbf{r})\right|=\left|\sum_{j} \int_{\Omega} d \overline{\mathbf{r}} J_{i j}(\mathbf{r}, \overline{\mathbf{r}}) \phi_{j}^{d}(\mathbf{r}, \overline{\mathbf{r}})\right| \leq & \|\mathbf{J}\|_{L^{\infty}\left(\Omega^{2}, \mathbb{R}^{\mathrm{p} \times \mathrm{p}}\right)} \int_{\Omega} \sum_{j}\left|\phi_{j}^{d}(\mathbf{r}, \overline{\mathbf{r}})\right| d \overline{\mathbf{r}} \\
& \stackrel{\text { Cauchy-Schwarz }}{\leq} \sqrt{p}\|\mathbf{J}\|_{L^{\infty}\left(\Omega^{2}, \mathbb{R}^{\mathrm{p} \times \mathrm{p}}\right)} \int_{\Omega}\left\|\phi^{d}(\mathbf{r}, \overline{\mathbf{r}})\right\|_{\mathbb{R}^{p}} d \overline{\mathbf{r}}
\end{aligned}
$$

Also, we find:

$$
\begin{aligned}
\int_{\Omega}\left\|\phi^{d}(\mathbf{r}, \overline{\mathbf{r}})\right\|_{\mathbb{R}^{p}} d \overline{\mathbf{r}} \leq \sup _{\mathbf{r} \in \Omega} \int_{\Omega}\left\|\phi^{d}(\mathbf{r}, \overline{\mathbf{r}})\right\|_{\mathbb{R}^{p}} d \overline{\mathbf{r}} \leq \\
\sup _{\theta \in\left[-\tau_{m}, 0\right]} \int_{\Omega}\|\phi(\theta, \overline{\mathbf{r}})\|_{\mathbb{R}^{p}} d \overline{\mathbf{r}}=\sup _{\theta \in\left[-\tau_{m}, 0\right]}\|\phi(\theta)\|_{L^{1}\left(\Omega, \mathbb{R}^{\mathrm{p}}\right)} \equiv\|\phi\|_{C^{0}\left(-\tau_{m}, 0 ; \mathrm{L}^{1}\left(\Omega, \mathbb{R}^{\mathrm{p}}\right)\right)}
\end{aligned}
$$

which gives $\left|U_{i}(\mathbf{r})\right| \leq \sqrt{p}\|\mathbf{J}\|_{L^{\infty}\left(\Omega^{2}, \mathbb{R}^{\mathrm{p} \times \mathrm{p}}\right)}\|\phi\|_{C^{0}\left(-\tau_{m}, 0 ; \mathrm{L}^{1}\left(\Omega, \mathbb{R}^{\mathrm{p}}\right)\right)}$. It follows that

$$
\|\mathbf{U}\|_{\mathrm{L}^{\mathrm{q}}(\Omega, \mathbb{R})} \leq p \sqrt{p}|\Omega|^{1 / q}\|\mathbf{J}\|_{\mathrm{L}^{\infty}\left(\Omega^{2}, \mathbb{R}^{\mathrm{p} \times \mathrm{p}}\right)}\|\phi\|_{C^{0}\left(-\tau_{m}, 0 ; \mathrm{L}^{1}(\Omega, \mathbb{R})\right)}
$$

which concludes the proof.

$\mathrm{RR} \mathrm{n}^{\circ} 8020$ 


\section{C.2 The inhomogeneous equation}

Lemma C.2 Let $\left(\mathcal{T}_{0}(t)\right)$ be the $C^{0}$-semigroup with generator $\mathbf{A}_{0}=\left[\begin{array}{cc}-\mathbf{L}_{0} & 0 \\ 0 & \frac{d}{d \theta}\end{array}\right], D\left(\mathbf{A}_{0}\right)=D(\mathbf{A})$ and $\mathbf{B} \equiv\left[\begin{array}{cc}0 & \mathbf{L}_{1} \\ 0 & 0\end{array}\right] \in \mathcal{L}(D(\mathbf{A}), \mathcal{X})$. It is known from [5]/theorem 3.25] that $\mathbf{A}_{0}$ generates the strongly continuous semigroup $\mathcal{T}_{0}(t)=\left[\begin{array}{cc}S(t) & 0 \\ S_{t} & T_{0}(t)\end{array}\right] \in \mathcal{L}(\mathcal{X})$ where $S(t) \equiv e^{-\mathbf{L}_{0} t}, S_{t}, T_{0}(t)$ are defined in appendix B. Then we have:

$$
\begin{aligned}
\mathbf{T}(t) & =\mathcal{T}_{0}(t)+\int_{0}^{t} \mathcal{T}_{0}(t-s) \mathbf{B} \mathbf{T}(s) d s \\
& =\mathcal{T}_{0}(t)+\int_{0}^{t} \mathbf{T}(t-s) \mathbf{B} \mathcal{T}_{0}(s) d s
\end{aligned}
$$

on $D(\mathbf{A}) \oplus\left(\mathrm{L}^{2} \times\{0\}\right)$.

Proof. There are 3 main parts in the proof. First, we find the regularity of $\pi_{2} \mathbf{T}(t)\left[\begin{array}{l}x \\ 0\end{array}\right]$. Then we use the Miyadera-Voigt perturbation theorem to prove the lemma on $D(\mathbf{A})$. Finally, we extend the formula to $D(\mathbf{A}) \oplus\left(\mathrm{L}^{2} \times\{0\}\right)$ by continuity.

1. Let us write $\left[\begin{array}{l}f(t) \\ \xi(t)\end{array}\right] \equiv \mathbf{T}(t)\left[\begin{array}{l}x \\ 0\end{array}\right]$. We consider a sequence $D(\mathbf{A}) \ni\left[\begin{array}{c}x \\ \phi_{n}\end{array}\right] \rightarrow\left[\begin{array}{l}x \\ 0\end{array}\right]$ in $\mathcal{X}$. Then, $\left[\begin{array}{l}f_{n}(t) \\ \xi_{n}(t)\end{array}\right] \equiv \mathbf{T}(t)\left[\begin{array}{c}x \\ \phi_{n}\end{array}\right] \in D(\mathbf{A})$ is a classical solution of:

$$
\left\{\begin{array}{l}
\dot{f}_{n}=-\mathbf{L}_{0} f_{n}+\mathbf{L}_{1} \xi_{n} \\
\dot{\xi}_{n}=\frac{\partial \xi_{n}}{\partial \theta}
\end{array}\right.
$$

where the second equation is solved by $\xi_{n}(t, \theta)=H_{n}(t+\theta)$. From the initial condition $\xi_{n}(0, \theta)=\phi_{n}(\theta)$ and the belonging to $D(\mathbf{A}), \xi_{n}(t, 0)=f_{n}(t)$, we find:

$$
H_{n}(t)=\left\{\begin{array}{l}
f_{n}(t) \text { if } t \geq 0 \\
\phi_{n}(t) \text { if } t \leq 0
\end{array}\right.
$$

As $\mathbf{T}(t)\left[\begin{array}{c}x \\ \phi_{n}\end{array}\right] \rightarrow \mathbf{T}(t)\left[\begin{array}{l}x \\ 0\end{array}\right]$ in $\mathcal{X}$ for all $t \geq 0$, we have:

$$
\xi(t, \theta)=\left\{\begin{array}{lcl}
f(t+\theta) & \text { if } & t+\theta \geq 0 \\
0 & \text { otherwise }
\end{array}\right.
$$

As $\mathbf{T}$ is strongly continuous on $\mathcal{X}$, it gives $f \in C^{0}\left(\mathbb{R}_{+}, \mathrm{L}^{\mathrm{q}}\right)$ and $\xi(t, \cdot)$ has one discontinuity point if and only if $t<\tau_{m}$.

2. We apply the perturbation theorem A.5 to $\mathbf{A}_{0}+\mathbf{B}$ (see [5][theorem 3.26] and lemma B.2). We find $\forall u \in D(\mathbf{A})$ :

$$
\begin{aligned}
\mathbf{T}(t) u & =\mathcal{T}_{0}(t) u+\int_{0}^{t} \mathcal{T}_{0}(t-s) \mathbf{B} \mathbf{T}(s) u d s \\
& =\mathcal{T}_{0}(t) u+\int_{0}^{t} \mathbf{T}(t-s) \mathbf{B} \mathcal{T}_{0}(s) u d s
\end{aligned}
$$


3. Then, we extend by continuity the above formulas. Let us notice that $L^{2} \times\{0\}$ is in the closure of $D(\mathbf{A})$ in $\mathcal{X}$ and write (for example) $\delta(t)=\mathbf{T}(t)-\mathcal{T}_{0}(t)-\int_{0}^{t} \mathcal{T}_{0}(t-s) \mathbf{B T}(s) d s$. We wish to extend $\delta(t)$ to $\mathrm{L}^{2} \times\{0\}$. Let us first notice that $\delta(t)$ is bounded on $\mathrm{L}^{2} \times\{0\}$ for $\|\cdot\|_{\mathcal{X}}$. Indeed, this comes from $\mathbf{L}_{1}$ being bounded on history segment like $\pi_{2} \mathbf{T}(t)\left[\begin{array}{l}x \\ 0\end{array}\right]$. Then, we consider a sequence $D(\mathbf{A}) \ni u_{n} \rightarrow u \in \mathrm{L}^{2} \times\{0\}$ in $\mathcal{X}$. We have $\delta(t) u_{n}=0$ and as $\delta(t)$ is bounded on $D(\mathbf{A}) \oplus \mathrm{L}^{2} \times\{0\}$, we have:

$$
\|\underbrace{\delta(t) u_{n}}_{=0}-\delta(t) u\|_{\mathcal{X}} \leq K\left\|u_{n}-u\right\|_{\mathcal{X}}
$$

which gives $\delta(t) u=0$. the other formula is similar. This concludes the proof of the lemma.

We need the following estimations for the main proposition that follows.

Lemma C.3 For $f \in C_{\eta}^{0}\left(\mathbb{R}, \mathrm{L}^{\mathrm{q}}\right)$, we have:

1. $\int_{t}^{t+\epsilon} S_{t+\epsilon-r} f(r) d r \stackrel{\epsilon \rightarrow 0^{+}}{=} O\left(\epsilon^{1+1 / q}\right)$ in $\mathrm{L}^{\mathrm{q}}\left(-\tau_{\mathrm{m}}, 0 ; \mathrm{L}^{\mathrm{q}}\right)$,

2. $\forall \lambda \in \mathbb{C}$ such that $\Re \lambda \geq 0, \int_{t}^{t+\epsilon} T_{0}(t+\epsilon-r) f(r) e^{\lambda \cdot} d r \stackrel{\epsilon \rightarrow 0^{+}}{\sim} \epsilon f(t) e^{\lambda \cdot}$ in $\mathrm{L}^{\mathrm{q}}\left(-\tau_{\mathrm{m}}, 0 ; \mathrm{L}^{\mathrm{q}}\right)$.

Proof.

1. Let us write $U_{1}(t ; \cdot)=\int_{t}^{t+\epsilon} S_{t+\epsilon-r} f(r) d r$. From the definition of $S_{t}$, we find:

$$
\begin{aligned}
\left\|U_{1}(t ; \cdot)\right\|_{L^{q}\left(-\tau_{m}, 0 ; \mathrm{L}^{\mathrm{q}}\right)}^{q} & =\int_{-\tau_{m}}^{0} d \theta\left\|\int_{t}^{t+\epsilon} S(t+\epsilon-r+\theta) f(r) H(t+\epsilon-r+\theta) d r\right\|_{\mathrm{L}^{\mathrm{q}}}^{q} \\
& =\int_{-\epsilon}^{0} d \theta\left\|\int_{0}^{\theta+\epsilon} S(\epsilon-r+\theta) f(t+r) d r\right\|_{\mathrm{L}^{\mathrm{q}}}^{q} \\
& \leq \int_{-\epsilon}^{0} d \theta\left(\int_{0}^{\theta+\epsilon}\|S(\epsilon-r+\theta) f(t+r)\|_{\mathrm{L}^{\mathrm{q}}} d r\right)^{q} \\
& =O\left(\int_{-\epsilon}^{0} d \theta\left(\int_{0}^{\theta+\epsilon} 1 d r\right)^{q}\right)=O\left(\epsilon^{q+1}\right)
\end{aligned}
$$

where $H$ is the Heaviside function. Hence, we have:

$$
U_{1}(t ; \cdot)=O\left(\epsilon^{1+1 / q}\right)=o(\epsilon) .
$$

2. Let us write $U_{2}(t ; \theta)=\int_{t}^{t+\epsilon}\left(T_{0}(t+\epsilon-r)\left(f(r) e^{\lambda \cdot}\right)\right)(\theta) d r$. From the definition of $T_{0}(t)$ in appendix B,

$$
\begin{aligned}
U_{2}(t ; \theta) & =\int_{t}^{t+\epsilon} f(r) e^{\lambda(t+\theta+\epsilon-r)} H(-(t+\theta+\epsilon-r)) d r=\int_{\max (t, t+\epsilon+\theta)}^{t+\epsilon} f(r) e^{\lambda(t+\theta+\epsilon-r)} d r \\
& \equiv U_{2,1}(t ; \theta)+U_{2,2}(t ; \theta)
\end{aligned}
$$

$\mathrm{RR} \mathrm{n}^{\circ} 8020$ 
with:

$$
U_{2,1}(t ; \theta) \equiv\left\{\begin{array}{cll}
\int_{t+\epsilon+\theta}^{t+\epsilon} f(r) e^{\lambda(t+\theta+\epsilon-r)} d r & \text { if } & \theta>-\epsilon \\
0 & \text { if } \quad \theta \leq-\epsilon
\end{array}\right.
$$

and

$$
U_{2,2}(t ; \theta) \equiv\left\{\begin{array}{clc}
\int_{t}^{t+\epsilon} f(r) e^{\lambda(t+\theta+\epsilon-r)} d r & \text { if } & \theta<-\epsilon \\
0 & \text { if } \quad \theta \geq-\epsilon
\end{array}\right.
$$

For the first term, we find:

$$
\begin{aligned}
\left\|U_{2,1}(t ; \cdot)\right\|_{L^{q}\left(-\tau_{m}, 0 ; \mathrm{L}^{q}\right)}^{q} & =\int_{-\epsilon}^{0} d \theta\left\|\int_{t+\theta+\epsilon}^{t+\epsilon} f(r) e^{\lambda(t+\theta+\epsilon-r)} d r\right\|_{\mathrm{L}^{\mathrm{q}}}^{q} \\
& =O\left(\int_{-\epsilon}^{0} d \theta\left(\int_{t+\theta+\epsilon}^{t+\epsilon} 1 d r\right)^{q}\right)=O\left(\epsilon^{q+1}\right) .
\end{aligned}
$$

Hence, we have:

$$
U_{2,1}(t ; \cdot)=o(\epsilon) .
$$

For the second term, it is easy to show that:

$$
\forall \theta \in\left[-\tau_{m}, 0\right], \frac{1}{\epsilon} U_{2,2}(t ; \theta) \stackrel{\epsilon \rightarrow 0^{+}}{\rightarrow} f(t) e^{\lambda \theta} \text { in } \mathrm{L}^{\mathrm{q}}\left(-\tau_{\mathrm{m}}, 0 ; \mathrm{L}^{\mathrm{q}}\right)
$$

which concludes the proof.

Proposition C.4 For $F=\left[\begin{array}{l}f \\ 0\end{array}\right] \in C_{\eta}^{0}(\mathbb{R}, \mathcal{Y})$, we consider

$$
u(t)=\int_{-\infty}^{t} \mathbf{T}(t-r) P_{s}\left[\begin{array}{c}
f(r) \\
0
\end{array}\right] d r .
$$

Then, $u$ satisfies the following properties:

1. $u \in C_{\eta}^{0}(\mathbb{R}, \mathcal{Z})$ and $\|u\|_{C_{\eta}^{0}(\mathbb{R}, \mathcal{Z})} \leq K(\eta)\|f\|_{C_{\eta}^{0}(\mathbb{R}, \mathcal{Y})}$ with $\eta \rightarrow K(\eta)$ positive continuous,

2. $u \in C^{1}(\mathbb{R}, \mathcal{X})$,

3. $u$ satisfies $\dot{u}=\mathbf{A} u+P_{s}\left[\begin{array}{l}f \\ 0\end{array}\right]$ on $\mathcal{X}$, i.e. is a classical solution.

Proof. Choose $\gamma^{\prime}>\gamma$ such that the spectral splitting (see section 3.4) is still valid with $\gamma^{\prime}$. It gives

$$
\left\|\mathbf{T}(t) P_{s}\right\|_{\mathcal{X}} \leq M e^{-\gamma^{\prime} t}, t>0 .
$$

Note that the group $S(t)=e^{-\mathbf{L}_{0} t}$ satisfies:

$$
\|S(t)\|_{\mathcal{L}\left(\mathrm{L}^{\mathrm{q}}\right)} \leq e^{-l t}, l>\gamma^{\prime}, \forall t .
$$

Let us prove that $u(t)$ exists for all $t \in \mathbb{R}$. From (44), we find $\left\|\mathbf{T}(t-r) P_{s}\left[\begin{array}{c}f(r) \\ 0\end{array}\right]\right\|_{\mathcal{X}} \leq$ $M e^{-\gamma^{\prime}(t-r)+\eta|r|}\|f\|_{C_{\eta}^{0}(\mathbb{R}, \mathcal{Y})}$ which gives:

$$
\|u(t)\|_{\mathcal{X}} \leq M\|f\|_{C_{\eta}^{0}(\mathbb{R}, \mathcal{Y})} \int_{-\infty}^{t} e^{-\gamma^{\prime}(t-r)+\eta|r|} d r .
$$


We change of variable in the integral to find

$$
\int_{-\infty}^{t} e^{-\gamma^{\prime}(t-r)+\eta|r|} d r=\int_{-\infty}^{0} e^{\gamma^{\prime} r+\eta|t+r|} d r \leq \int_{-\infty}^{0} e^{\gamma^{\prime} r+\eta|t|+\eta|r|} d r=\frac{e^{\eta|t|}}{\gamma^{\prime}-\eta}
$$

This gives:

$$
\|u(t)\|_{\mathcal{X}} \leq M\|f\|_{C_{\eta}^{0}(\mathbb{R}, \mathcal{Y})} \frac{e^{\eta|t|}}{\gamma^{\prime}-\eta}
$$

1. From lemma C.2, we find

$$
\begin{aligned}
u(t) & =\int_{-\infty}^{t}\left(\mathcal{T}_{0}(t-r) P_{s}\left[\begin{array}{c}
f(r) \\
0
\end{array}\right]+\int_{0}^{t-r} \mathcal{T}_{0}(t-r-v) \mathbf{B T}(v) P_{s}\left[\begin{array}{c}
f(r) \\
0
\end{array}\right] d v\right) d r \\
& \equiv u_{1}(t)+u_{2}(t) .
\end{aligned}
$$

We shall prove Point 1 . for each $u_{i}(t), i=1,2$.

Case of $u_{1}$. The first term is:

$$
u_{1}(t)=\int_{-\infty}^{t} \mathcal{T}_{0}(t-r) P_{s}\left[\begin{array}{c}
f(r) \\
0
\end{array}\right] d r
$$

Recall from proposition 3.8 that

$$
P_{s}(u)=u-\sum_{i}\left\langle\left\langle\psi_{i}, u\right\rangle\right\rangle \phi_{i}
$$

where $\phi_{i}=\left[\begin{array}{c}x_{i} \\ x_{i} e^{\lambda_{i} \theta}\end{array}\right]$ are the generalized eigenvectors of $\mathbf{A}$ for the eigenvalues $\lambda_{i}$ such that $\Re \lambda_{i} \geq 0$. Write $\psi_{i}=\left[\begin{array}{c}y_{i} \\ \pi_{2} \psi_{i}\end{array}\right], f_{i}(r) \equiv\left\langle y_{i}, f(r)\right\rangle_{\mathrm{L}^{2}} x_{i}$ and $H$ the Heaviside function. Note that:

$$
\left\|f_{i}(r)\right\|_{\mathcal{X}}=O\left(\|f(r)\|_{\mathcal{X}}\right)=O\left(e^{\eta|r|}\right)
$$

because $\mathcal{X}^{(q)} \hookrightarrow \mathcal{X}^{(2)}$ for $q \geq 2$. Then, we have:

$$
\begin{gathered}
\mathcal{T}_{0}(t-r) P_{s}\left[\begin{array}{c}
f(r) \\
0
\end{array}\right]=\mathcal{T}_{0}(t-r)\left[\begin{array}{c}
f(r)-\sum_{i} f_{i}(r) \\
-\sum_{i} f_{i}(r) e^{\lambda_{i} \theta}
\end{array}\right] \\
S(t-r)\left(f(r)-\sum_{i} f_{i}(r)\right) \\
=\left[\begin{array}{c}
S(t-r+\theta)) \\
S_{t-r}\left(f(r)-\sum_{i} f_{i}(r)\right)-\sum_{i} f_{i}(r) e^{\lambda_{i}(t-r+\theta)} H(-(t-r)
\end{array}\right] .
\end{gathered}
$$

We shall see that the second component is continuous in $\theta$. It is then easy to see that the two components are equal when $\theta=0$. We have for the second component:

$$
\theta \rightarrow \int_{-\infty}^{t+\theta} S(t-r+\theta)\left(f(r)-\sum_{i} f_{i}(r)\right) d r-\sum_{i} \int_{t+\theta}^{t} f_{i}(r) e^{\lambda_{i}(t-r+\theta)} d r
$$

$\mathrm{RR} \mathrm{n}^{\circ} 8020$ 
The second term is $C^{1}$ on $\left[-\tau_{m}, 0\right]$. We wish to apply the dominated convergence theorem to the first integral term. Hence, we need to bound the norm of the derivative of its integrand by an integrable function of $r$. This derivative is

$$
-\mathbf{L}_{0} S(t-r+\theta)\left(f(r)-\sum_{i} f_{i}(r)\right)
$$

which is bounded ${ }^{5}$ by $K\left\|\left|\mathbf{L}_{0}\right|\right\|_{\mathrm{L}^{2}}\|f\|_{C_{\eta}^{0}(\mathbb{R}, \mathcal{Y})} e^{l \tau_{m}} e^{-l\left(t-r+\tau_{m}\right)+\eta|r|}$ which is $r$-integrable. Hence, the dominated convergence theorem shows that the second component belongs to $C^{1}\left(-\tau_{m}, 0 ; \mathrm{L}^{\mathrm{q}}\right)$ for all time $t$. This implies that

$$
\forall t \in \mathbb{R}, u_{1}(t) \in \mathcal{Z}
$$

because $\pi_{1} u_{1}(t)=\left(\pi_{2} u_{1}\right)(0)$. Finally:

$$
\begin{gathered}
\left\|u_{1}(t)\right\|_{\mathcal{Z}} \leq\left\|\int_{-\infty}^{t} S(t-r)\left(f(r)-\sum_{i} f_{i}(r)\right) d r\right\|_{\mathrm{L}^{\mathrm{q}}} \\
+\left\|\int_{-\infty}^{t} S_{t-r}\left(f(r)-\sum_{i} f_{i}(r)\right)-\sum_{i} f_{i}(r) e^{\lambda_{i}(t-r+\theta)} H(-(t-r+\theta)) d r\right\|_{\mathrm{W}^{1, \mathrm{q}}\left(-\tau_{\mathrm{m}}, 0 ; \mathrm{L}^{\mathrm{q}}\right)} \\
\leq\left\|\int_{-\infty}^{t} S(t-r)\left(f(r)-\sum_{i} f_{i}(r)\right) d r\right\|_{\mathrm{L}^{\mathrm{q}}} \\
+\left\|\int_{-\infty}^{t} S_{t-r}\left(f(r)-\sum_{i} f_{i}(r)\right)\right\|_{\mathrm{W}^{1, \mathrm{q}}\left(-\tau_{\mathrm{m}}, 0 ; \mathrm{L}^{\mathrm{q}}\right)} \\
+\left\|\int_{-\infty}^{t} \sum_{i} f_{i}(r) e^{\lambda_{i}(t-r+\theta)} H(-(t-r+\theta)) d r\right\|_{\mathrm{W}^{1, \mathrm{q}}\left(-\tau_{\mathrm{m}}, 0 ; \mathrm{L}^{\mathrm{q}}\right)}
\end{gathered}
$$

The first term is bounded by $K\|f\|_{C_{\eta}^{0}(\mathbb{R}, \mathcal{Y})} \int_{-\infty}^{t} e^{-l(t-r)+\eta|r|} d r \leq K\|f\|_{C_{\eta}^{0}(\mathbb{R}, \mathcal{Y})} \int_{-\infty}^{t} e^{-\gamma^{\prime}(t-r)+\eta|r|} d r \leq$ $K\|f\|_{C_{\eta}^{0}(\mathbb{R}, \mathcal{Y})} \frac{e^{\eta|t|}}{\gamma^{\prime}-\eta}$. Let us write:

$$
\left\|f(r)-\sum_{i} f_{i}(r)\right\|_{\mathcal{X}} \leq K_{f}\|f\|_{C_{\eta}^{0}(\mathbb{R}, \mathcal{Y})} e^{\eta|r|}
$$

for some constant $K_{f}$. For the second term in (49), we have:

$$
\begin{aligned}
& \| \int_{-\infty}^{t} S_{t-r}\left(f(r)-\sum_{i} f_{i}(r)\right) d r \|_{\mathrm{Lq}^{\mathrm{q}}\left(-\tau_{\mathrm{m}}, 0 ; \mathrm{Lq}\right)}^{q} \\
&= \int_{-\tau_{m}}^{0}\left\|\int_{-\infty}^{t+\theta} S(t-r+\theta)\left(f(r)-\sum_{i} f_{i}(r)\right) d r\right\|_{\mathrm{L}^{\mathrm{q}}}^{q} d \theta \\
& \leq \int_{-\tau_{m}}^{0}\left(\int_{-\infty}^{t+\theta} K_{f}\|f\|_{C_{\eta}^{0}(\mathbb{R}, \mathcal{Y})} e^{\eta|t|-l(t-r+\theta)} d r\right)^{q} d \theta \\
& \leq \tau_{m}\left(K_{f}\|f\|_{C_{\eta}^{0}(\mathbb{R}, \mathcal{Y})} \frac{e^{\eta|t|+l \tau_{m}}}{\gamma^{\prime}-\eta}\right)^{q}
\end{aligned}
$$

\footnotetext{
${ }^{5}$ for some constant $K$ depending on $x_{i}, y_{i}$.
} 
and

$$
\begin{aligned}
& \left\|\frac{d}{d \theta} \int_{-\infty}^{t} S_{t-r}\left(f(r)-\sum_{i} f_{i}(r)\right) d r\right\|_{\mathrm{L}^{\mathrm{q}}\left(-\tau_{\mathrm{m}}, 0 ; \mathrm{L}^{\mathrm{q}}\right)}^{q} \\
& =\int_{-\tau_{m}}^{0}\left\|\left(f(t+\theta)-\sum_{i} f_{i}(t+\theta)\right)-\int_{-\infty}^{t+\theta} \mathbf{L}_{0} S(t-r+\theta)\left(f(r)-\sum_{i} f_{i}(r)\right) d r\right\|_{L^{q}}^{q} d \theta \\
& \stackrel{|a+b|^{q} \leq 2^{q-1}\left(|a|^{q}+|b|^{q}\right)}{\leq} 2^{q-1} \int_{-\tau_{m}}^{0}\left\|f(t+\theta)-\sum_{i} f_{i}(t+\theta)\right\|_{\mathrm{L}^{q}}^{q} \\
& +2^{q-1}\left(\int_{-\infty}^{t+\theta}\left\|\mathbf{L}_{0} S(t-r+\theta)\left(f(r)-\sum_{i} f_{i}(r)\right)\right\|_{\mathrm{L}^{q}} d r\right)^{q} d \theta \\
& \leq K_{f}^{q}\|f\|_{C_{\eta}^{0}(\mathbb{R}, \mathcal{Y})}^{q}\left(\int_{-\tau_{m}}^{0} e^{q \eta|t+\theta|} d \theta+\left\|\left|\mathbf{L}_{0} \|^{q}\right|^{q} \int_{-\tau_{m}}^{0} d \theta\left(\int_{-\infty}^{t+\theta} e^{-l(t-r+\theta)+\eta|r|} d r\right)^{q}\right)\right. \\
& \leq K_{f}^{q}\|f\|_{C_{\eta}^{0}(\mathbb{R}, \mathcal{Y})}^{q} e^{q \eta|t|} \tau_{m} e^{q \eta \tau_{m}}\left(1+\left(\frac{\|\left|\mathbf{L}_{0}\right|||}{\gamma^{\prime}-\eta}\right)^{q}\right) \text {. }
\end{aligned}
$$

The third term in (50) is very similar. This allows to conclude that:

$$
\left\|u_{1}(t)\right\|_{\mathcal{Z}} \leq K_{1}(\eta)\|f\|_{C_{\eta}^{0}(\mathbb{R}, \mathcal{Y})} e^{\eta|t|}
$$

Finally, let us write for $t>s$ :

$$
\begin{aligned}
u_{1}(t)-u_{1}(s)=\int_{-\infty}^{t}\left(\mathcal{T}_{0}(t-r)-\mathcal{T}_{0}(s-r)\right) P_{s}\left[\begin{array}{r}
f(r) \\
0
\end{array}\right] d r & \\
& +\int_{s}^{t} \mathcal{T}_{0}(t-r) P_{s}\left[\begin{array}{c}
f(r) \\
0
\end{array}\right] d r
\end{aligned}
$$

Using the same arguments as above, it is straightforward to show that:

$$
\left\|\int_{s}^{t} \mathcal{T}_{0}(t-r)\left[\begin{array}{c}
f(r) \\
0
\end{array}\right] d r\right\|_{\mathcal{Z}}=O(|t-s|)
$$

and

$$
\left\|\int_{-\infty}^{t}\left(\mathcal{T}_{0}(t-r)-\mathcal{T}_{0}(s-r)\right)\left[\begin{array}{c}
f(r) \\
0
\end{array}\right] d r\right\|_{\mathcal{Z}}=O(|t-s|)
$$

because $t \rightarrow S(t)$ is $C^{1}$. This shows that $\left\|u_{1}(t)-u_{1}(s)\right\|_{\mathcal{Z}} \leq K|t-s|$, hence $t \rightarrow u_{1}(t)$ is continuous in $\mathcal{Z}$. It follows that

$$
u_{1} \in C_{\eta}^{0}(\mathbb{R}, \mathcal{Z})
$$

Case of $u_{2}$. We start with the introduction of convenient notations:

$$
\left[\begin{array}{c}
h(v, r) \\
0
\end{array}\right] \equiv \mathbf{B T}(v) P_{s}\left[\begin{array}{c}
f(r) \\
0
\end{array}\right]
$$

Note from (44) that

$$
\|h(v, r)\|_{\mathrm{L}^{\mathrm{q}}} \leq K\|f\|_{C_{\eta}^{0}(\mathbb{R}, \mathcal{Y})} e^{-\gamma^{\prime} v+\eta|r|}
$$

$\mathrm{RR} \mathrm{n}^{\circ} 8020$ 
for some constant $K$. It follows that $u_{2}$ is given by:

$$
u_{2}(t)=\int_{-\infty}^{t} d r \int_{0}^{t-r} d v\left[\begin{array}{c}
S(t-r-v) h(v, r) \\
S_{t-r-v} h(v, r)
\end{array}\right]
$$

and

$$
\begin{aligned}
\pi_{2} u_{2}(t ; \theta) & =\int_{-\infty}^{t} d r \int_{0}^{t-r} d v S(t-r-v+\theta) h(v, r) H(t-r-v+\theta) \\
& =\int_{-\infty}^{t+\theta} d r \int_{0}^{t-r+\theta} d v S(t-r-v+\theta) h(v, r) .
\end{aligned}
$$

We want to show that this function is $C^{1}$ on $\left[-\tau_{m}, 0\right]$ by applying the dominated convergence theorem. The integrand is $C^{1}$ in $\theta$. Taking the derivative w.r.t. $\theta$ gives:

$$
h(t-r+\theta, r)-\int_{0}^{t-r+\theta} d v \mathbf{L}_{0} S(t-r-v+\theta) h(v, r) .
$$

The first term is bounded by $K\|f\|_{C_{\eta}^{0}(\mathbb{R}, \mathcal{Y})} e^{-\gamma^{\prime}(t-r)+\eta|r|}$ and the second term is bounded by

$$
K\|f\|_{C_{\eta}^{0}(\mathbb{R}, \mathcal{Y})}||\left|\mathbf{L}_{0}\right||| \int_{0}^{t-r+\theta} d v e^{-l\left(t-r-v-\tau_{m}\right)} e^{-\gamma^{\prime} v+\eta|r|}=O\left(e^{-\gamma^{\prime}(t-r)+\eta|r|}\right) .
$$

Hence, the derivative is $O\left(e^{-\gamma^{\prime}(t-r)+\eta|r|}\right)$ which is $r$-integrable. It follows from the dominated convergence theorem that $\pi_{2} u_{2}(t)$ is $C^{1}$ on $\left[-\tau_{m}, 0\right]$ for all time $t$. We find that:

$$
\forall t \in \mathbb{R}, u_{2}(t) \in \mathcal{Z} .
$$

We can now compute the norm $\left\|u_{2}(t)\right\|_{\mathcal{Z}}$ :

$$
\begin{aligned}
\left\|u_{2}(t)\right\|_{\mathcal{Z}}=\| \int_{-\infty}^{t} d r \int_{0}^{t-r} S( & -r-v) h(v, r) d v \|_{\mathrm{L}^{\mathrm{q}}} \\
+ & \left\|\int_{-\infty}^{t} d r \int_{0}^{t-r} S_{t-r-v} h(v, r) d v\right\|_{\mathrm{W}^{1, \mathrm{q}}\left(-\tau_{\mathrm{m}}, 0 ; \mathrm{L}^{\mathrm{q}}\right)}
\end{aligned}
$$

From computations similar to the ones for $\left\|u_{1}(t)\right\|_{\mathcal{Z}}$ and by using the bound (51), we find that

$$
\left\|u_{2}(t)\right\|_{\mathcal{Z}} \leq M(\eta)\|f\|_{C_{\eta}^{0}(\mathbb{R}, \mathcal{Y})} e^{\eta|t|}
$$

where $\eta \rightarrow M(\eta)$ is a positive continuous function of $\eta$. Finally, we have for $\delta>0$ (for example):

$$
\begin{gathered}
u_{2}(t+\delta)-u_{2}(t)= \\
\int_{-\infty}^{t} d r\left(\int_{0}^{t-r} d v\left[\mathcal{T}_{0}(t+\delta-r-v)-\mathcal{T}_{0}(t-r-v)\right]\left[\begin{array}{c}
h(v, r) \\
0
\end{array}\right]\right. \\
\left.+\int_{t-r}^{t+\delta-r} d v \mathcal{T}_{0}(t+\delta-r-v)\left[\begin{array}{c}
h(v, r) \\
0
\end{array}\right]\right)+\int_{t}^{t+\delta} d r \int_{0}^{t+\delta-r} d v \mathcal{T}_{0}(t+\delta-r-v)\left[\begin{array}{c}
h(v, r) \\
0
\end{array}\right] .
\end{gathered}
$$

By bounding each term, it can be shown, albeit being lengthy, that $\left\|u_{2}(t+\delta)-u_{2}(t)\right\| \stackrel{\delta \sim 0}{=}$ $O(|\delta|)$. It follows that 


$$
u_{2} \in C^{0}(\mathbb{R}, \mathcal{Z})
$$

Combining the results for $u_{1}$ and $u_{2}$, we have shown that $u \in C_{\eta}^{0}(\mathbb{R}, \mathcal{Z})$ and that

$$
\|u\|_{C_{\eta}^{0}(\mathbb{R}, \mathcal{Z})} \leq K(\eta)\|f\|_{C_{\eta}^{0}(\mathbb{R}, \mathcal{Y})}
$$

for some positive continuous function $\eta \rightarrow K(\eta)$.

2. As in Point 1., we have:

$$
\begin{aligned}
u(t) & =\int_{-\infty}^{t}\left[\begin{array}{c}
S(t-r)\left(f(r)-\sum_{i} f_{i}(r)\right) \\
S_{t-r}\left(f(r)-\sum_{i} f_{i}(r)\right)-\sum_{i} f_{i}(r) e^{\lambda_{i}(t-r+\theta)} H(-(t-r+\theta))
\end{array}\right] d r \\
& +\int_{-\infty}^{t} d r \int_{0}^{t-r} d v\left[\begin{array}{c}
S(t-r-v) h(v, r) \\
S_{t-r-v} h(v, r)
\end{array}\right] .
\end{aligned}
$$

The only difficulty in showing that $t \rightarrow u(t)$ is $C^{1}$ in $\mathcal{X}$ comes from the second component because $t \rightarrow S(t)$ is analytical on $\mathrm{L}^{\mathrm{q}}$. To keep the proof small, we shall only prove that

$$
t \rightarrow U(t) \equiv \int_{-\infty}^{t} S_{t-r} f(r) d r
$$

is $C^{1}$, the other terms being very similar. We write:

$$
U(t ; \theta)=\int_{-\infty}^{t+\theta} S(t+\theta-r) f(r) d r .
$$

Using the dominated convergence theorem, we find:

$$
\frac{d}{d t} U(t ; \theta)=f(t+\theta)-\int_{-\infty}^{t+\theta} \mathbf{L}_{0} S(t+\theta-r) f(r) d r=f(t+\theta)-\mathbf{L}_{0} U(t ; \theta) .
$$

As $\theta \rightarrow \frac{d}{d t} U(t ; \theta) \in \mathrm{L}^{\mathrm{q}}\left(-\tau_{\mathrm{m}}, 0 ; \mathrm{L}^{\mathrm{q}}\right)$, we have that

$$
U^{\prime}(t)=-\mathbf{L}_{0} U(t)+f(t+\cdot) .
$$

Doing similar estimations with the other terms of the second component of $u(t)$, we find that:

$$
u \in C^{1}(\mathbb{R}, \mathcal{X}) .
$$

3. Let us consider $a>0$. From the definition of $u(t)$, we find:

$$
u(t+a)=\mathbf{T}(a) u(t)+\int_{t}^{t+a} \mathbf{T}(a+t-r) P_{s}\left[\begin{array}{c}
f(r) \\
0
\end{array}\right] d r .
$$

As $u$ is in $C^{1}(\mathbb{R}, \mathcal{X}) \cap C^{0}(\mathbb{R}, \mathcal{Z})$, we can take the derivative w.r.t. $a$ and find:

$$
\frac{d}{d a} u(t+a)=\frac{d}{d t} u(t+a)=\mathbf{A} u(t+a)+\frac{d}{d a} \int_{t}^{t+a} \mathbf{T}(a+t-r) P_{s}\left[\begin{array}{c}
f(r) \\
0
\end{array}\right] d r .
$$

$\mathrm{RR} \mathrm{n}^{\circ} 8020$ 
Then, taking the limit $a \rightarrow 0$ in $\mathcal{X}$ :

$$
\dot{u}(t)=\mathbf{A} u(t)+\lim _{a \rightarrow 0} \frac{d}{d a} \int_{t}^{t+a} \mathbf{T}(a+t-r) P_{s}\left[\begin{array}{c}
f(r) \\
0
\end{array}\right] d r .
$$

Hence we need to compute

$$
\lim _{a \rightarrow 0^{+}} \frac{1}{a} \int_{t}^{t+a} \mathbf{T}(a+t-r) P_{s}\left[\begin{array}{c}
f(r) \\
0
\end{array}\right] d r .
$$

Let us write $u_{a}(t)=\int_{t}^{t+a} \mathbf{T}(a+t-r) P_{s}\left[\begin{array}{c}f(r) \\ 0\end{array}\right] d r$. As in Point 2., we find:

$$
\begin{array}{r}
u_{a}(t)=\int_{t}^{t+a}\left[\begin{array}{r}
S(t+a-r) \\
\left.S_{t+a-r}\left(f(r)-\sum_{i} f_{i}(r)\right)-\sum_{i} f_{i}(r)\right) \\
\left.-\sum_{i} f_{i}(r) T_{0}(t+a-r) e^{\lambda_{i}} \cdot\right]
\end{array}\right] d r \\
+\int_{t}^{t+a} d r \int_{0}^{t+a-r} d v\left[\begin{array}{c}
S(t+a-r-v) h(v, r) \\
S_{t+a-r-v} h(v, r)
\end{array}\right]
\end{array}
$$

First term. We start by the first component which gives the limit (it is differentiable):

$$
\lim _{a \rightarrow 0^{+}} \frac{1}{a} \int_{t}^{t+a} S(t+a-r)\left(f(r)-\sum_{i} f_{i}(r)\right)=f(t)-\sum_{i} f_{i}(t) .
$$

Using lemma C.3, we find

$$
\begin{aligned}
\lim _{a \rightarrow 0^{+}} \frac{1}{a} \int_{t}^{t+a} S_{t+a-r}\left(f(r)-\sum_{i} f_{i}(r)\right)-\sum_{i} f_{i}(r) T_{0}(t+a-r) e^{\lambda_{i}} . & \\
& =-\sum_{i} f_{i}(r) T_{0}(t+a-r) e^{\lambda_{i}} .
\end{aligned}
$$

Hence, the limit of the first term is $P_{s}\left[\begin{array}{c}f(t) \\ 0\end{array}\right]$.

Second term. Using lemma C.3, we find that the second term is $o\left(a^{2}\right)$.

Because $u \in C^{1}(\mathbb{R}, \mathcal{X})$, we have shown that $\forall t \in \mathbb{R}$,

$$
\dot{u}=\mathbf{A} u+P_{s}\left[\begin{array}{l}
f \\
0
\end{array}\right] .
$$

This concludes the proof of the proposition.

\section{C.3 The inhomogeneous equation (Parabolic case)}

We would like to show that the center manifold is attracting when the unstable spectrum is empty $\Sigma_{u}(\mathbf{A})=\emptyset$. This requires to check two properties like we did in appendix C.2. We start with a definition for a given Banach space $\mathcal{E}$ :

$$
\mathcal{F}_{\eta}(\mathbb{R}, \mathcal{E}) \equiv\left\{\phi \in C^{0}(\mathbb{R}, \mathcal{E}),\|\phi\|_{\mathcal{F}_{\eta}(\mathbb{R}, \mathcal{E})} \equiv \sup _{t \in \mathbb{R}} e^{\eta t}\|\phi(t)\|_{\mathcal{E}}<\infty\right\} .
$$

Then, we have the following proposition (analogue to proposition 4.3) 
Proposition C.5 Let us assume that the unstable spectrum is empty $\Sigma_{u}(\mathbf{A})=\emptyset$. Define for all integer $2 \leq q<\infty, \mathcal{Y}_{h}=P_{h} \mathcal{Y}^{(q)}, \mathcal{Z}_{h}=P_{h} \mathcal{Z}^{(q)}$. For any $\eta \in[0, \gamma]$,

1. and for any $u_{0} \in \mathcal{Z}_{h}$, the problem $\dot{u}=\mathbf{A} u$ with initial condition $u_{0}$ has a unique solution $u \in C^{0}\left(\mathbb{R}^{+}, \mathcal{Z}_{h}\right)$ and $\|u(t)\|_{\mathcal{Z}} \leq c_{\eta} e^{-\eta t}$ for all $t \geq 0$ and some positive constant $c_{\eta}$.

2. and for any function $F=P_{h}\left[\begin{array}{l}f \\ 0\end{array}\right] \in \mathcal{F}_{\eta}\left(\mathbb{R}, \mathcal{Y}_{h}\right)$, the problem

$$
\dot{u}=\mathbf{A} u+F(t)
$$

has a unique solution $u=\mathbf{K}_{h} F \in \mathcal{F}_{\eta}\left(\mathbb{R}, \mathcal{Z}_{h}\right)$ and

$$
\left\|\mid \mathbf{K}_{h}\right\|_{\mathcal{L}\left(\mathcal{F}_{\eta}\left(\mathbb{R}, \mathcal{Y}_{h}\right), \mathcal{F}_{\eta}\left(\mathbb{R}, \mathcal{Z}_{h}\right)\right)} \leq C(\eta)
$$

with $C \in C^{0}([0, \gamma], \mathbb{R})$.

Proof. Choose $\gamma^{\prime}>\gamma$ such that the spectral splitting (see section 3.4) is still valid with $\gamma^{\prime}$.

1. Let us write $u_{0}=\left[\begin{array}{l}x_{0} \\ \phi_{0}\end{array}\right]$. The solution $u$ is given by $u(t)=\mathbf{T}(t) u_{0}$. From $\mathcal{Z}^{(q)}=D\left(\mathbf{A}_{(q)}\right)$, we find that $u$ is a strong solution and that $u(t) \in \mathcal{Z}_{h}$. Using the same trick as in proposition C.4, we write $u(t)=\mathcal{T}_{0}(t) u_{0}+\int_{0}^{t} \mathcal{T}_{0}(t-s) \mathbf{B T}(s) u_{0} d s \equiv u_{1}(t)+u_{2}(t)$. For $u_{0} \in \mathcal{Z}_{h}$, we find $u_{1}(t) \in \mathcal{Z}_{h}$. Also, for $t>\tau_{m}$, we have $u_{1}(t)=\left[\begin{array}{c}S(t) x_{0} \\ S_{t} x_{0}\end{array}\right]$, where $S(t) \equiv e^{-\mathbf{L}_{0} t}$, which gives $\left\|u_{1}(t)\right\|_{\mathcal{Z}}=O\left(e^{-l t}\right)$. Let us look at the expression $u_{2}(t)$. From $\mathbf{B T}(s) u_{0} \equiv\left[\begin{array}{c}h(s) \\ 0\end{array}\right]$, we have $\|h(s)\|_{\mathrm{L}^{\mathrm{q}}} \leq K e^{-\gamma^{\prime} s}\left\|u_{0}\right\|_{\mathcal{Z}}$. We find

$$
u_{2}(t)=\int_{0}^{t}\left[\begin{array}{c}
S(t-s) h(s) \\
S_{t-s} h(s)
\end{array}\right] d s
$$

which belongs to $\mathcal{Z}$. Using the estimate of $h$, it is straightforward to show that $\left\|u_{2}(t)\right\|_{\mathcal{Z}}=$ $O\left(e^{-\eta t}\right)$ (see the proof of proposition C.4). This allows to conclude the proof of the first part.

2. Let us consider

$$
u(t)=\int_{-\infty}^{t} \mathbf{T}(t-r) P_{s}\left[\begin{array}{c}
f(r) \\
0
\end{array}\right] d r .
$$

As $\mathcal{F}_{\eta}\left(\mathbb{R}, \mathcal{Y}_{h}\right)$ is continuously embedded in $C_{\eta}^{0}\left(\mathbb{R}, \mathcal{Y}_{h}\right)$, proposition 4.3 shows that $u \in$ $C_{\eta}^{0}\left(\mathbb{R}, \mathcal{Z}_{h}\right) \cap C^{1}(\mathbb{R}, \mathcal{X})$ and that $u$ is a classical solution of (54). In order to prove that

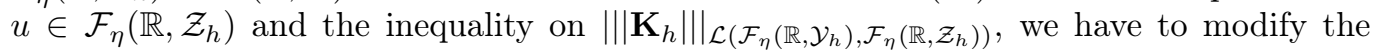
estimates in proposition 4.3 although the general plan of the proof is exactly the same. Changing the norms $\|\cdot\|_{C_{\eta}^{0}(\mathbb{R}, \cdot)}$ into $\|\cdot\|_{\mathcal{F}_{\eta}(\mathbb{R}, \cdot)}$ in the estimates of proposition 4.3 is straightforward but lengthy and we shall only show how to do this for the first one of them, the other being very similar. The inequality we shall consider is the first that arises in the proof of proposition 4.3. We find: $\left\|\mathbf{T}(t-r) P_{s}\left[\begin{array}{c}f(r) \\ 0\end{array}\right]\right\|_{\mathcal{X}} \leq M e^{-\gamma^{\prime}(t-r)-\eta r}\|f\|_{\mathcal{F}_{\eta}\left(\mathbb{R}, \mathcal{Y}_{h}\right)}$ which gives:

$$
\|u(t)\|_{\mathcal{X}} \leq M\|f\|_{\mathcal{F}_{\eta}\left(\mathbb{R}, \mathcal{Y}_{h}\right)} \int_{-\infty}^{t} e^{-\gamma^{\prime}(t-r)-\eta r} d r \leq M\|f\|_{\mathcal{F}_{\eta}\left(\mathbb{R}, \mathcal{Y}_{h}\right)} \frac{e^{-\eta t}}{\gamma^{\prime}-\eta}
$$

Following the proof of proposition 4.3, we can prove of the second part of the present proposition. 


\section{Contents}

1 Introduction $\quad 3$

2 Preliminaries $\quad 5$

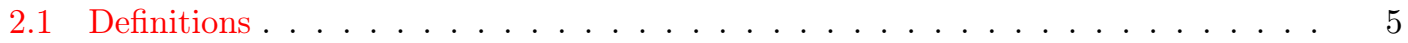

2.2 Results concerning dynamics . . . . . . . . . . . . . . . . 6

3 Linear analysis $\quad 6$

3.1 Semigroup properties from the spectral study . . . . . . . . . . . . . . 6

3.2 Generalized eigenspaces . . . . . . . . . . . . . . . . . . 10

3.3 Spectral projector on generalized eigenspaces . . . . . . . . . . . . . 11

3.4 Phase space decomposition . . . . . . . . . . . . . . . . . . . 14

4 Center manifold reduction $\quad \mathbf{1 5}$

4.1 Formulation as a Cauchy problem . . . . . . . . . . . . . . . . . 16

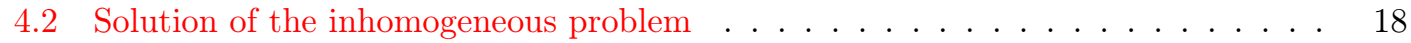

4.3 Center manifold and reduced equation . . . . . . . . . . . . . . . . . . . . . 20

4.4 Normal form of the Pitchfork bifurcation . . . . . . . . . . . . . . . . . . 21

5 Conclusion $\quad 22$

$\begin{array}{lr}\text { A Operators and their spectra } & \mathbf{2 3}\end{array}$

$\begin{array}{lr}\text { B Regularity } & 25\end{array}$

C The Cauchy problem $\quad \mathbf{2 7}$

C.1 Regularity of $\mathbf{R} \ldots \ldots \ldots \ldots \ldots \ldots \ldots$

C.2 The inhomogeneous equation . . . . . . . . . . . . . . . . . . 28

C.3 The inhomogeneous equation (Parabolic case) . . . . . . . . . . . . 36

\section{References}

[1] O. Arino, ML Hbid, and E.A. Dads. Delay differential equations and applications. Springer, 2006.

[2] O. Arino and E. Sanchez. Linear theory of abstract functional differential equations of retarded type. Journal of mathematical analysis and applications, 191:547-571, 1995.

[3] Fatihcan M. Atay and Axel Hutt. Stability and bifurcations in neural fields with finite propagation speed and general connectivity. SIAM Journal on Applied Mathematics, 65(2):644$666,2005$.

[4] Fatihcan M. Atay and Axel Hutt. Neural fields with distributed transmission speeds and long-range feedback delays. SIAM Journal of Applied Dynamical Systems, 5(4):670-698, 2006.

[5] A. Bátkai and S. Piazzera. Semigroups for delay equations. AK Peters, Ltd., 2005.

[6] R. Ben-Yishai, RL Bar-Or, and H. Sompolinsky. Theory of orientation tuning in visual cortex. Proceedings of the National Academy of Sciences, 92(9):3844-3848, 1995. 
[7] I. Bojak and D.T.J. Liley. Axonal velocity distributions in neural field equations. PLOS Comp. Bio., 2010.

[8] P.C. Bressloff, N.W. Bressloff, and J.D. Cowan. Dynamical mechanism for sharp orientation tuning in an integrate-and-fire model of a cortical hypercolumn. Neural computation, 12(11):2473-2511, 2000.

[9] P.C. Bressloff, J.D. Cowan, M. Golubitsky, P.J. Thomas, and M.C. Wiener. Geometric visual hallucinations, euclidean symmetry and the functional architecture of striate cortex. Phil. Trans. R. Soc. Lond. B, 306(1407):299-330, March 2001.

[10] P.C. Bressloff and Z.P. Kilpatrick. Nonlocal ginzburg-landau equation for cortical pattern formation. Physical Review E, 78(4):41916:1-16, 2008.

[11] P. Clément, O. Diekmann, M. Gyllenberg, H. Heijmans, and HR Thieme. Perturbation theory for dual semigroups. Mathematische Annalen, 277(4):709-725, 1988.

[12] B.D. Coleman and V.J. Mizel. Norms and semi-groups in the theory of fading memory. Archive for Rational Mechanics and Analysis, 23(2):87-123, 1966.

[13] S. Coombes, N.A Venkov, L. Shiau, I. Bojak, D.T.J. Liley, and C.R. Laing. Modeling electrocortical activity through local approximations of integral neural field equations. Physical Review E, 76(5):51901, 2007.

[14] Stephen Coombes. Waves, bumps, and patterns in neural fields theories. Biological Cybernetics, 93(2):91-108, 2005.

[15] O. Diekmann. Delay equations: functional-, complex-, and nonlinear analysis. Springer, 1995.

[16] O. Diekmann and S. van Gils. The center manifold for delay equations in the light of suns and stars. Singularity Theory and its Applications, pages 122-141, 1991.

[17] N. Dunford and J.T. Schwartz. Linear operators: Spectral operators, volume 7. WileyInterscience, 1988.

[18] K.J. Engel and R. Nagel. One-parameter semigroups for linear evolution equations, volume 63. Springer, 2001.

[19] G.B. Ermentrout and J.D. Cowan. Large scale spatially organized activity in neural nets. SIAM Journal on Applied Mathematics, pages 1-21, 1980.

[20] T. Faria. Normal forms for semilinear functional differential equations in banach spaces and applications. part ii. Discrete and Continuous Dynamical Systems (DCDS-A), 7(1):155-176, 2001.

[21] T. Faria, W. Huang, and J. Wu. Smoothness of center manifolds for maps and formal adjoints for semilinear fdes in general banach spaces. SIAM Journal on Mathematical Analysis, $34: 173,2002$.

[22] T. Faria and LT Magalhaes. Normal forms for retarded functional differential equations with parameters and applications to hopf bifurcation. Journal of differential equations, 122(2):181-200, 1995. 
[23] O. Faugeras, F. Grimbert, and J.-J. Slotine. Abolute stability and complete synchronization in a class of neural fields models. SIAM Journal of Applied Mathematics, 61(1):205-250, September 2008.

[24] J.K. Hale and S.M.V. Lunel. Introduction to functional differential equations. Springer Verlag, 1993.

[25] M. Haragus and G. Iooss. Local bifurcations, center manifolds, and normal forms in infinite dimensional systems. EDP Sci. Springer Verlag UTX series, 2010.

[26] H.J. Hupkes and S.M.V. Lunel. Center manifold theory for functional differential equations of mixed type. Journal of Dynamics and Differential Equations, 19(2):497-560, 2007.

[27] A. Hutt. Local excitation-lateral inhibition interaction yields oscillatory instabilities in nonlocally interacting systems involving finite propagation delays. Physics Letters A, 372:541$546,2008$.

[28] A. Hutt. Finite propagation speeds in spatially extended systems. Complex Time-Delay Systems: Theory and Applications, page 151, 2009.

[29] G. Iooss and K. Kirchgassner. Travelling waves in a chain of coupled nonlinear oscillators. Communications in Mathematical Physics, 211(2):439-464, 2000.

[30] VK Jirsa and JAS Kelso. Spatiotemporal pattern formation in neural systems with heterogeneous connection topologies. Physical Review E, 62(6):8462-8465, 2000.

[31] T. Kato. Perturbation Theory for Linear Operators. Springer, 1995.

[32] Yuri A. Kuznetsov. Elements of Applied Bifurcation Theory. Applied Mathematical Sciences. Springer, 2nd edition, 1998.

[33] V. Markounikau, C. Igel, A. Grinvald, and D. Jancke. A dynamic neural field model of mesoscopic cortical activity captured with voltage-sensitive dye imaging. PLoS Comput Biol, 6(9):e1000919, 2010.

[34] S. Nakagiri. Structural properties of functional differential equations in banach spaces. Osaka J. Math, 25:353-398, 1988.

[35] S.I. Nakagiri. Optimal control of linear retarded systems in banach spaces. Journal of mathematical analysis and applications, 120(1):169-210, 1986.

[36] D.J. Pinto, J.C. Brumberg, D.J. Simons, G.B. Ermentrout, and R. Traub. A quantitative population model of whisker barrels: re-examining the wilson-cowan equations. Journal of Computational Neuroscience, 3(3):247-264, 1996.

[37] V. A. Pliss. The reduction principle in the theory of the stability of motion. Izv. Akad. Nauk SSSR, Ser Mat., (27):1297-1324, 1964.

[38] A. Roxin, N. Brunel, and D. Hansel. Role of Delays in Shaping Spatiotemporal Dynamics of Neuronal Activity in Large Networks. Physical Review Letters, 94(23):238103, 2005.

[39] A. Roxin and E. Montbrió. How effective delays shape oscillatory dynamics in neuronal networks. Physica. D, 240(3):323-345, 2011.

[40] CC Travis and GF Webb. Existence and stability for partial functional differential equations. AMERICAN MATHEMATICAL SOCIETY, 200, 1974. 
[41] A. Vanderbauwhede and G. Iooss. Center manifold theory in infinite dimensions. Dynamics Reported PJ-Expositions in Dynamical Systems. Vol. 1, New Series. Springer-Verlag, Berlin, page $125,1992$.

[42] A. Vanderbauwhede and SA Van Gils. Center manifolds and contractions on a scale of banach spaces. Journal of Functional Analysis, 72(2):209-224, 1987.

[43] Romain Veltz. An analytical method for computing hopf bifurcation curves in neural field networks with space-dependent delays. Comptes Rendus Mathematique, 349:749-752, July 2011.

[44] Romain Veltz and Olivier Faugeras. Local/global analysis of the stationary solutions of some neural field equations. SIAM Journal on Applied Dynamical Systems, 9(3):954-998, August 2010.

[45] Romain Veltz and Olivier Faugeras. Stability of the stationary solutions of neural field equations with propagation delays. The Journal of Mathematical Neuroscience, 1(1):1, 2011.

[46] GF Webb. Functional differential equations and nonlinear semigroups in lp-spaces. Journal of Differential Equations, 20(1):71-89, 1976.

[47] J. Wu. Theory and applications of partial functional differential equations. Springer, 1996.

[48] J. Wu. Symmetric functional differential equations and neural networks with memory. Transactions of the American Mathematical Society, 350(12):4799-4838, 1998.

[49] K. Yosida. Functional Analysis. 6th ed., volume XII of Grundlehren der mathematischen Wissenschaften. Springer-Verlag, 1980. 


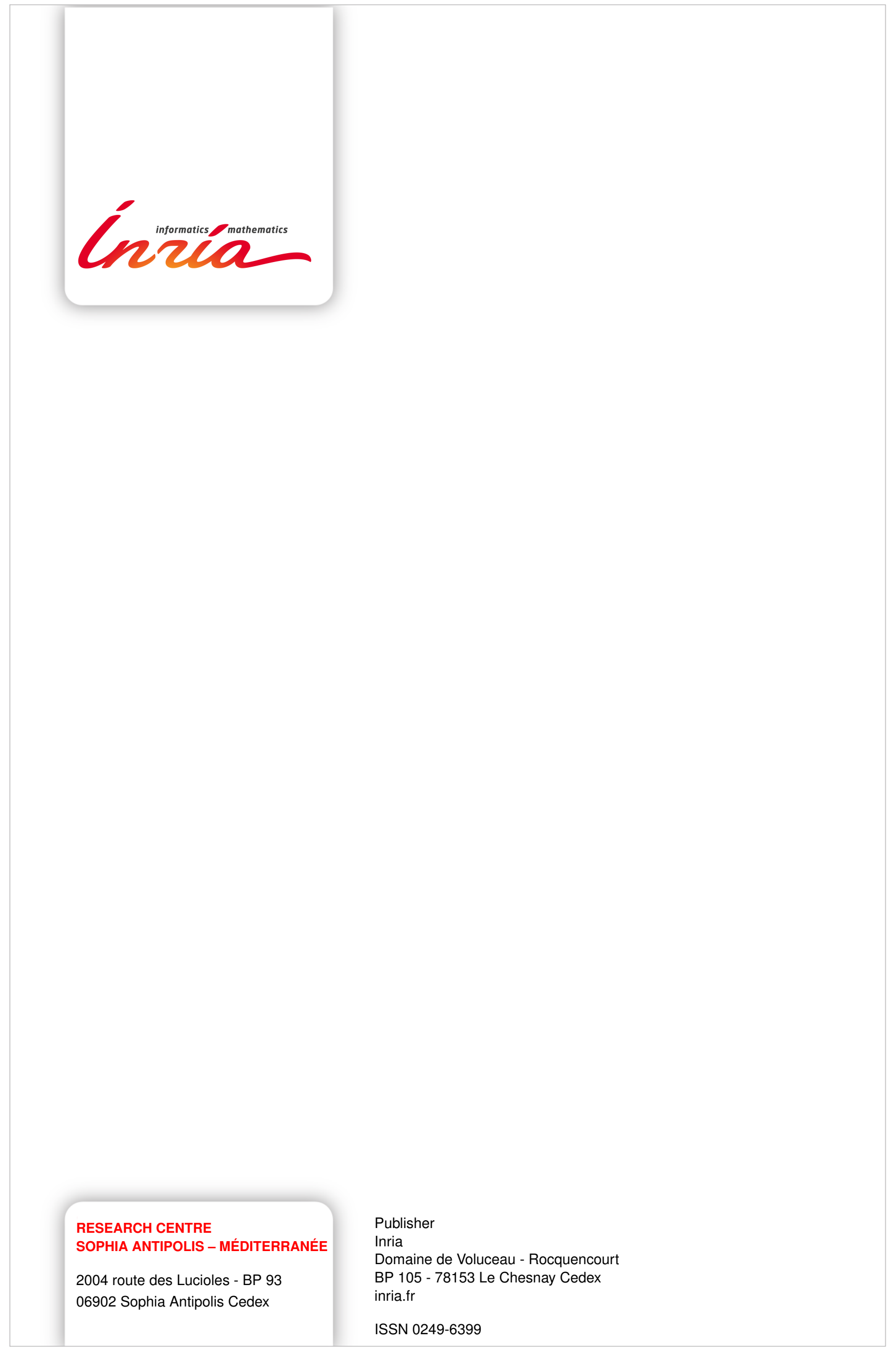

